\title{
Organocatalytic Asymmetric Cascade Reaction of 2-Hydroxyphenyl-substituted Enones and Isocyanates To \\ Construct 1,3-Benzoxazin-2-ones
}

Songsong Guo, Xiaohua Liu*, Bin Shen, Lili Lin and Xiaoming Feng

Key Laboratory of Green Chemistry \& Technology, Ministry of Education, College of Chemistry, Sichuan University, Chengdu 610064, China.

E-mail: liuxh@scu.edu.cn

Table of Contents

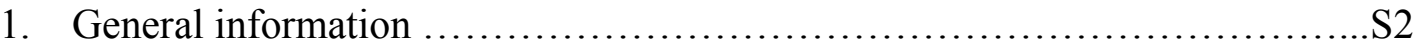

2. Typical procedure for guanidines and guanidinium salts preparation..........S2-5

3. Optimization of the reaction conditions.................................... 5

4. Typical procedure for the cascade aza-Michael reaction .....................S10

5. The analytical and spectral characterization data of the products .............S10-29

6. Gram scale experiment................................................. 29

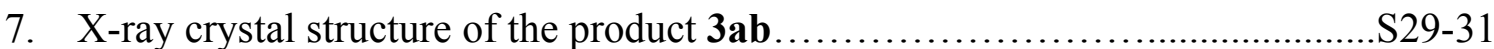

8. The mechanism study of cascade aza-Michael reaction...........................S31-32

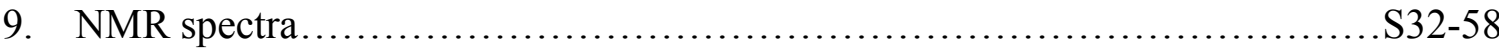

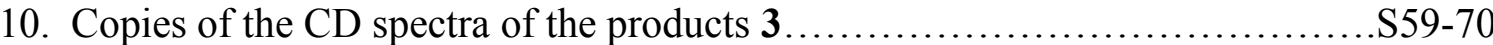

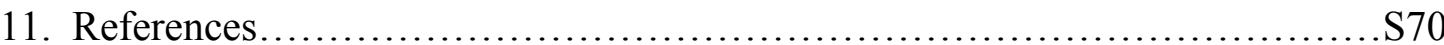




\section{General information}

${ }^{1} \mathrm{H}$ NMR spectra were recorded on commercial instruments $(400 \mathrm{MHz})$. Chemical shifts were reported in ppm from tetramethylsilane with the solvent resonance as the internal standard $\left(\mathrm{CDCl}_{3}, \delta=\right.$ 7.26). Spectra were reported as follows: chemical shift $(\delta \mathrm{ppm})$, multiplicity $(\mathrm{s}=$ singlet, $\mathrm{d}=$ doublet, $\mathrm{t}$ $=$ triplet, $\mathrm{q}=$ quartet, $\mathrm{m}=$ multiplet $)$, coupling constants $(\mathrm{Hz})$, integration and assignment. ${ }^{13} \mathrm{CNMR}$ spectra were collected on commercial instruments $(101 \mathrm{MHz})$ with complete proton decoupling. Chemical shifts are reported in ppm from the tetramethylsilane with the solvent resonance as internal standard $\left(\mathrm{CDCl}_{3}, \delta=77.0\right)$.

Enantiomeric excesses (ee) were determined by HPLC analysis using the corresponding commercial chiralpak column as stated in the experimental procedures at $20^{\circ} \mathrm{C}$.

Optical rotations were reported as follows: $[\alpha]_{\mathrm{D}}{ }^{18}(c: \mathrm{g} / 100 \mathrm{~mL}$, in solvent).

HRMS was recorded on a commercial apparatus (ESI Source).

All catalytic reactions were run in dried glassware. THF, toluene and diethyl ether $\left(\mathrm{Et}_{2} \mathrm{O}\right)$ were distilled from sodium benzophenone ketyl. $\mathrm{CH}_{2} \mathrm{Cl}_{2}$ was distilled over $\mathrm{CaH}_{2}$.

The preparation of 2-hydroxyphenyl-substituted enones ${ }^{1}$, guanidines ${ }^{2}$ and guanidinium salts $^{3}$ followed the literature.

\section{Typical procedure for guanidines and guanidinium salts preparation}
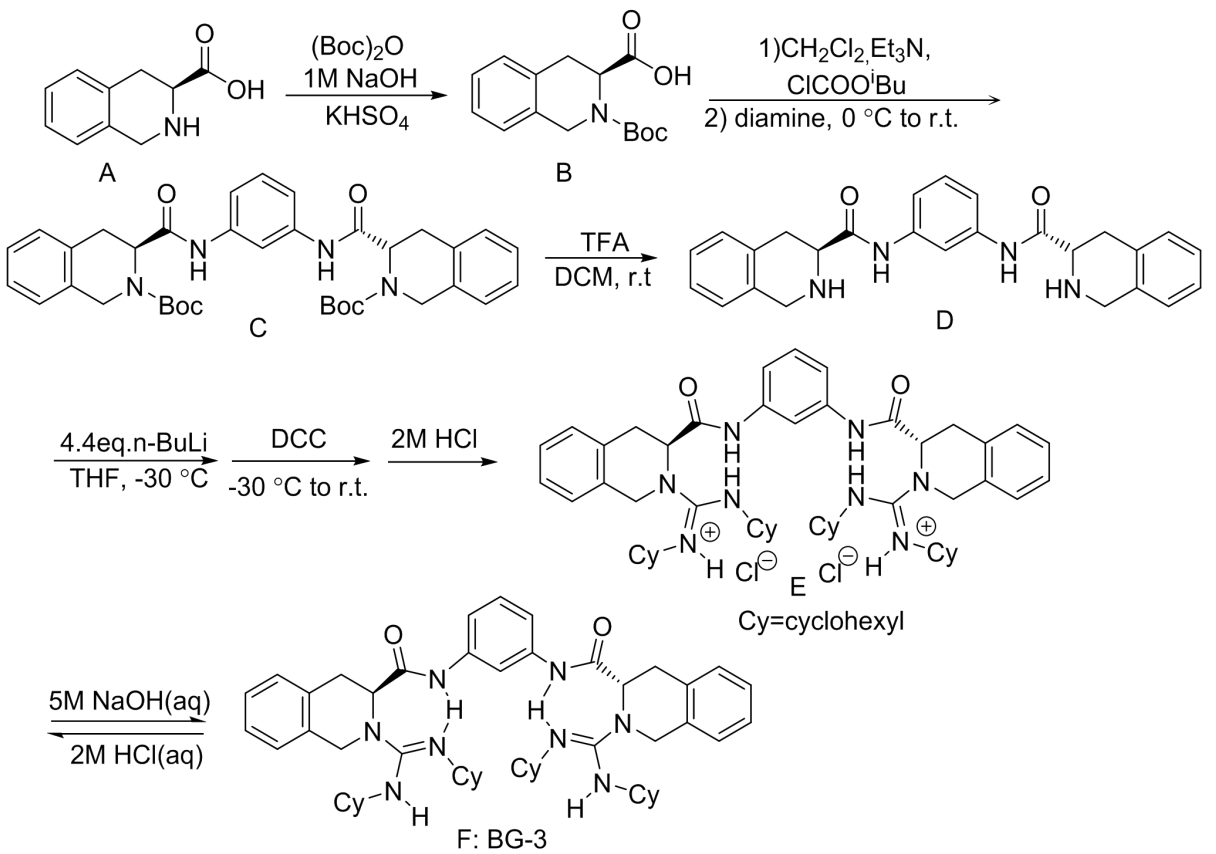
To a solution of (S)-1,2,3,4-tetrahydro-3-isoquinolinecarboxlic acid A (7.088 g, $40.0 \mathrm{mmol})$ in water-dioxane $(\mathrm{v} / \mathrm{v}=1 / 2,75 \mathrm{~mL}), \mathrm{NaOH}(1 \mathrm{M}, 40 \mathrm{~mL})$ followed by di-tert-butyl dicarbonate $(48.0$ mmol, $11 \mathrm{~mL}, 1.2$ equiv) were added at room temperature. The reaction mixture was stirred at room temperature overnight. Dioxane was evaporated in vacuo and dissolved with EtOAc. The $\mathrm{pH}$ was adjusted to 2-4 by adding aqueous $\mathrm{KHSO}_{4}$, and the product was extracted with EtOAc $(2 \times 30 \mathrm{~mL})$. The solvent was evaporated to give $\mathbf{B}$ in nearly quantitative yield (10.858 g, 98\%). It was used directly in the next step without further purification.

To a solution of $\mathbf{B}(4.274 \mathrm{~g}, 15.4 \mathrm{mmol})$ in $\mathrm{Cl}_{2} \mathrm{CH}_{2}(30 \mathrm{~mL})$ was added triethylamine $(2.1 \mathrm{~mL}, 15.0$ mmol), isobutyl carbonochloridate $(2.0 \mathrm{~mL}, 15.0 \mathrm{mmol})$ at $0{ }^{\circ} \mathrm{C}$ under stirring. After 30 minutes, benzene-1,3-diamine $(788 \mathrm{mg}, 7.3 \mathrm{mmol})$ was added. The reaction was allowed to warm to room temperature and detected by TLC. After $24 \mathrm{~h}$, the mixture was washed with sat. $\mathrm{KHSO}_{4}$, sat. $\mathrm{NaHCO}_{3}$, and brine, subsequently, then dried over anhydrous $\mathrm{Na}_{2} \mathrm{SO}_{4}$ and concentrated to give the crude product C. TFA $(15 \mathrm{~mL})$ was added to the $\mathrm{CH}_{2} \mathrm{Cl}_{2}(10 \mathrm{~mL})$ solution of the $N$-Boc protected amide $\mathbf{C}$ and stirred until the reaction finished $(1 \mathrm{~h})$. Then, $\mathrm{CH}_{2} \mathrm{Cl}_{2}(100 \mathrm{~mL})$ and $\mathrm{H}_{2} \mathrm{O}(10 \mathrm{~mL})$ was added. The $\mathrm{pH}$ value of the mixture was brought into the range of 10-12 by the addition of aq. $\mathrm{NaOH}(1 \mathrm{M})$. The aqueous phase was extracted with $\mathrm{CH}_{2} \mathrm{Cl}_{2}(3 \times 50 \mathrm{~mL})$. The combined organic phase was washed with brine, dried over anhydrous $\mathrm{Na}_{2} \mathrm{SO}_{4}$, evaporated in vacuo and recrystallized to get a kind of white solid D $(2.209 \mathrm{~g}, 71 \%)$.

$n$-BuLi (4.4 equiv, 2.4 $\mathrm{M}$ in $n$-hexane, $7.1 \mathrm{~mL}, 17.6 \mathrm{mmol}$ ) was injected into a solution of $\mathbf{D}(1.704$ $\mathrm{g}, 4.0 \mathrm{mmol})$ in THF $(70 \mathrm{~mL})$ dropwise over 30 minutes under nitrogen atmosphere at $-30{ }^{\circ} \mathrm{C}$ with well stirring. For additional 30 minutes, a solution of DCC (4.0 equiv, $3.3 \mathrm{~g}, 16.0 \mathrm{mmol}$ ) in THF was added dropwise in 20 minutes. The reaction was allowed to arm to room temperature. After $48 \mathrm{~h}$, the mixture was evaporated under reduced pressure to get rid of THF, and the $\mathrm{pH}$ value of the mixture was brought into the range of $0-1$ by the addition of $2 \mathrm{M} \mathrm{HCl}$. The aqueous phase was extracted with $\mathrm{CH}_{2} \mathrm{Cl}_{2}(5 \times 30 \mathrm{~mL})$. The combined organic phase was washed with brine, dried over anhydrous $\mathrm{MgSO}_{4}$ and evaporated in vacuum and purified by column chromatography to give the product $\mathbf{E}$. The white solid $\mathbf{E}$ in $\mathrm{CH}_{2} \mathrm{Cl}_{2}(16 \mathrm{~mL})$ was added $\mathrm{NaOH}(5 \mathrm{M}, 15 \mathrm{~mL})$ and stirred until the basification was finished (10 minutes). The $\mathrm{pH}$ value of the mixture was kept in the range of 11-12. The aqueous phase was extracted with $\mathrm{CH}_{2} \mathrm{Cl}_{2}(5 \times 20 \mathrm{~mL})$. The combined organic phase was washed with brine, dried over anhydrous $\mathrm{Na}_{2} \mathrm{SO}_{4}$ and evaporated in vacuum. Finally a white solid $\mathbf{F}$ was obtained. Then it was dissolved in $\mathrm{CH}_{2} \mathrm{Cl}_{2}$ and filtration through celite to remove the silicone gel, concentrate to get a white solid F (0.919 g, 27\% yield). For other catalysts, the synthesis method and ${ }^{1} \mathrm{H}$ NMR and ${ }^{13} \mathrm{C}$ NMR spectra could be found the literature. ${ }^{2}$

The synthesis of the compound $\mathbf{B G}-\mathbf{3} \cdot 1.3 \mathrm{HBAr}^{\mathrm{F}}$ from compound $\mathbf{E}$ was as follows: To a solution of the white solid $\mathbf{E}(91.1 \mathrm{mg}, 0.1 \mathrm{mmol})$ which dissolved in $\mathrm{CH}_{2} \mathrm{Cl}_{2}(16 \mathrm{~mL})$ was added $\mathrm{NaBAr}_{4}$ $\left(\mathrm{NaBAr}_{4}{ }_{4}=\mathrm{NaB}\left[3,5-\left(\mathrm{F}_{3} \mathrm{C}\right)_{2} \mathrm{C}_{6} \mathrm{H}_{3}\right]_{4}\right)(2.0$ equiv, $177.2 \mathrm{mg}, 0.2 \mathrm{mmol})$ at room temperature under 
stirring for $5 \mathrm{~h}$. Then, the mixture was filtrated through celite to remove $\mathrm{NaCl}$, and concentrated to get a white solid G (246.0 mg, 93\%).

To a solution of white solid $\mathbf{F}(29.4 \mathrm{mg}, 0.035 \mathrm{mmol})$ in $\mathrm{Cl}_{2} \mathrm{CH}_{2}(20 \mathrm{~mL})$ was added white solid $\mathbf{G}$ (166.4 $\mathrm{mg}, 0.065 \mathrm{mmol}$ ) for 30 minutes, and evaporated in vacuo to obtain white solid BG-3 $1.3 \mathrm{HBAr}_{4}{ }_{4}$ in quantitative yield.

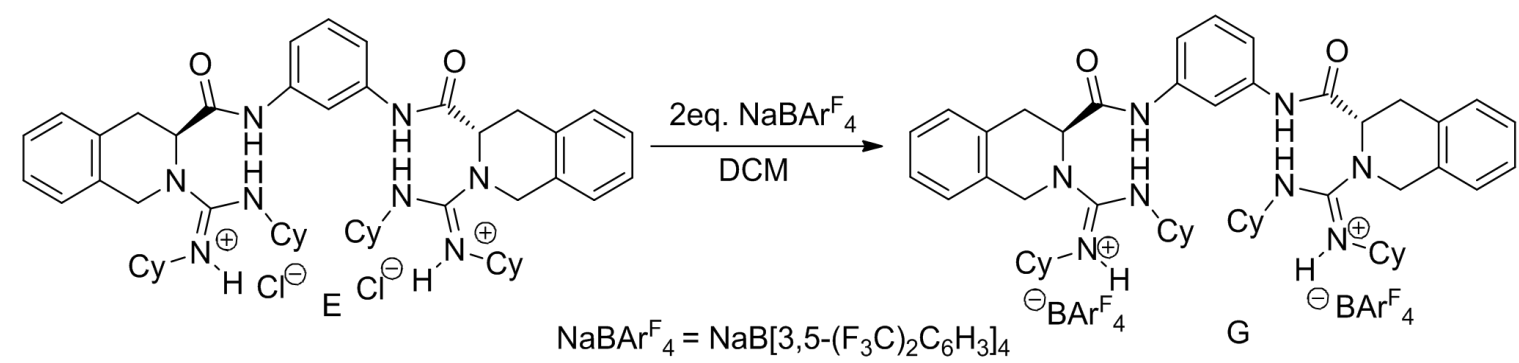
$\underset{\mathrm{DCM}}{\stackrel{\mathrm{F}}{\longrightarrow}} \mathrm{BG}-3 \cdot 1.3 \mathrm{HBAr}_{4}$<smiles>CNC1N(Cl)CN(c2cccc(NC(=O)C3Cc4ccccc4CN3C(=O)N(C)C3CCCCC3)c2)[C@@H]2Cc3ccccc3CN12</smiles>

BG-3 (F)

${ }^{1} \mathrm{H}$ NMR $\left(400 \mathrm{MHz}, \mathrm{CDCl}_{3}\right) \delta 12.12(\mathrm{~s}, 2 \mathrm{H})$, $7.89(\mathrm{~s}, 1 \mathrm{H}), 7.21(\mathrm{~d}, J=7.2 \mathrm{~Hz}, 4 \mathrm{H}), 7.11(\mathrm{dt}, J$ $=15.4,7.2 \mathrm{~Hz}, 5 \mathrm{H}), 6.95(\mathrm{~d}, J=7.6 \mathrm{~Hz}, 2 \mathrm{H})$, $4.85(\mathrm{~d}, J=6.0 \mathrm{~Hz}, 2 \mathrm{H}), 4.33(\mathrm{~d}, J=16.4 \mathrm{~Hz}$, 2H), 4.08 (dd, $J=28.8,12.0 \mathrm{~Hz}, 3 \mathrm{H}), 3.44$ (dd, $J$ $=20.4,13.2 \mathrm{~Hz}, 5 \mathrm{H}), 3.09-2.84(\mathrm{~m}, 7 \mathrm{H}), 2.00-$

$1.82(\mathrm{~m}, 5 \mathrm{H}), 1.62$ (qdd, $J=33.2,28.0,12.4 \mathrm{~Hz}, 30 \mathrm{H}), 1.38-0.85$ (m, 24H).

${ }^{13} \mathrm{C}$ NMR $\left(101 \mathrm{MHz}, \mathrm{CDCl}_{3}\right) \delta=170.6,155.9,139.7,134.5,132.6,129.0,126.5,125.7,125.3$, $114.2,56.1,53.6,47.4,35.8,35.4,34.9,34.0,33.6,29.3,25.6,25.6,25.5,25.3,25.0,24.8$.

HRMS (ESI) calcd for $[\mathbf{M}+\mathbf{H}]^{+} \mathbf{C}_{52} \mathbf{H}_{71} \mathbf{N}_{\mathbf{8}} \mathbf{O}_{2}{ }^{+}, \mathrm{m} / \mathrm{z}: 839.5700$, observed: 839.5701 .

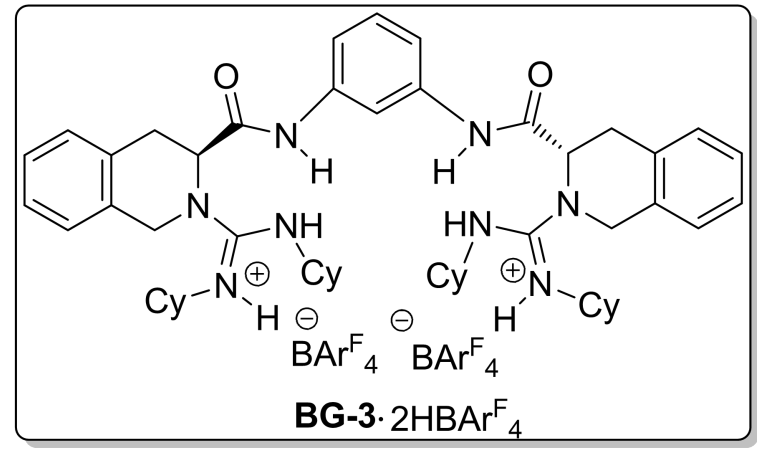

BG-3 $2 \mathrm{HBAr}_{4}(\mathbf{G})$

${ }^{1} \mathrm{H}$ NMR $\left(400 \mathrm{MHz}, \mathrm{CDCl}_{3}\right) \delta 7.71(\mathrm{~s}, 16 \mathrm{H})$, 7.48 (s, 8H), 7.34 (s, 1H), 7.24 (ddd, $J=27.4$, 13.5, $6.5 \mathrm{~Hz}, 10 \mathrm{H}), 7.04$ (t, $J=8.0 \mathrm{~Hz}, 3 \mathrm{H}), 6.77$ (d, $J=8.0 \mathrm{~Hz}, 4 \mathrm{H}), 4.55(\mathrm{~s}, 2 \mathrm{H}), 4.41(\mathrm{dd}, J=$ $39.2,15.6 \mathrm{~Hz}, 4 \mathrm{H}), 3.34(\mathrm{~d}, J=11.6 \mathrm{~Hz}, 6 \mathrm{H})$, $3.11(\mathrm{~d}, J=17.2 \mathrm{~Hz}, 2 \mathrm{H}), 2.10(\mathrm{~s}, 1 \mathrm{H}), 2.02-$ $1.82(\mathrm{~m}, 8 \mathrm{H}), 1.80-1.51(\mathrm{~m}, 15 \mathrm{H}), 1.44-1.03(\mathrm{~m}, 21 \mathrm{H})$.

${ }^{13} \mathrm{C}$ NMR $\left(101 \mathrm{MHz}, \mathrm{CDCl}_{3}\right) \delta=168.4,162.4,161.9,161.5,161.0,158.6,135.9,134.8,130.0$, $129.5,129.4,129.0,129.0,128.9,128.7,128.6,128.4,126.7,125.9,123.2,120.5,118.2,117.5,112.6$, 
58.6, 55.0, 48.2, 33.9, 32.8, 30.1, 24.4, 24.2, 24.1.

HRMS (ESI) calcd for $[\mathbf{M}+\mathbf{N a}]^{+} \mathbf{C}_{\mathbf{1 1 6}} \mathbf{H}_{\mathbf{9} 6} \mathbf{B}_{\mathbf{2}} \mathbf{F}_{48} \mathbf{N}_{\mathbf{8}} \mathbf{O}_{2} \mathbf{N a}^{+}$, m/z: 2589.6973 , observed: 2589.6965

\section{Optimization of the reaction conditions}

Table 1: Screening of guanidines and guanidinium salts ${ }^{[a]}$
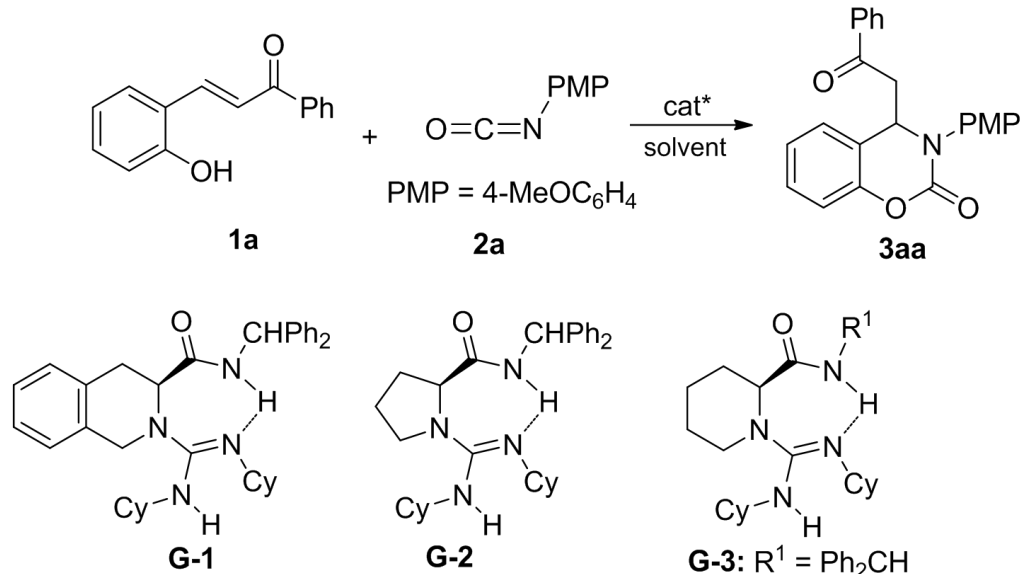

(Cy $=$ Cyclohexyl)

G-3: $\mathrm{R}^{1}=\mathrm{Ph}_{2} \mathrm{CH}$

G-4: $\mathrm{R}^{1}=2,6-\mathrm{Me}_{2} \mathrm{C}_{6} \mathrm{H}_{3}$ G-5: $\mathrm{R}^{1}=2,6-\mathrm{iPr}_{2} \mathrm{C}_{6} \mathrm{H}_{3}$

G-6: $\mathrm{R}^{1}=2,4,6-i \mathrm{Pr}_{3} \mathrm{C}_{6} \mathrm{H}_{2}$<smiles>[R]NC(=O)C1C2CCCC2CN1/C(=N/Cl)NC(=O)NC</smiles>

G-7: $\mathrm{R}^{2}=\mathrm{Ph}_{2} \mathrm{CH}$

G-8: $R^{2}=1$-adamantyl

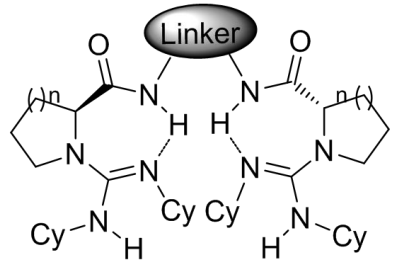<smiles>CC(Cc1ccccc1)C(C)(C)c1ccccc1</smiles>
BG-4: $n=1$, Linker

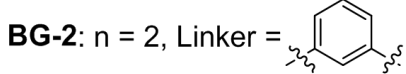<smiles>CCNC(=O)C1Cc2ccccc2CN1C(=NC1CCCCC1)NC1CCCCC1</smiles><smiles></smiles>

BG-3

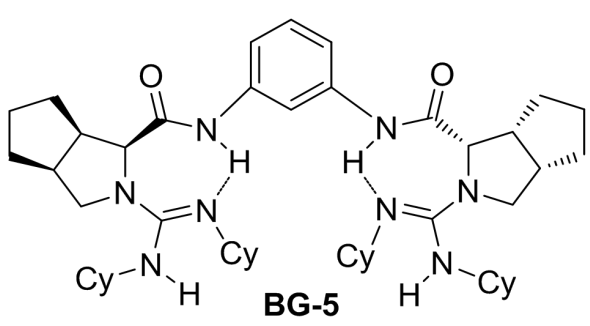

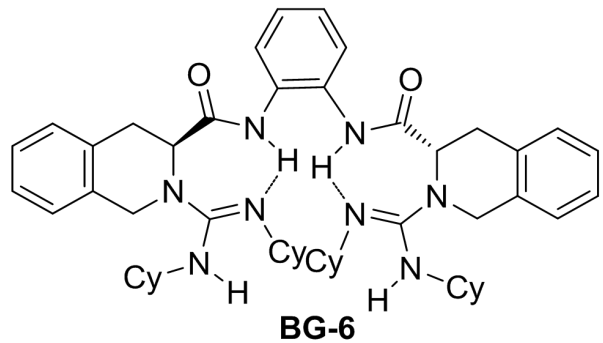

\begin{tabular}{ccccc}
\hline entry & cat. & t $(\mathrm{h})$ & yield $(\%)^{[\mathrm{b}]}$ & ee $(\%)^{[\mathrm{c}]}$ \\
\hline 1 & G-1 & 20 & $<6$ & 11 \\
2 & G-2 & 20 & 91 & 0 \\
\hline
\end{tabular}




\begin{tabular}{|c|c|c|c|c|}
\hline 3 & G-3 & 20 & 45 & 24 \\
\hline 4 & G-4 & 24 & 44 & 29 \\
\hline 5 & G-5 & 22 & 13 & 20 \\
\hline 6 & G-6 & 26 & 32 & 19 \\
\hline 7 & G-7 & 30 & 52 & -7 \\
\hline 8 & G-8 & 14 & 80 & 0 \\
\hline 9 & BG-1 & 20 & 88 & 0 \\
\hline 10 & BG-2 & 20 & 98 & 9 \\
\hline 11 & BG-3 & 20 & 81 & 25 \\
\hline 12 & BG-4 & 34 & 65 & 0 \\
\hline 13 & BG-5 & 26 & 39 & 19 \\
\hline 14 & BG-6 & 18 & 47 & 3 \\
\hline 15 & BG-2 $\cdot \mathrm{HBAr}^{\mathrm{F}}$ & 12 & 16 & -10 \\
\hline 16 & BG-3 $\cdot \mathrm{HBAr}^{\mathrm{F}}$ & 24 & 82 & 58 \\
\hline
\end{tabular}

[a] Unless otherwise noted, the reactions were carried out with $10 \mathrm{~mol} \%$ of catalyst, $0.10 \mathrm{mmol} \mathbf{1 a}$ and $0.12 \mathrm{mmol} 2 \mathrm{a}$ in $1.0 \mathrm{~mL} \mathrm{CH} \mathrm{Cl}_{2}$ at $30{ }^{\circ} \mathrm{C}$. [b] Isolated yield. [c] Determined by HPLC analysis (Chiralcel OD-H).

Table 2: Screening of the solvents ${ }^{[a]}$

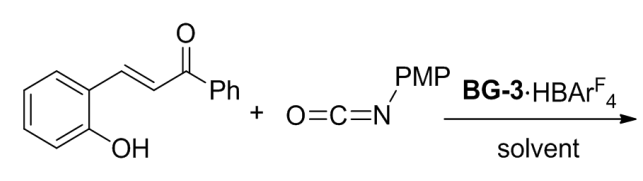

1a<smiles>NP(F)(F)(F)N1C(=O)Oc2ccccc2C1CC(=O)c1ccccc1</smiles>

3aa

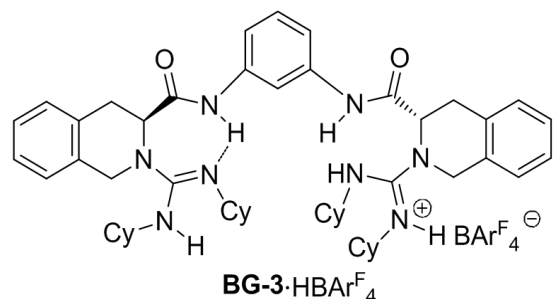

BG-3. $\mathrm{HBArF}_{4}$ 


\begin{tabular}{ccccc}
\hline 1 & Toluene & 20 & 90 & 65 \\
2 & $\mathrm{THF}$ & 20 & trace & $/$ \\
3 & $\mathrm{CH}_{2} \mathrm{Cl}_{2}$ & 20 & 80 & 70 \\
4 & $\mathrm{Et} 2 \mathrm{O}$ & 20 & 99 & 72 \\
5 & MTBE & 19 & 99 & 69 \\
6 & 1,4 -dioxane & 19 & 54 & 63 \\
7 & n-Butyl ether & 19 & 71 & 37 \\
\hline
\end{tabular}

[a] Unless otherwise noted, the reactions were carried out with $10 \mathrm{~mol} \%$ of catalyst $\mathbf{B G}-3 \cdot \mathrm{HBAr}_{4}$, $0.10 \mathrm{mmol} 1 \mathrm{a}$ and $0.12 \mathrm{mmol} \mathrm{2a}$ in $1.0 \mathrm{~mL}$ solvent at $30^{\circ} \mathrm{C}$. [b] Isolated yield. [c] Determined by HPLC analysis (Chiralcel OD-H).

Table 3: Screening of the amount of solvent ${ }^{[a]}$
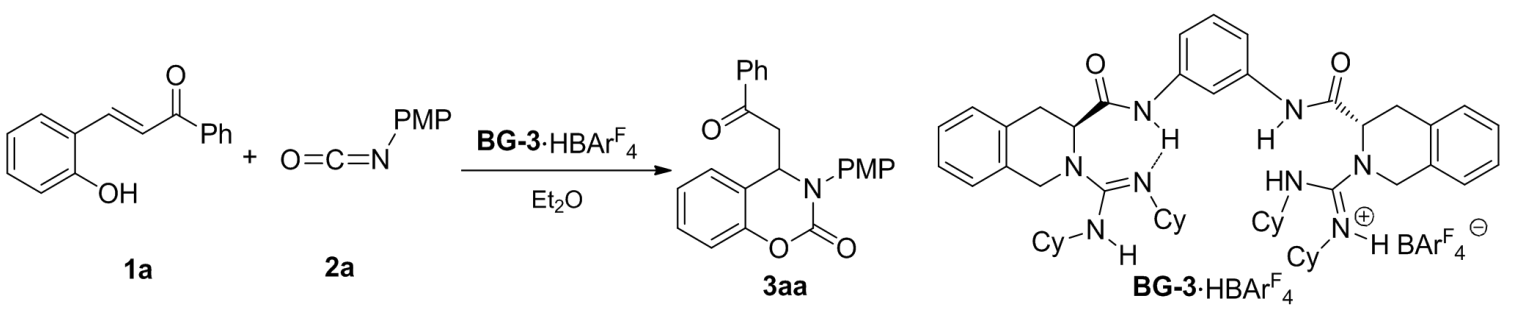

\begin{tabular}{ccccc}
\hline entry & amount of solvent $(\mathrm{mL})$ & $\mathrm{t}(\mathrm{h})$ & ${\text { yield }(\%)^{[\mathrm{b}]}}$ & ee (\%) \\
\hline 1 & 2.0 & 18 & 91 & 70 \\
2 & 1.5 & 18 & 94 & 72 \\
3 & 1.2 & 18 & $>99$ & 73 \\
4 & 1.0 & 18 & $>99$ & 70 \\
5 & 0.6 & 18 & $>99$ & 70 \\
\hline
\end{tabular}

[a] Unless otherwise noted, the reactions were carried out with $10 \mathrm{~mol} \%$ of catalyst $\mathbf{B G}-\mathbf{3} \cdot \mathrm{HBAr}^{\mathrm{F}}$, $0.05 \mathrm{mmol} \mathbf{1 a}$ and $0.06 \mathrm{mmol} \mathbf{2 a}$ in $\mathrm{Et}_{2} \mathrm{O}$ at $30^{\circ} \mathrm{C}$. [b] Isolated yield. [c] Determined by HPLC analysis (Chiralcel OD-H). 
Table 4: Screening of the reaction temperature ${ }^{[a]}$

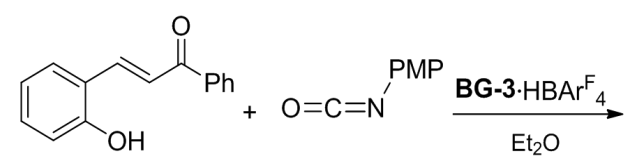

$1 \mathrm{a}$

2a<smiles>NP(=O)(c1ccccc1)N1C(=O)Oc2ccccc2C1CC(=O)c1ccccc1</smiles>

3aa

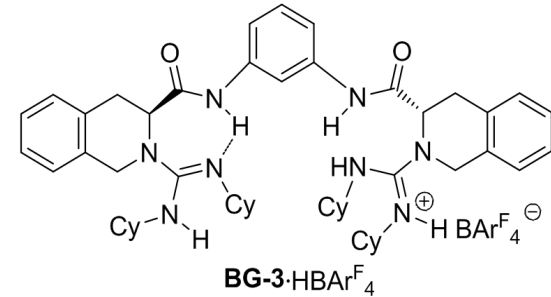

yield $(\%)^{[b]}$

ee $(\%)^{[c]}$

\begin{tabular}{ccccc}
\hline 1 & 35 & 18 & 80 & 70 \\
2 & 30 & 18 & 82 & 75 \\
3 & 25 & 18 & 72 & 72 \\
4 & 0 & 38 & 96 & 80 \\
5 & -30 & 46 & 24 & 85 \\
6 & -40 & 49 & - & - \\
$7^{[\mathrm{d}]}$ & 0 & 38 & 98 & 84
\end{tabular}

[a] Unless otherwise noted, the reactions were carried out with $10 \mathrm{~mol} \%$ of catalyst $\mathbf{B G}-\mathbf{3} \cdot \mathrm{HBAr}^{\mathrm{F}}$, $0.05 \mathrm{mmol} \mathbf{1 a}$ and $0.06 \mathrm{mmol} \mathbf{2 a}$ in $\mathrm{Et}_{2} \mathrm{O}(1.2 \mathrm{~mL})$. [b] Isolated yield. [c] Determined by HPLC analysis (Chiralcel OD-H). [d] The reactions were carried out with $5 \mathrm{~mol} \%$ catalyst BG-3 $\cdot \mathrm{HBAr}^{\mathrm{F}} 4$ at $0{ }^{\circ} \mathrm{C}$ in $1.2 \mathrm{~mL} \mathrm{Et}_{2} \mathrm{O}$.

Table 5: Screening of guanidium salts ${ }^{[a]}$

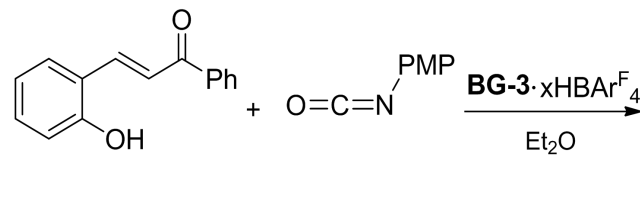

$1 \mathrm{a}$<smiles>O=C(CC1c2ccccc2OC(=O)N1[18OH])c1ccccc1</smiles>

3aa

\begin{tabular}{rcccc}
\hline Entry & BG-3 $\cdot \mathrm{xHBAr}_{4}^{\mathrm{F}}$ & $\mathrm{t}(\mathrm{h})$ & ${\text { Yield }(\%)^{[\mathrm{b}]}}$ & $\mathrm{Ee}(\%)^{[\mathrm{c}]}$ \\
\hline 1 & 2 & 46 & - & - \\
2 & 1.5 & 46 & 11 & 84 \\
\hline
\end{tabular}




\begin{tabular}{ccccc}
\hline 3 & 1.3 & 46 & 24 & 85 \\
4 & 1 & 46 & 21 & 80 \\
5 & 0.5 & 46 & 11 & 74 \\
6 & 0 & 46 & 7 & 59 \\
$7^{[\mathrm{d}]}$ & 1.3 & 39 & 99 & 89 \\
\hline
\end{tabular}

[A] Unless otherwise noted, the reactions were carried out with $5 \mathrm{~mol} \%$ catalyst, $0.05 \mathrm{mmol} \mathbf{1 a}$ and $0.06 \mathrm{mmol} 2 \mathrm{a}$ in $1.2 \mathrm{~mL} \mathrm{Et} 2 \mathrm{O}$ at $-30{ }^{\circ} \mathrm{C}$ for 46 hours. [b] Isolated yield. [c] Determined by HPLC analysis (Chiralcel ODH). [d] The reactions were carried out at $0{ }^{\circ} \mathrm{C}$ and $1.5 \mathrm{ml} \mathrm{Et}_{2} \mathrm{O}$ for $39 \mathrm{~h}$.

Table 6: Screening the protective group of isocyanate $\mathbf{2}$

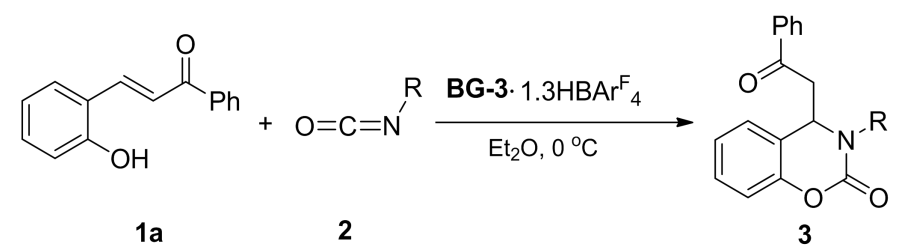

\begin{tabular}{|c|c|c|c|c|}
\hline entry & $\mathrm{R}$ & $\mathrm{t}(\mathrm{h})$ & yield $(\%)^{[b]}$ & ee $(\%)^{[c]}$ \\
\hline 1 & PMP & 30 & 99 & 89 \\
\hline 2 & $4-\mathrm{FC}_{6} \mathrm{H}_{4}$ & 46 & 99 & 93 \\
\hline 3 & $4-\mathrm{ClC}_{6} \mathrm{H}_{4}$ & 46 & 36 & 90 \\
\hline 4 & $4-\mathrm{BrC}_{6} \mathrm{H}_{4}$ & 46 & 28 & 93 \\
\hline 5 & 4- $\mathrm{MeC}_{6} \mathrm{H}_{4}$ & 59 & 95 & 73 \\
\hline 6 & $t-\mathrm{Bu}$ & 48 & I & I \\
\hline $7^{[\mathrm{d}]}$ & $4-\mathrm{FC}_{6} \mathrm{H}_{4}$ & 52 & 99 & 93 \\
\hline $8^{[\mathrm{e}]}$ & $4-\mathrm{FC}_{6} \mathrm{H}_{4}$ & 28 & trace & 93 \\
\hline
\end{tabular}

[a] Unless otherwise noted, the reactions were carried out with $5 \mathrm{~mol} \%$ of catalyst, $0.05 \mathrm{mmol} \mathrm{1a}$ and $0.75 \mathrm{mmol} 2$ in $1.5 \mathrm{~mL} \mathrm{Et}_{2} \mathrm{O}$ at $0{ }^{\circ} \mathrm{C}$. [b] Isolated yield. [c] Determined by HPLC analysis (Chiralcel OD-H). [d] At $-10{ }^{\circ} \mathrm{C}$. [e] At $-20^{\circ} \mathrm{C}$. 


\section{Typical procedure for the cascade aza-Michael reaction}

In a test tube with a magnetic stirring bar, bisguanidinium salt BG-3 $1.3 \operatorname{HBAr}_{4}{ }_{4}(5 \mathrm{~mol} \%)$ and 2-hydroxyphenyl-substituted enones $\mathbf{1 a}(0.05 \mathrm{mmol})$ in ether $(1.5 \mathrm{~mL})$ were stirred at $30^{\circ} \mathrm{C}$ for $30 \mathrm{~min}$, Then the test tube was transformed to $0{ }^{\circ} \mathrm{C}$ and the isocyanate $2(0.075 \mathrm{mmol})$ was added in one-portion. The mixture was stirred at $0{ }^{\circ} \mathrm{C}$ and detected by TLC. After completion, flash column chromatography provided the desired product (pet/EtOAc $=3 / 1$ as eluent). Then the product was directed for HPLC and NMR analysis.

\section{The analytical and spectral characterization data of the product}

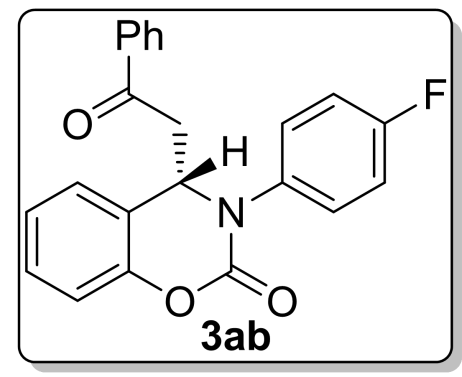

(S)-3-(4-fluorophenyl)-4-(2-oxo-2-phenylethyl)-3,4-dihydro-2H-benzo [e][1,3] oxazin-2-one (3ab).

White solid, $46 \mathrm{~h}, 17.9 \mathrm{mg}, 99 \%$ yield, 93\% ee, mp $158-160{ }^{\circ} \mathrm{C}$; $[\alpha]_{\mathrm{D}}{ }^{18}=2.14\left(c: 0.1, \mathrm{CH}_{2} \mathrm{Cl}_{2}\right)$; Determined by HPLC analysis [Daicel chiralcel OD-H, $n$-hexane $/ i$-PrOH $=70 / 30,1.0 \mathrm{~mL} / \mathrm{min}, \lambda=254 \mathrm{~nm}, \mathrm{t}$ $($ minor $)=9.84 \mathrm{~min}, \mathrm{t}($ major $)=11.65 \mathrm{~min}]$;

${ }^{1} \mathrm{H}$ NMR $\left(400 \mathrm{MHz}, \mathrm{CDCl}_{3}\right) \delta=7.82-7.74(\mathrm{~m}, 2 \mathrm{H}), 7.54(\mathrm{t}, J=$ $7.2 \mathrm{~Hz}, 1 \mathrm{H}), 7.45-7.35(\mathrm{~m}, 4 \mathrm{H}), 7.35-7.27(\mathrm{~m}, 2 \mathrm{H}), 7.18-7.05(\mathrm{~m}, 4 \mathrm{H}), 5.51$ (dd, J = 8.0, 4.0 Hz, $1 \mathrm{H}), 3.53(\mathrm{qd}, J=17.2,6.0 \mathrm{~Hz}, 2 \mathrm{H})$.

${ }^{13} \mathrm{C} \mathrm{NMR}\left(101 \mathrm{MHz}, \mathrm{CDCl}_{3}\right) \delta=196.2,162.8,160.4,150.6,149.8,136.2,136.1(\mathrm{~d}, J=2.0 \mathrm{~Hz})$, $133.8,129.5,129.1(\mathrm{~d}, J=4.0 \mathrm{~Hz}) 129.0,128.8,128.0,126.2,124.6,122.6,116.7(\mathrm{~d}, J=2.0 \mathrm{~Hz})$, $116.4,58.3,44.0$.

HRMS (ESI)caled for (M+Na) ${ }^{+} \mathbf{C}_{22} \mathbf{H}_{16} \mathbf{F N O}_{3} \mathbf{N a}^{+}, \mathrm{m} / \mathrm{z}: 384.1012$, observed: 384.1012 .

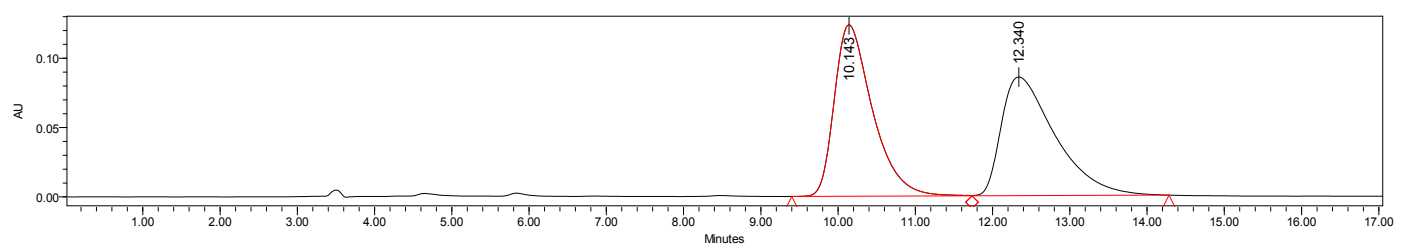

\begin{tabular}{|l|l|l|l|}
\hline & Retention Time & Area & $\%$ Area \\
\hline 1 & 10.143 & 4241427 & 50.56 \\
\hline 2 & 12.340 & 4147174 & 49.44 \\
\hline
\end{tabular}




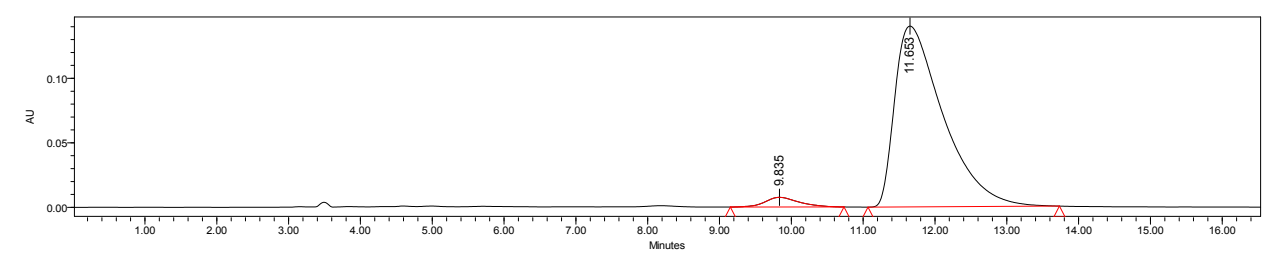

\begin{tabular}{|c|c|c|c|}
\hline & Retention Time & Area & $\%$ Area \\
\hline 1 & 9.835 & 247584 & 3.63 \\
\hline 2 & 11.653 & 6567785 & 96.37 \\
\hline
\end{tabular}

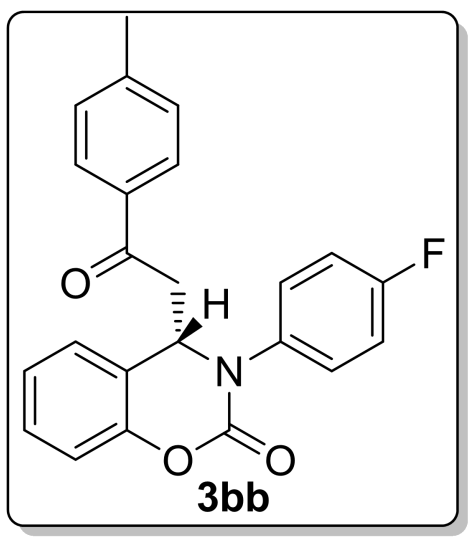

(S)-3-(4-fluorophenyl)-4-(2-oxo-2-(p-tolyl)ethyl)-3,4-dihydro-2H-b enzo[e][1,3]oxazin-2-one (3bb).

White solid, $96 \mathrm{~h}, 17.4 \mathrm{mg}, 93 \%$ yield, $93 \%$ ee, mp $84-86{ }^{\circ} \mathrm{C}$; $[\alpha]_{\mathrm{D}}{ }^{18}=1.53\left(c: 0.03, \mathrm{CH}_{2} \mathrm{Cl}_{2}\right)$; Determined by HPLC analysis [Daicel Chiralpak ODH, $n$-hexane $/ i-\mathrm{PrOH}=80 / 20,1.0 \mathrm{~mL} / \mathrm{min}, \lambda=$ $254 \mathrm{~nm}, \mathrm{t}($ minor $)=12.31 \mathrm{~min}, \mathrm{t}($ major $)=13.58 \mathrm{~min}]$.$] ;$

${ }^{1} \mathrm{H}$ NMR $\left(400 \mathrm{MHz}, \mathrm{CDCl}_{3}\right) \delta 7.68(\mathrm{~d}, J=8.4 \mathrm{~Hz}, 2 \mathrm{H}), 7.68(\mathrm{~d}$, $J=8.4 \mathrm{~Hz}, 2 \mathrm{H}), 7.43-7.36(\mathrm{~m}, 2 \mathrm{H}), 7.64-7.26(\mathrm{~m}, 6 \mathrm{H}), 7.30(\mathrm{dd}$, $J=12.4,4.4 \mathrm{~Hz}, 2 \mathrm{H}), 7.20(\mathrm{~d}, J=8.0 \mathrm{~Hz}, 2 \mathrm{H}), 7.13(\mathrm{ddd}, J=16.4$, $15.2,5.0 \mathrm{~Hz}, 6 \mathrm{H}), 7.17-7.06(\mathrm{~m}, 4 \mathrm{H}), 5.50$ (dd, $J=8.0,4.0 \mathrm{~Hz}, 1 \mathrm{H}), 5.50$ (dd, $J=8.0,4.0 \mathrm{~Hz}, 1 \mathrm{H}$ ), 3.49 (qd, $J=16.8,6.0 \mathrm{~Hz}, 2 \mathrm{H}), 3.49$ (qd, $J=16.8,6.0 \mathrm{~Hz}, 2 \mathrm{H}), 2.41$ (d, $J=22.4 \mathrm{~Hz}, 3 \mathrm{H}), 2.38(\mathrm{~s}, 3 \mathrm{H})$.

${ }^{13} \mathrm{C} \mathrm{NMR}\left(101 \mathrm{MHz}, \mathrm{CDCl}_{3}\right) \delta=195.7,162.8,160.4,150.6,149.8,144.8,136.1,133.8,129.4$, 129.1(d, $J=4.5 \mathrm{~Hz}), 128.1,126.2,124.6,122.7,116.6(\mathrm{~d}, J=1.5 \mathrm{~Hz}), 116.4,58.3,43.9,21.7$.

HRMS (ESI) calcd for $[\mathbf{M}+\mathbf{N a}]^{+} \mathbf{C}_{\mathbf{2} 3} \mathbf{H}_{\mathbf{1 8}} \mathbf{F} \mathbf{N O}_{3} \mathbf{N a}^{+}, \mathrm{m} / \mathrm{z}$ : 398.1168, observed: 398.1165.

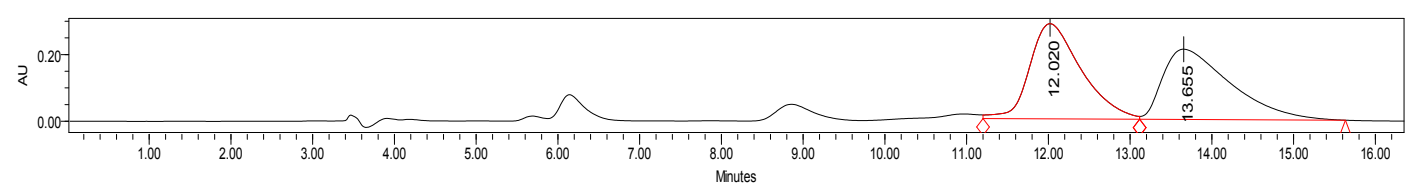

\begin{tabular}{|l|c|c|c|}
\hline & Retention Time & Area & $\%$ Area \\
\hline 1 & 12.020 & 12556903 & 50.96 \\
\hline 2 & 13.655 & 12084555 & 49.04 \\
\hline
\end{tabular}




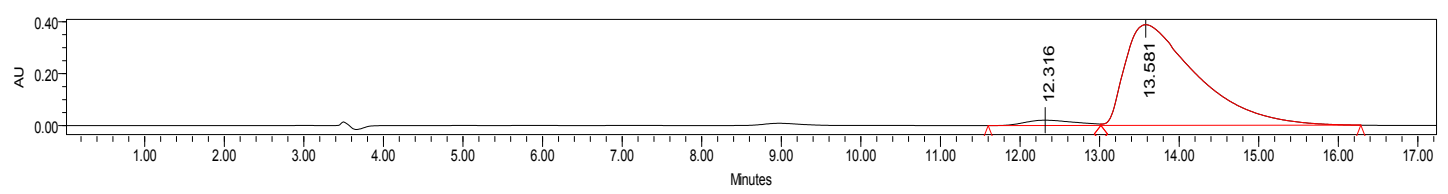

\begin{tabular}{|c|c|c|c|}
\hline & Retention Time & Area & $\%$ Area \\
\hline 1 & 12.316 & 908053 & 3.61 \\
\hline 2 & 13.581 & 24248839 & 96.39 \\
\hline
\end{tabular}

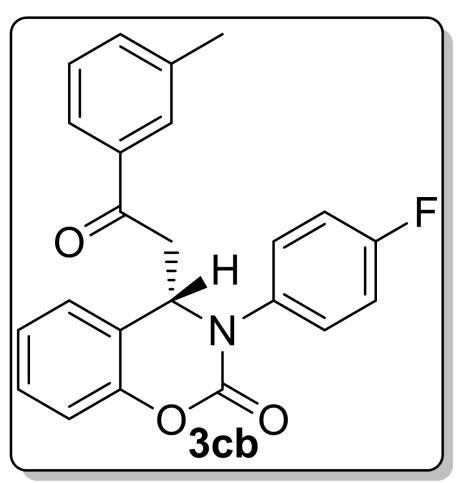

(S)-3-(4-fluorophenyl)-4-(2-oxo-2-(m-tolyl)ethyl)-3,4-dihydro-2H-ben zo[e][1,3]oxazin-2-one (3cb).

White solid, $98 \mathrm{~h}, 13.6 \mathrm{mg}, 73 \%$ yield, 92\% ee, mp 74-76 ${ }^{\circ} \mathrm{C}$; $[\alpha]_{\mathrm{D}}{ }^{18}=1.37\left(c: 0.6, \mathrm{CH}_{2} \mathrm{Cl}_{2}\right)$; Determined by HPLC analysis [Daicel Chiralpak ODH, $n$-hexane $/ i-\mathrm{PrOH}=80 / 20,1.0 \mathrm{~mL} / \mathrm{min}, \lambda=254 \mathrm{~nm}, \mathrm{t}$ $($ minor $)=11.49 \mathrm{~min}, \mathrm{t}($ major $)=12.84 \mathrm{~min}]$.$] ;$

${ }^{1} \mathrm{H}$ NMR $\left(400 \mathrm{MHz}, \mathrm{CDCl}_{3}\right) \delta=7.57(\mathrm{~d}, J=7.6 \mathrm{~Hz}, 2 \mathrm{H}), 7.43-7.24$ $(\mathrm{m}, 7 \mathrm{H}), 7.11(\mathrm{dd}, J=16.8,8.0 \mathrm{~Hz}, 4 \mathrm{H}), 5.50(\mathrm{dd}, J=8.0,4.0 \mathrm{~Hz}, 1 \mathrm{H})$, $3.51(\mathrm{qd}, J=17.2,6.0 \mathrm{~Hz}, 2 \mathrm{H}), 2.36(\mathrm{~s}, 3 \mathrm{H})$.

${ }^{13} \mathrm{C} \mathrm{NMR}\left(101 \mathrm{MHz}, \mathrm{CDCl}_{3}\right) \delta=196.3,161.6(\mathrm{~d}, J=124.23 \mathrm{~Hz}), 150.6,149.8,138.6,136.2(\mathrm{~d}, J=$ 8.08 Hz), 136.1, 134.6, 129.5, 129.1, 129.1(d, $J=4.5 \mathrm{~Hz}), 128.6(\mathrm{~d}, J=4.5 \mathrm{~Hz}), 126.2,125.2,124.6$, $122.7,116.6(\mathrm{~d}, J=1.01 \mathrm{~Hz}) 116.6,116.4,58.3,44.1,21.3$.

HRMS (ESI) calcd for [M+Na] ${ }^{+} \mathbf{C}_{\mathbf{2 3}} \mathbf{H}_{\mathbf{1 8}} \mathbf{F N O}_{\mathbf{3}} \mathbf{N a}^{+}, \mathrm{m} / \mathrm{z}$ : 398.1168, observed: 398.1166 .

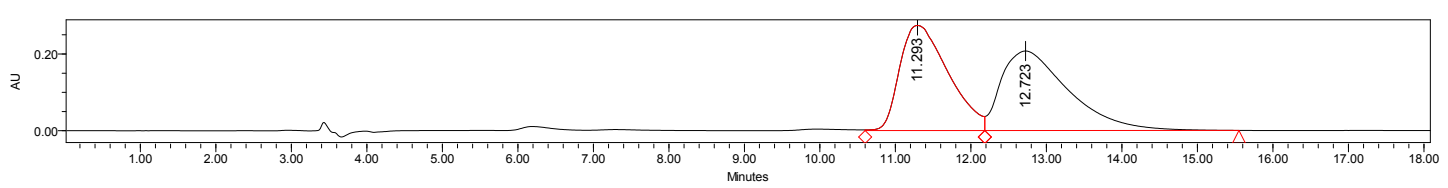

\begin{tabular}{|l|c|c|c|}
\hline & Retention Time & Area & $\%$ Area \\
\hline 1 & 11.293 & 12232712 & 48.86 \\
\hline 2 & 12.723 & 12803039 & 51.14 \\
\hline
\end{tabular}




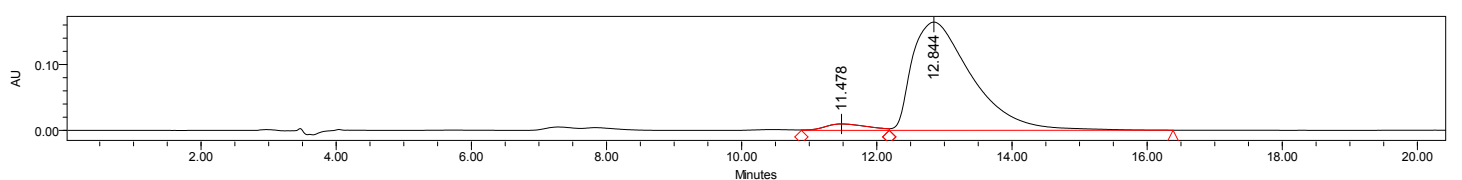

\begin{tabular}{|c|c|c|c|}
\hline & Retention Time & Area & $\%$ Area \\
\hline 1 & 11.478 & 423412 & 4.04 \\
\hline 2 & 12.844 & 10063872 & 95.96 \\
\hline
\end{tabular}

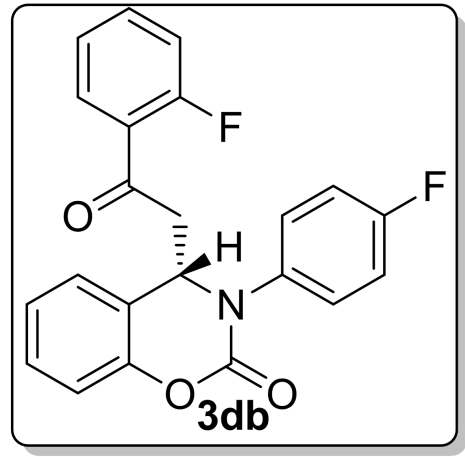

(S)-3-(4-fluorophenyl)-4-(2-(2-fluorophenyl)-2-oxoethyl)-4-methyl3,4-dihydro-2H-benzo[e][1,3] oxazin-2-one (3db)

White solid, $56 \mathrm{~h}, 15.0 \mathrm{mg}, 79 \%$ yield, $93 \%$ ee, $\mathrm{mp} 116-118{ }^{\circ} \mathrm{C}$; $[\alpha]_{\mathrm{D}}{ }^{18}=1.91\left(c: 0.2, \mathrm{CH}_{2} \mathrm{Cl}_{2}\right)$; Determined by HPLC analysis [Daicel chiralpak ODH, $n$-hexane $/ i$-PrOH $=70 / 30,1.0 \mathrm{~mL} / \mathrm{min}, \lambda=$ $254 \mathrm{~nm}, \mathrm{t}($ minor $)=10.62 \mathrm{~min}, \mathrm{t}($ major $)=12.46 \mathrm{~min}]$;

${ }^{1} \mathrm{H} \mathrm{NMR}\left(400 \mathrm{MHz} \mathrm{CDCl}_{3}\right) \delta=7.70(\mathrm{td}, J=7.6,1.6 \mathrm{~Hz}, 1 \mathrm{H})$, 7.50 (dddd, $J=8.4,7.2,5.2,1.8 \mathrm{~Hz}, 1 \mathrm{H}), 7.42-7.28$ (m, 4H), 7.22

$-7.02(\mathrm{~m}, 6 \mathrm{H}), 5.48(\mathrm{dd}, J=7.6,4.0 \mathrm{~Hz}, 1 \mathrm{H}), 3.63-3.43(\mathrm{~m}, 2 \mathrm{H})$.

${ }^{13} \mathrm{C}$ NMR $\left(101 \mathrm{MHz}, \mathrm{CDCl}_{3}\right) \delta=194.3(\mathrm{~d}, J=1.5 \mathrm{~Hz}), 163.0(\mathrm{~d}, J=13.6 \mathrm{~Hz}), 160.5(\mathrm{~d}, J=10.1$ $\mathrm{Hz}), 150.5,149.8,136.1(\mathrm{~d}, J=1.5 \mathrm{~Hz}), 135.4(\mathrm{~d}, J=4.5 \mathrm{~Hz}), 130.5$ (d, $J=1.0 \mathrm{~Hz}), 129.5,129.2(\mathrm{~d}, J$ $=4.5 \mathrm{~Hz}), 126.2,124.7(\mathrm{~d}, J=1.5 \mathrm{~Hz}), 124.6,122.4,116.9,116.7,116.6,116.7,116.6,116.4,58.1$, $49.0(\mathrm{~d}, J=4.0 \mathrm{~Hz})$.

HRMS (ESI) calcd for $[\mathbf{M}+\mathbf{N a}]^{+} \mathbf{C}_{\mathbf{2 2}} \mathbf{H}_{\mathbf{1 5}} \mathbf{F}_{\mathbf{2}} \mathbf{N O}_{3} \mathbf{N a}^{+}, \mathrm{m} / \mathrm{z}$ : 402.0918, observed: 402.0917 .

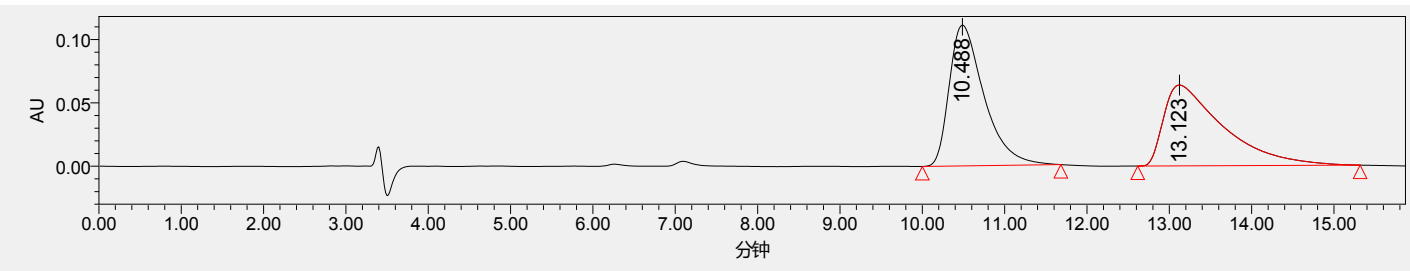

\begin{tabular}{|c|c|c|c|}
\hline & Retention Time & Area & $\%$ Area \\
\hline 1 & 10.488 & 3260710 & 50.50 \\
\hline 2 & 13.123 & 3195534 & 49.50 \\
\hline
\end{tabular}




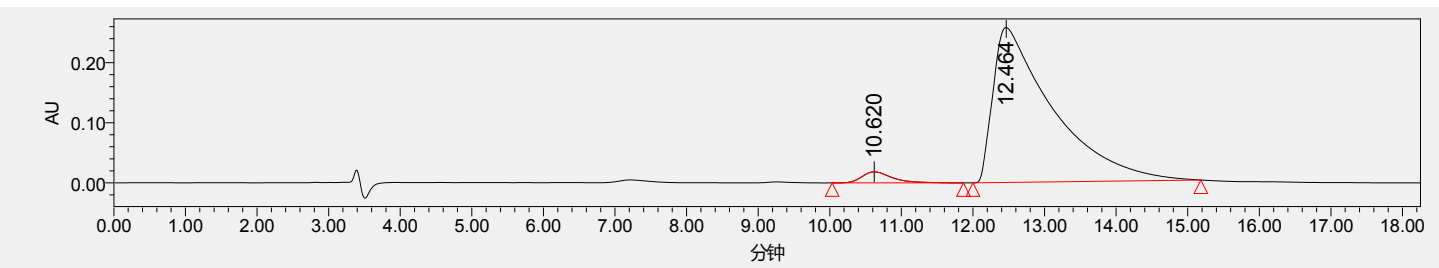

\begin{tabular}{|c|c|c|c|}
\hline & Retention Time & Area & $\%$ Area \\
\hline 1 & 10.620 & 541292 & 3.50 \\
\hline 2 & 12.464 & 14925822 & 96.50 \\
\hline
\end{tabular}

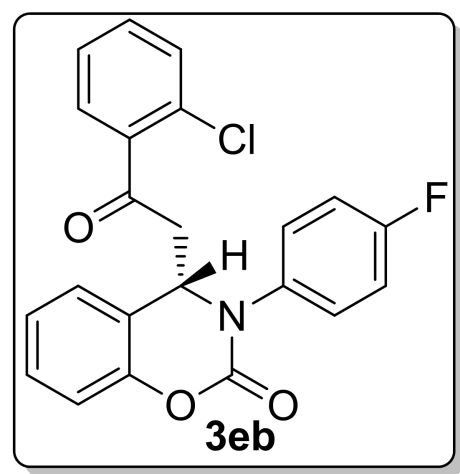

(S)-4-(2-(2-chlorophenyl)-2-oxoethyl)-3-(4-fluorophenyl)-3,4-dihydro2H-benzo[e][1,3]oxazin-2-one (3eb).

White solid, $98 \mathrm{~h}, 16.0 \mathrm{mg}, 81 \%$ yield, $89 \%$ ee, $\mathrm{mp} 80-82^{\circ} \mathrm{C} ;[\alpha]_{\mathrm{D}}{ }^{18}$ $=0.39\left(c: 0.7, \mathrm{CH}_{2} \mathrm{Cl}_{2}\right)$; Determined by HPLC analysis [Daicel chiralpak IA, $n$-hexane $/ i$-PrOH $=70 / 30,1.0 \mathrm{~mL} / \mathrm{min}, \lambda=254 \mathrm{~nm}, \mathrm{t}$ $($ minor $)=15.28 \mathrm{~min}, \mathrm{t}($ major $)=23.27 \mathrm{~min}$,$] ;$

${ }^{1} \mathrm{H}$ NMR $\left(400 \mathrm{MHz}, \mathrm{CDCl}_{3}\right) \delta=7.40(\mathrm{ddd}, J=10.4,5.2,2.8 \mathrm{~Hz}$, $2 \mathrm{H}), 7.38-7.28(\mathrm{~m}, 4 \mathrm{H}), 7.26-7.18(\mathrm{~m}, 2 \mathrm{H}), 7.18-7.09(\mathrm{~m}, 4 \mathrm{H})$, $5.46(\mathrm{t}, J=6.0 \mathrm{~Hz}, 1 \mathrm{H}), 3.60-3.46(\mathrm{~m}, 2 \mathrm{H})$.

${ }^{13} \mathrm{C}$ NMR $\left(101 \mathrm{MHz}, \mathrm{CDCl}_{3}\right) \delta=199.1,161.7(\mathrm{~d}, J=124.7 \mathrm{~Hz}), 150.4,149.8,138.1,136.0(\mathrm{~d}, J=$ $1.5 \mathrm{~Hz}), 132.6,131.2,130.7,129.6,129.4,129.2,129.2$ (d, $J=4.5 \mathrm{~Hz}), 127.1,126.1,124.6,122.1$, $116.7(\mathrm{~d}, J=3.0 \mathrm{~Hz}), 116.5,58.4,48.2$.

HRMS (ESI) calcd for $[\mathbf{M}+\mathbf{N a}]^{+} \mathbf{C}_{\mathbf{2 2}} \mathbf{H}_{15} \mathbf{C I F N O}_{3} \mathbf{N a}^{+}, \mathrm{m} / \mathrm{z}: 418.0622,420.0593$, observed: 418.0622, 420.0597.

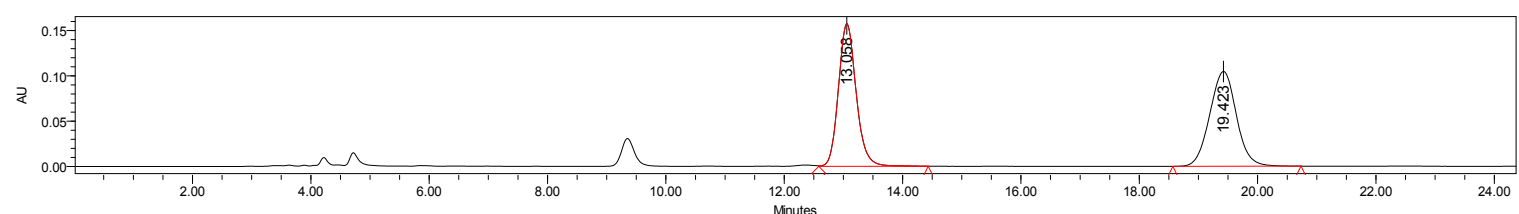

\begin{tabular}{|c|c|c|c|}
\hline & Retention Time & Area & $\%$ Area \\
\hline 1 & 15.277 & 585436 & 49.31 \\
\hline 2 & 23.266 & 601927 & 50.69 \\
\hline
\end{tabular}

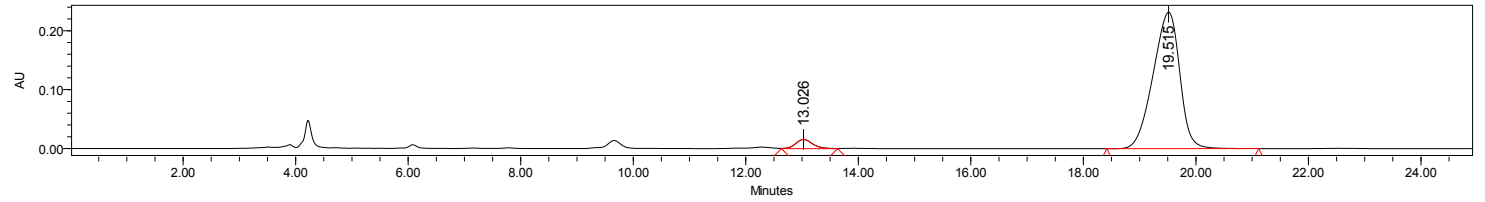

\begin{tabular}{|c|c|c|c|}
\hline & Retention Time & Area & $\%$ Area \\
\hline 1 & 15.078 & 3190678 & 5.52 \\
\hline
\end{tabular}




\begin{tabular}{|l|l|l|l|}
\hline 2 & 23.216 & 54570855 & 94.48 \\
\hline
\end{tabular}

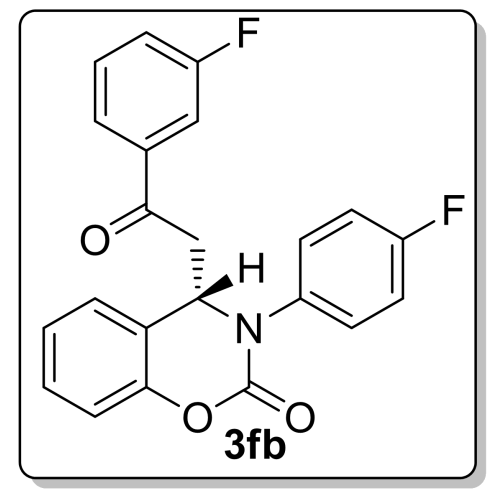

(S)-3-(4-fluorophenyl)-4-(2-(3-fluorophenyl)-2-oxoethyl)-3,4-dihydr o-2H-benzo[e][1,3]oxazin-2-one $(\mathbf{3 f b})$

White solid, $56 \mathrm{~h}, 15.2 \mathrm{mg}, 80 \%$ yield, $90 \%$ ee, $\mathrm{mp} 138-140{ }^{\circ} \mathrm{C}$; $[\alpha]_{\mathrm{D}}{ }^{18}=2.40\left(c: 0.1, \mathrm{CH}_{2} \mathrm{Cl}_{2}\right)$; Determined by HPLC analysis [Daicel chiralpak ODH $n$-hexane $/ i-\mathrm{PrOH}=80 / 20,1.0 \mathrm{~mL} / \mathrm{min}, \lambda=$ $254 \mathrm{~nm}, \mathrm{t}($ minor $)=13.93 \mathrm{~min}, \mathrm{t}($ major $)=15.63 \mathrm{~min}]$;

${ }^{1} \mathrm{H}$ NMR $\left(400 \mathrm{MHz}, \mathrm{CDCl}_{3}\right) \delta=7.59-7.52(\mathrm{~m}, 1 \mathrm{H}), 7.50-7.44$ (m, 1H), $7.43-7.35(\mathrm{~m}, 3 \mathrm{H}), 7.32(\mathrm{ddd}, J=7.2,4.0,1.6 \mathrm{~Hz}, 2 \mathrm{H})$, $7.28-7.21(\mathrm{~m}, 1 \mathrm{H}), 7.11(\mathrm{dq}, J=5.6,3.6 \mathrm{~Hz}, 4 \mathrm{H}), 5.49$ (dd, $J=7.6$, $4.0 \mathrm{~Hz}, 1 \mathrm{H}), 3.59-3.42(\mathrm{~m}, 2 \mathrm{H})$.

${ }^{13} \mathrm{C}$ NMR $\left(101 \mathrm{MHz}, \mathrm{CDCl}_{3}\right) \delta=195.0(\mathrm{~d}, J=1.0 \mathrm{~Hz}), 163.5(\mathrm{~d}, J=57.1 \mathrm{~Hz}), 161.0(\mathrm{~d}, J=57.1)$, 150.5, 149.8, 138.2 (d, $J=3.0 \mathrm{~Hz}), 136.0$ (d, $J=1.5 \mathrm{~Hz}), 130.5$ (d, $J=4.0 \mathrm{~Hz}), 129.6,129.1$ (d, $J=4.0$ Hz), 126.1, 124.7, 123.8 (d, $J=1.5 \mathrm{~Hz}), 122.3,121.0,120.7,116.7$ (d, $J=1.0 \mathrm{~Hz}) 116.5,114.9,114.6$, $58.2,44.1$.

HRMS (ESI) calcd for [M+Na] ${ }^{+} \mathbf{C}_{22} \mathbf{H}_{15} \mathbf{F}_{2} \mathbf{N O}_{3} \mathbf{N a}^{+}, \mathrm{m} / \mathrm{z}$ : 402.0918, observed: 402.0919 .

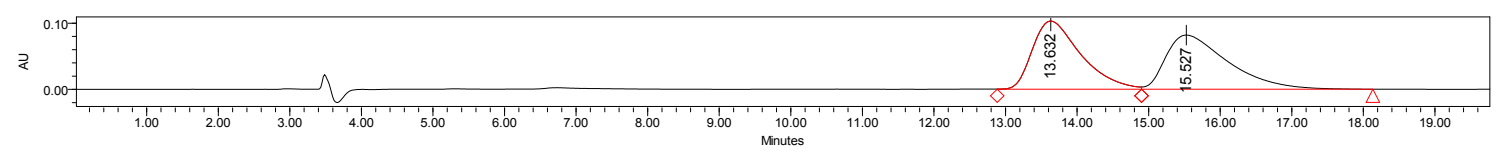

\begin{tabular}{|c|c|c|c|}
\hline & Retention Time & Area & $\%$ Area \\
\hline 1 & 13.632 & 4854004 & 49.61 \\
\hline 2 & 15.527 & 4930744 & 50.39 \\
\hline
\end{tabular}

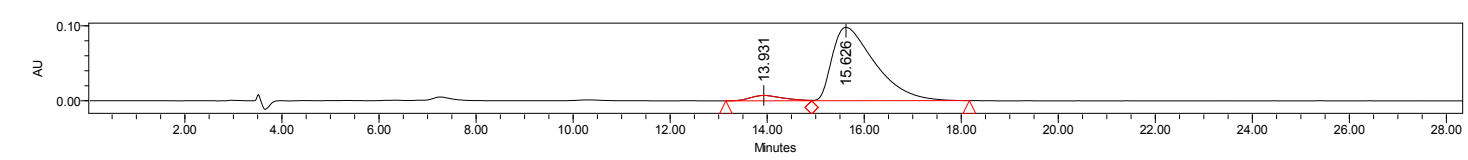

\begin{tabular}{|c|c|c|c|}
\hline & Retention Time & Area & $\%$ Area \\
\hline 1 & 13.931 & 327678 & 5.19 \\
\hline 2 & 15.626 & 5985360 & 94.81 \\
\hline
\end{tabular}

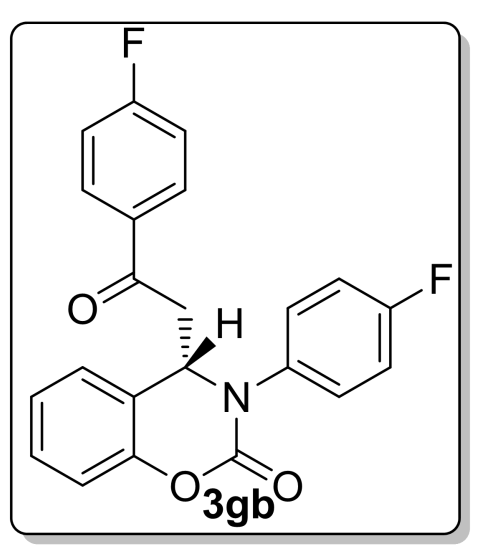

(S)-3-(4-fluorophenyl)-4-(2-(4-fluorophenyl)-2-oxoethyl)-3,4-dihydr o-2H-benzo[e][1,3] oxazin-2-one (3gb).

White solid, $89 \mathrm{~h}, 13.8 \mathrm{mg}, 73 \%$ yield, $94 \%$ ee, $\mathrm{mp} 152-154{ }^{\circ} \mathrm{C}$; $[\alpha]_{\mathrm{D}}{ }^{18}=2.06\left(c: 0.3, \mathrm{CH}_{2} \mathrm{Cl}_{2}\right)$; Determined by HPLC analysis [Daicel chiralpak ODH $n$-hexane $/ i$-PrOH $=80 / 20,1.0 \mathrm{~mL} / \mathrm{min}, \lambda=254 \mathrm{~nm}, \mathrm{t}$ $($ minor $)=14.30 \mathrm{~min}, \mathrm{t}($ major $)=15.66 \mathrm{~min}]$. 
${ }^{1} \mathrm{H}$ NMR $(400 \mathrm{MHz}, \mathrm{CDCl} 3) \delta=7.87-7.76(\mathrm{~m}, 2 \mathrm{H}), 7.44-7.35(\mathrm{~m}, 2 \mathrm{H}), 7.36-7.28(\mathrm{~m}, 2 \mathrm{H})$, $7.19-7.01(\mathrm{~m}, 6 \mathrm{H}), 5.49(\mathrm{dd}, \mathrm{J}=8.0,4.0 \mathrm{~Hz}, 1 \mathrm{H}), 3.49(\mathrm{qd}, J=16.8,6.0 \mathrm{~Hz}, 2 \mathrm{H})$.

${ }^{13} \mathrm{C}$ NMR $(101 \mathrm{MHz}, \mathrm{CDCl} 3) \delta=194.6,166.1(\mathrm{~d}, J=128.8 \mathrm{~Hz}),. 161.6(\mathrm{~d}, J=124.7 \mathrm{~Hz}), 150.5$, $149.8,136.0(\mathrm{~d}, J=1.5 \mathrm{~Hz}), 132.7(\mathrm{~d}, J=1.5 \mathrm{~Hz}), 130.7(\mathrm{~d}, J=5.0 \mathrm{~Hz}), 129.6,129.1(\mathrm{~d}, J=4.5 \mathrm{~Hz})$, 126.1, 124.6, 122.5, 116.7(d, $J=1.0 \mathrm{~Hz}), 116.5,116.0,115.8,58.3,43.8$.

HRMS (ESI) calcd for $[\mathbf{M}+\mathbf{N a}]^{+} \mathbf{C}_{22} \mathbf{H}_{15} \mathbf{F}_{2} \mathbf{N O}_{3} \mathbf{N a}^{+}, \mathrm{m} / \mathrm{z}$ : 402.0918, observed: 402.0918 .

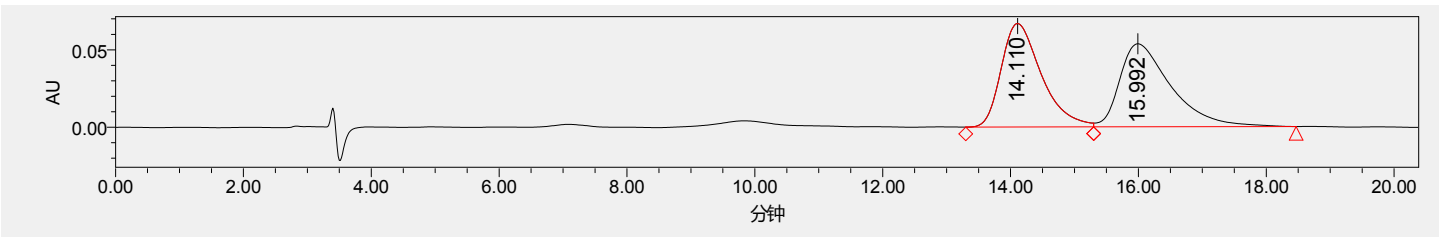

\begin{tabular}{|c|c|c|c|}
\hline & Retention Time & Area & $\%$ Area \\
\hline 1 & 14.110 & 2917919 & 49.44 \\
\hline 2 & 15.992 & 2983592 & 50.56 \\
\hline
\end{tabular}

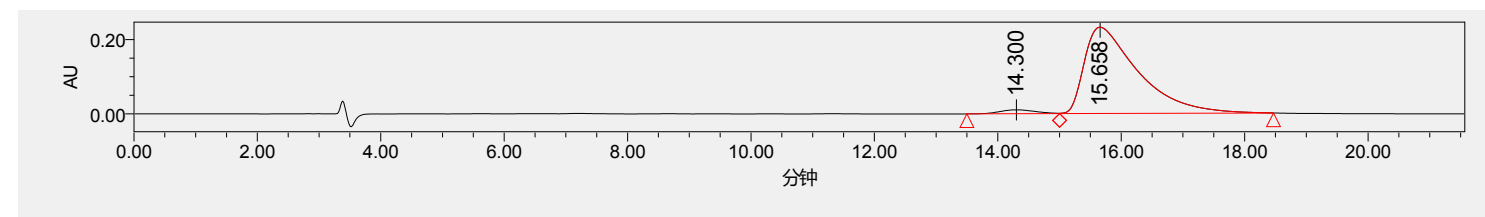

\begin{tabular}{|c|c|c|c|}
\hline & Retention Time & Area & $\%$ Area \\
\hline 1 & 14.300 & 424596 & 2.93 \\
\hline 2 & 15.658 & 14058469 & 97.07 \\
\hline
\end{tabular}

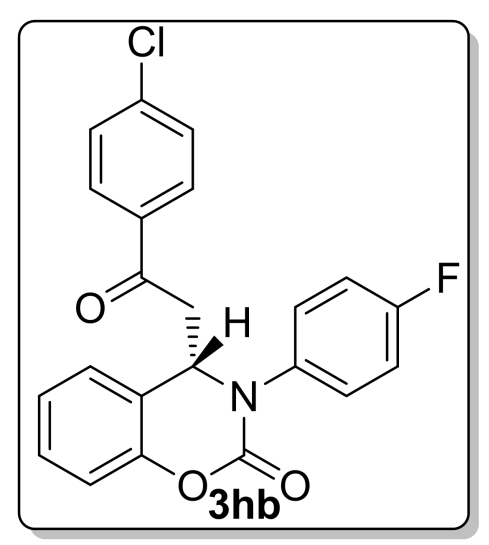

(S)-4-(2-(4-chlorophenyl)-2-oxoethyl)-3-(4-fluorophenyl)-3,4-dihydro2H-benzo[e][1,3] oxazin-2-one(3hb)

White solid, $96 \mathrm{~h}, 17.6 \mathrm{mg}, 89 \%$ yield, $89 \%$ ee, $\mathrm{mp} 160-162{ }^{\circ} \mathrm{C}$; $[\alpha]_{\mathrm{D}}{ }^{18}=1.68\left(c: 0.03, \mathrm{CH}_{2} \mathrm{Cl}_{2}\right)$; Determined by HPLC analysis [Daicel chiralpak IA, $n$-hexane $/ i$-PrOH $=80 / 20,1.0 \mathrm{~mL} / \mathrm{min}, \lambda=254$ $\mathrm{nm}, \mathrm{t}$ (minor) $=25.04 \mathrm{~min}, \mathrm{t}$ (major) $=43.34 \mathrm{~min}]$;

${ }^{1} \mathrm{H}$ NMR $\left(400 \mathrm{MHz}, \mathrm{CDCl}_{3}\right) \delta=7.77-7.67(\mathrm{~m}, 2 \mathrm{H}), 7.38$ (ddt, $J=5.6,4.4,2.4 \mathrm{~Hz}, 4 \mathrm{H}), 7.32(\mathrm{dd}, J=12.4,4.4 \mathrm{~Hz}, 2 \mathrm{H}), 7.18-$ 7.04 (m, 4H), 5.48 (dd, $J=7.6,4.0 \mathrm{~Hz}, 1 \mathrm{H}), 3.49$ (qd, $J=16.8,6.0$

$\mathrm{Hz}, 2 \mathrm{H})$.

${ }^{13} \mathrm{C}$ NMR $\left(101 \mathrm{MHz}, \mathrm{CDCl}_{3}\right) \delta=195.0,161.6(\mathrm{~d}, J=124.2 \mathrm{~Hz}) 150.5,149.8,140.3,136.0(\mathrm{~d}, J=$ $1.5 \mathrm{~Hz}), 136.0,134.5,129.6,129.4,129.1,129.1,129.0,126.1,124.7,122.4,116.7$ (d, $J=1.0 \mathrm{~Hz})$, $116.5,58.3,43.9$.

HRMS (ESI) calcd for $[\mathbf{M}+\mathbf{N a}]^{+} \mathbf{C}_{22} \mathbf{H}_{15} \mathbf{C I F N O}_{3} \mathbf{N a}^{+}, \mathrm{m} / \mathrm{z}$ : 418.0622, 420.0593; observed: 
418.0696, 464.0593.

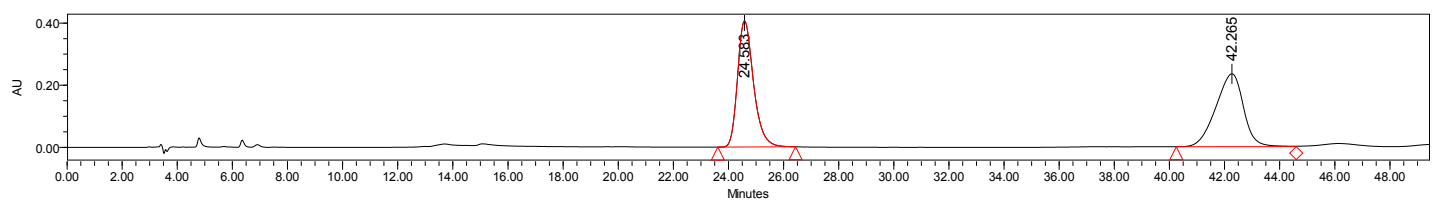

\begin{tabular}{|l|c|c|c|}
\hline & Retention Time & Area & $\%$ Area \\
\hline 1 & 24.583 & 16327955 & 49.97 \\
\hline 2 & 42.265 & 16345504 & 50.03 \\
\hline
\end{tabular}

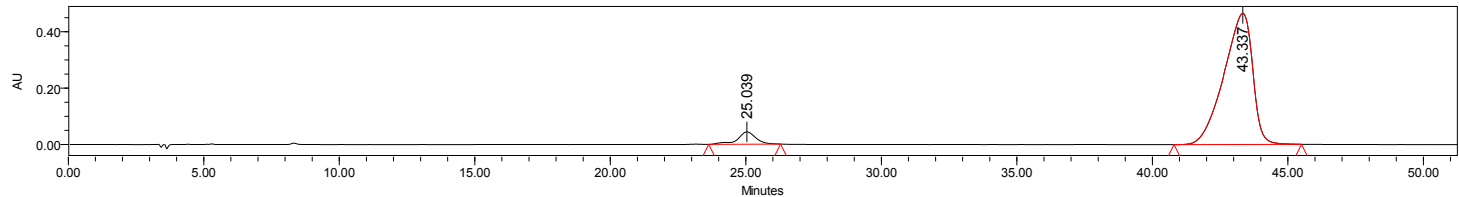

\begin{tabular}{|l|c|c|c|}
\hline & Retention Time & Area & $\%$ Area \\
\hline 1 & 25.039 & 2058836 & 5.67 \\
\hline 2 & 43.337 & 34268740 & 94.33 \\
\hline
\end{tabular}

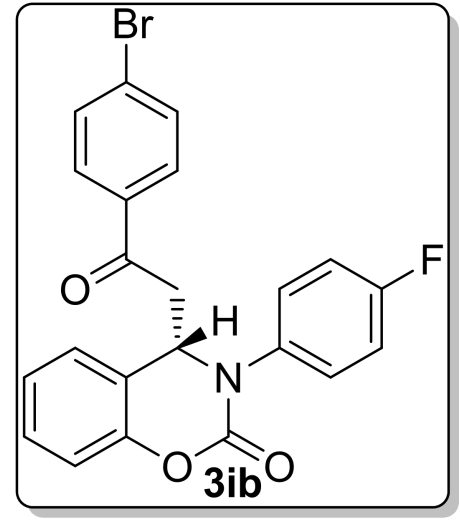

(S)-4-(2-(4-bromophenyl)-2-oxoethyl)-3-(4-fluorophenyl)-3,4-dihydro2H-benzo[e][1,3]oxazin-2-one(3ib).

White solid, $88 \mathrm{~h}, 17.7 \mathrm{mg}, 80 \%$ yield, 91\% ee, $\mathrm{mp} 174-176{ }^{\circ} \mathrm{C}$; $[\alpha]_{\mathrm{D}}{ }^{18}=2.19\left(c: 0.05, \mathrm{CH}_{2} \mathrm{Cl}_{2}\right)$; Determined by HPLC analysis [Daicel chiralpak IA, $n$-hexane $/ i$-PrOH $=70 / 30,1.0 \mathrm{~mL} / \mathrm{min}, \lambda=254 \mathrm{~nm}, \mathrm{t}$ $($ minor $)=17.67 \mathrm{~min}, \mathrm{t}($ major $)=25.79 \mathrm{~min}]$;

${ }^{1} \mathrm{H}$ NMR $\left(400 \mathrm{MHz}, \mathrm{CDCl}_{3}\right) \delta 7.67-7.60(\mathrm{~m}, 2 \mathrm{H}), 7.58-7.51(\mathrm{~m}$, 2H), $7.42-7.35(\mathrm{~m}, 2 \mathrm{H}), 7.34-7.27(\mathrm{~m}, 2 \mathrm{H}), 7.18-7.07(\mathrm{~m}, 4 \mathrm{H})$, $5.48(\mathrm{dd}, J=7.6,4.0 \mathrm{~Hz}, 1 \mathrm{H}), 3.48(\mathrm{qd}, J=17.2,6.0 \mathrm{~Hz}, 2 \mathrm{H})$.

${ }^{13} \mathrm{C} \mathrm{NMR}\left(101 \mathrm{MHz}, \mathrm{CDCl}_{3}\right) \delta=195.2,161.7,(\mathrm{~d}, J=124.7 \mathrm{~Hz}), 150.5,149.8,136.0(\mathrm{~d}, J=1.5 \mathrm{~Hz})$, 134.9, 132.1, 129.6, 129.5, 129.1, 129.0, 126.1, 124.7, 122.4, 116.7 (d, $J=1.0 \mathrm{~Hz}), 116.5,58.3,43.9$.

HRMS (ESI) calcd for $[\mathbf{M}+\mathbf{N a}]^{+} \mathbf{C}_{\mathbf{2 2}} \mathbf{H}_{15} \mathbf{B r F N O}_{3} \mathbf{N a}^{+}, \mathrm{m} / \mathrm{z}:$ 462.0117, 464.0097; observed: 462.0119, 464.0098.

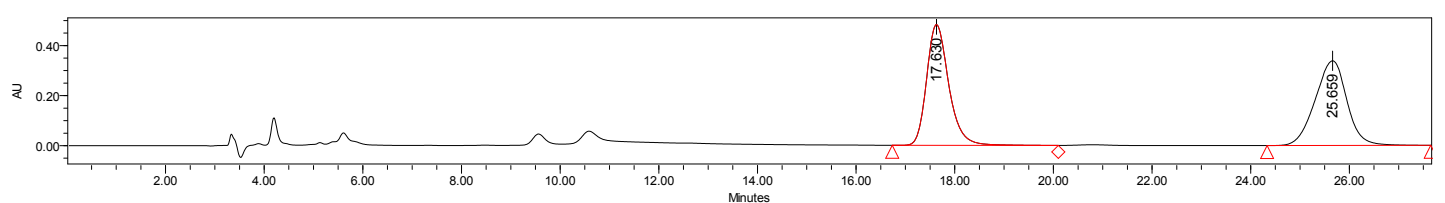

\begin{tabular}{|c|c|c|c|}
\hline & Retention Time & Area & $\%$ Area \\
\hline 1 & 17.630 & 15059243 & 49.92 \\
\hline 2 & 25.659 & 15105369 & 50.08 \\
\hline
\end{tabular}




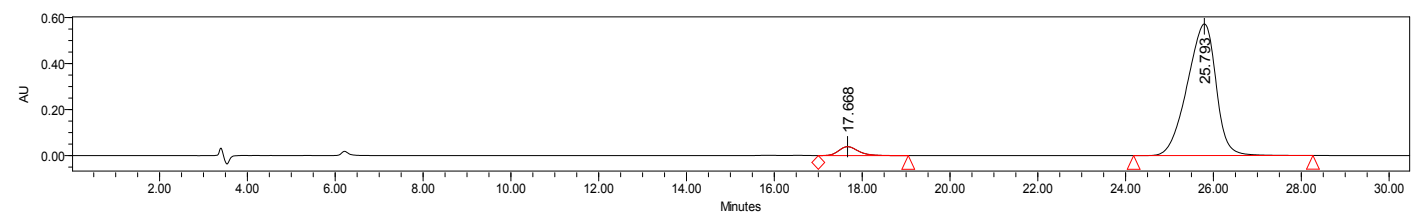

\begin{tabular}{|c|c|c|c|}
\hline & Retention Time & Area & $\%$ Area \\
\hline 1 & 17.668 & 1198514 & 4.41 \\
\hline 2 & 25.793 & 25979468 & 95.59 \\
\hline
\end{tabular}

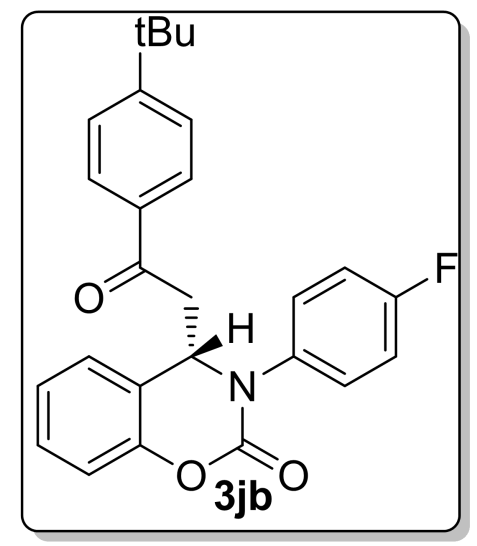

9H).

${ }^{13} \mathrm{C}$ NMR $\left(101 \mathrm{MHz}, \mathrm{CDCl}_{3}\right) \delta=195.7,161.6(\mathrm{~d}, J=124.2 \mathrm{~Hz}), 157.7,150.6,149.8,136.1(\mathrm{~d}, J=$ $2.0 \mathrm{~Hz}$ ), 133.7, 129.4, 129.1, 129.0, 128.0, 126.2, 125.7, 124.6, 122.7, 116.6 (d, $J=1.5 \mathrm{~Hz}$ ), 116.4, 58.2, 43.9, 35.2, 31.0.

HRMS (ESI) calcd for $[\mathbf{M}+\mathbf{N a}]^{+} \mathbf{C}_{26} \mathbf{H}_{24} \mathbf{F N O}_{3} \mathbf{N a}^{+}, \mathrm{m} / \mathrm{z}$ : 440.1638, observed: 440.1638.

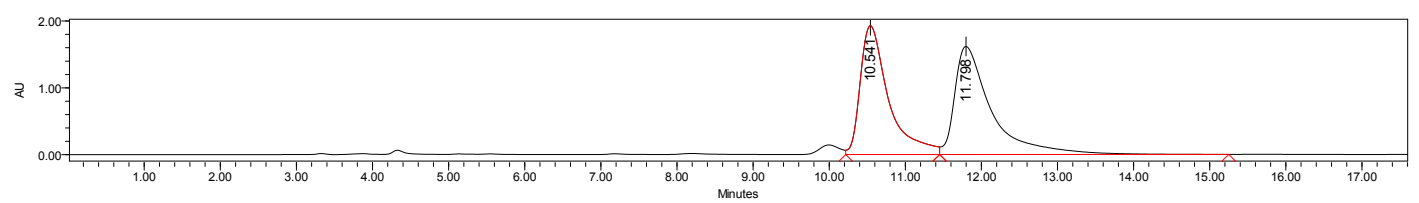

\begin{tabular}{|l|c|c|c|}
\hline & Retention Time & Area & $\%$ Area \\
\hline 1 & 10.541 & 49793087 & 47.84 \\
\hline 2 & 11.798 & 54291942 & 52.16 \\
\hline
\end{tabular}

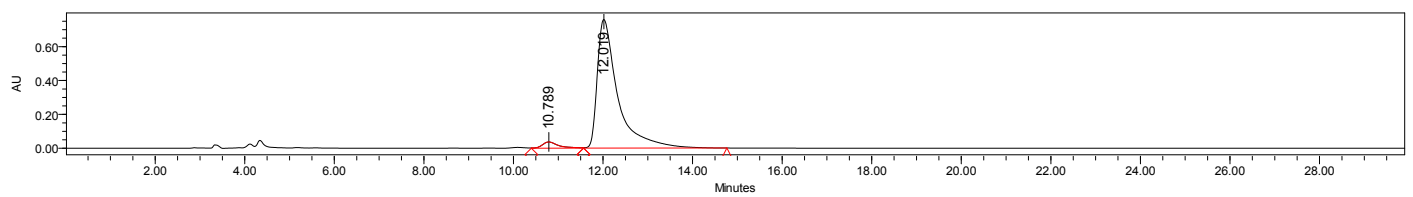

\begin{tabular}{|c|c|c|c|}
\hline & Retention Time & Area & $\%$ Area \\
\hline 1 & 10.789 & 914196 & 3.65 \\
\hline 2 & 12.019 & 24101075 & 96.35 \\
\hline
\end{tabular}




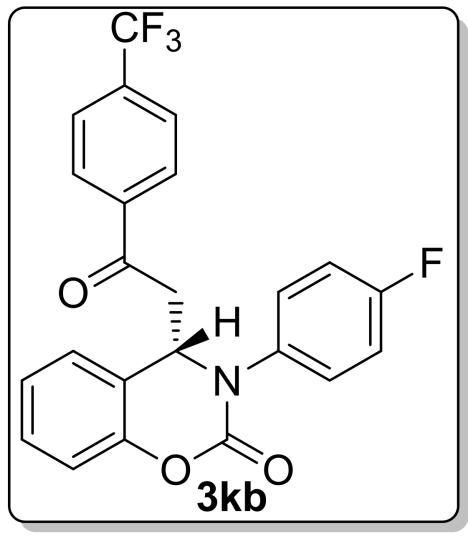

(S)-3-(4-fluorophenyl)-4-(2-oxo-2-(4-(trifluoromethyl)phenyl)ethyl)3,4-dihydro-2H-benzo[e][1,3] oxazin-2-one (3kb).

White solid, $40 \mathrm{~h}, 21.2 \mathrm{mg}$, 99\% yield, $91 \%$ ee, mp 158-160 ${ }^{\circ} \mathrm{C}$; $[\alpha]_{\mathrm{D}}^{18}=1.91\left(c: 0.07, \mathrm{CH}_{2} \mathrm{Cl}_{2}\right)$; Determined by HPLC analysis [Daicel chiralpak IA, $n$-hexane $/ i-\mathrm{PrOH}=70 / 30,1.0 \mathrm{~mL} / \mathrm{min}, \lambda=254$ $\mathrm{nm}, \mathrm{t}($ minor $)=13.34 \mathrm{~min}, \mathrm{t}($ major $)=14.87 \mathrm{~min}]$;

${ }^{1} \mathrm{H}$ NMR $\left(400 \mathrm{MHz}, \mathrm{CDCl}_{3}\right) \delta=7.88(\mathrm{~d}, J=8.4 \mathrm{~Hz}, 2 \mathrm{H}), 7.67(\mathrm{~d}, J$ $=8.4 \mathrm{~Hz}, 2 \mathrm{H}), 7.45-7.36(\mathrm{~m}, 2 \mathrm{H}), 7.32(\mathrm{dd}, J=12.0,4.4 \mathrm{~Hz}, 2 \mathrm{H})$, $7.20-7.06(\mathrm{~m}, 4 \mathrm{H}), 5.50(\mathrm{dd}, J=7.6,4.0 \mathrm{~Hz}, 1 \mathrm{H}), 3.63-3.45(\mathrm{~m}$,

2H).

${ }^{13} \mathrm{C}$ NMR $\left(101 \mathrm{MHz}, \mathrm{CDCl}_{3}\right) \delta=195.5,161.7(\mathrm{~d}, J=124.7 \mathrm{~Hz}), 150.5,149.7,138.8,135.9(\mathrm{~d}, J=$ $2.0 \mathrm{~Hz}) 135.2,134.9,129.7,129.1$ (d, $J=4.5 \mathrm{~Hz}), 128.4,126.0,125.8$ (d, $J=2.0 \mathrm{~Hz}), 124.8,122.0$, $116.8(\mathrm{~d}, J=4.0 \mathrm{~Hz}) 116.6,58.2,44.1$.

HRMS (ESI) calcd for $[\mathbf{M}+\mathbf{N a}]^{+} \mathbf{C}_{23} \mathbf{H}_{15} \mathbf{F}_{4} \mathbf{N O}_{3} \mathbf{N a}^{+}, \mathrm{m} / \mathrm{z}$ : 452.0886 , observed: 452.0883 .

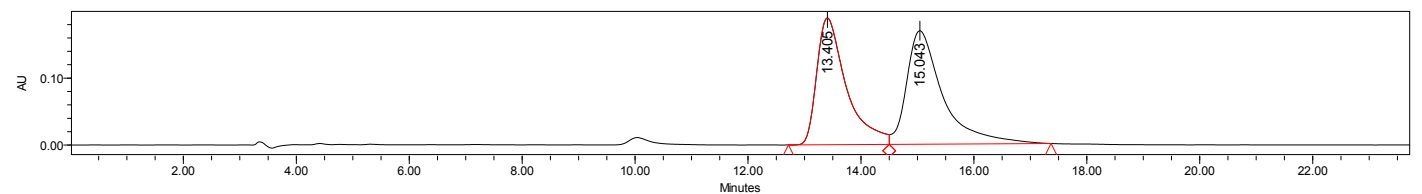

\begin{tabular}{|l|c|c|c|}
\hline & Retention Time & Area & $\%$ Area \\
\hline 1 & 13.405 & 6902421 & 47.77 \\
\hline 2 & 15.043 & 7547839 & 52.23 \\
\hline
\end{tabular}

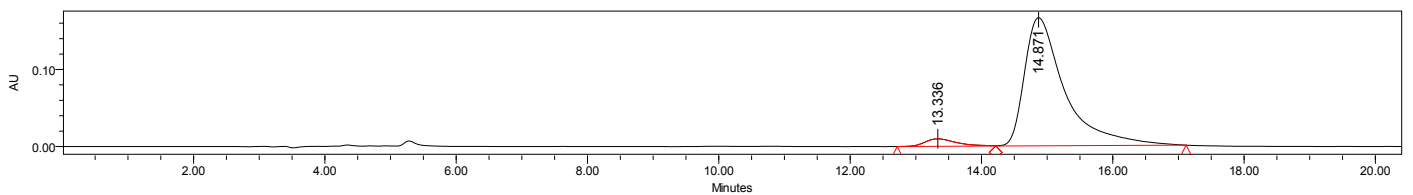

\begin{tabular}{|c|c|c|c|}
\hline & Retention Time & Area & $\%$ Area \\
\hline 1 & 13.336 & 326542 & 4.41 \\
\hline 2 & 14.871 & 7084554 & 95.59 \\
\hline
\end{tabular}

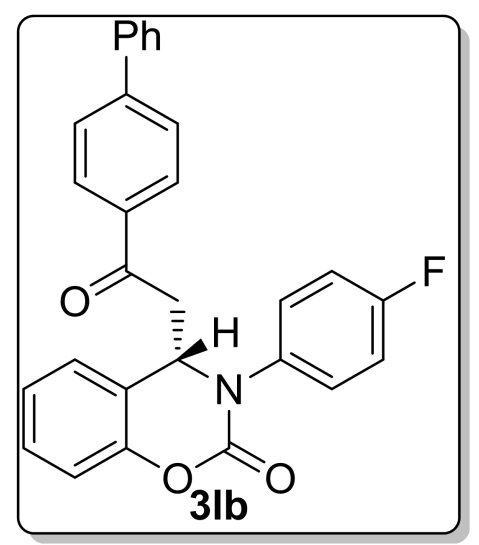

(S)-4-(2-([1,1'-biphenyl]-4-yl)-2-oxoethyl)-3-(4-fluorophenyl)-3,4-dih ydro-2H-benzo[e][1,3]oxazin-2-one (3lb).

White solid, 88 h, $20.3 \mathrm{mg}, 99 \%$ yield, 95\% ee; mp $216-218{ }^{\circ} \mathrm{C}$; $[\alpha]_{\mathrm{D}}{ }^{18}=2.62\left(c: 0.3, \mathrm{CH}_{2} \mathrm{Cl}_{2}\right)$; Determined by HPLC analysis [Daicel chiralpak IA, $n$-hexane $/ i$-PrOH $=70 / 30,1.0 \mathrm{~mL} / \mathrm{min}, \lambda=254 \mathrm{~nm}, \mathrm{t}$ $($ minor $)=24.17 \mathrm{~min}, \mathrm{t}($ major $)=33.97 \mathrm{~min}$,$] ;$ 
${ }^{1} \mathrm{H}$ NMR $\left(400 \mathrm{MHz}, \mathrm{CDCl}_{3}\right) \delta=7.86(\mathrm{~d}, J=8.4 \mathrm{~Hz}, 2 \mathrm{H}), 7.69-7.54(\mathrm{~m}, 4 \mathrm{H}), 7.51-7.37(\mathrm{~m}, 5 \mathrm{H})$, $7.37-7.28(\mathrm{~m}, 2 \mathrm{H}), 7.19-7.04(\mathrm{~m}, 4 \mathrm{H}), 5.53(\mathrm{dd}, J=8.0,4.0 \mathrm{~Hz}, 1 \mathrm{H}), 3.56(\mathrm{qd}, J=16.8,6.0 \mathrm{~Hz}$, $2 \mathrm{H})$.

${ }^{13} \mathrm{C}$ NMR $\left(101 \mathrm{MHz}, \mathrm{CDCl}_{3}\right) \delta=195.7,161.6(\mathrm{~d}, J=124.7 \mathrm{~Hz}), 150.6,149.8,146.5,139.5,136.1$, $134.9,129.5$, 129.1, 129.0, 128.6, 128.5, 127.3, 127.3, 126.2, 124.6, 122.6, 116.7 (d, J=1.5 Hz), 116.5, $58.3,44.0$.

HRMS (ESI) calcd for $[\mathbf{M}+\mathbf{N a}]^{+} \mathbf{C}_{28} \mathbf{H}_{20} \mathbf{F N O}_{3} \mathbf{N a}^{+}, \mathrm{m} / \mathrm{z}: 460.1325$, observed: 460.1325 .

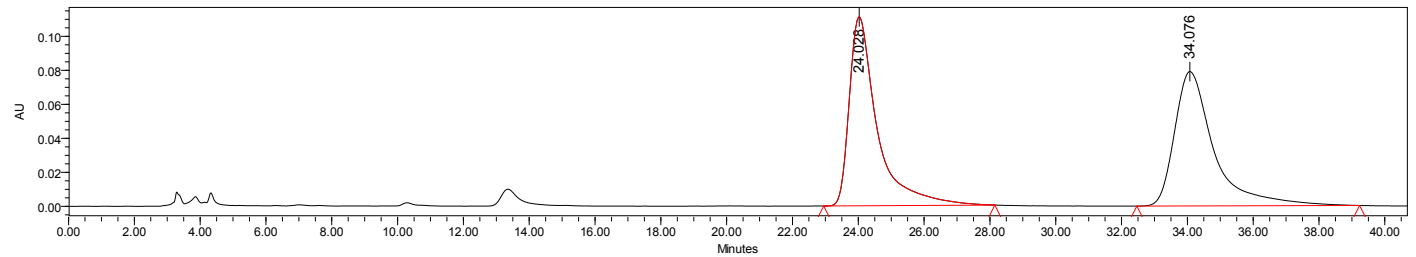

\begin{tabular}{|c|c|c|c|}
\hline & Retention Time & Area & $\%$ Area \\
\hline 1 & 24.028 & 6554201 & 50.05 \\
\hline 2 & 34.076 & 6541419 & 49.95 \\
\hline
\end{tabular}

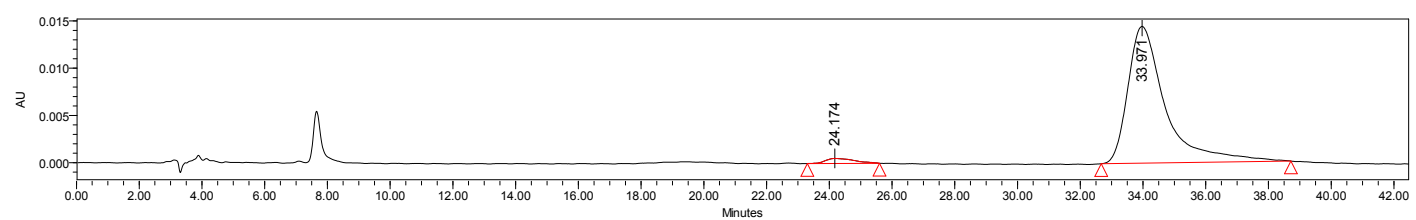

\begin{tabular}{|c|c|c|c|}
\hline & Retention Time & Area & $\%$ Area \\
\hline 1 & 24.174 & 33773 & 2.74 \\
\hline 2 & 33.971 & 1198470 & 97.26 \\
\hline
\end{tabular}

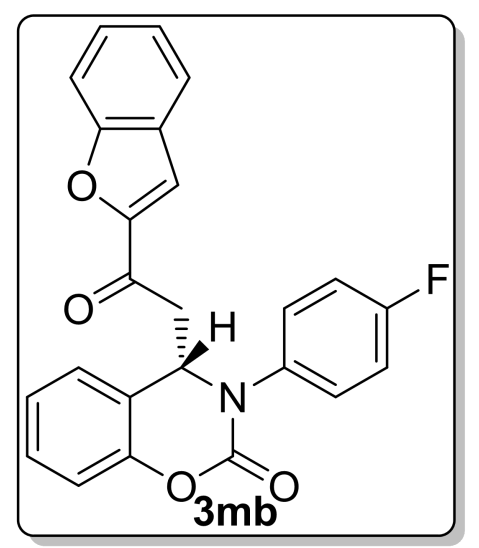

$160.5,155.7,151.9,150.4,149.8,136.0,135.9,129.7,129.2$ (d, $J=4.0 \mathrm{~Hz}), 128.9,126.7,126.1,124.7$ $124.2,123.5,122.1,116.7,116.5,113.7,112.5,58.2,44.4$.

HRMS (ESI) calcd for $[\mathbf{M}+\mathbf{N a}]^{+} \mathbf{C}_{\mathbf{2 5}} \mathbf{H}_{\mathbf{1 7}} \mathbf{F} \mathbf{N O}_{4} \mathbf{N a}^{+}, \mathrm{m} / \mathrm{z}: 424.0961$, observed:424.0957.
(S)-4-(2-(benzofuran-2-yl)-2-oxoethyl)-3-(4-fluorophenyl)-3,4-dihydro -2H-benzo[e][1,3]oxazin-2-one (3mb).

White solid, $56 \mathrm{~h}, 19.9 \mathrm{mg}, 99 \%$ yield, 89\% ee; mp $118-120{ }^{\circ} \mathrm{C}$; $[\alpha]_{\mathrm{D}}^{18}=2.62\left(c: 0.3, \mathrm{CH}_{2} \mathrm{Cl}_{2}\right)$; Determined by HPLC analysis [Daicel chiralpak IA, $n$-hexane $/ i$-PrOH $=70 / 30,1.0 \mathrm{~mL} / \mathrm{min}, \lambda=254 \mathrm{~nm}, \mathrm{t}$ $($ minor $)=15.93 \mathrm{~min}, \mathrm{t}($ major $)=21.06 \mathrm{~min}$,$] ;$

${ }^{1} \mathrm{H}$ NMR $\left(400 \mathrm{MHz}, \mathrm{CDCl}_{3}\right) \delta=7.59(\mathrm{~d}, J=8.0 \mathrm{~Hz}, 1 \mathrm{H}), 7.47-$ $7.37(\mathrm{~m}, 2 \mathrm{H}), 7.37-7.29(\mathrm{~m}, 3 \mathrm{H}), 7.28-7.20(\mathrm{~m}, 3 \mathrm{H}), 7.11-6.97$ (m, 4H), 5.42 (t, $J=6.0 \mathrm{~Hz}, 1 \mathrm{H}), 3.45$ (d, $J=6.0 \mathrm{~Hz}, 2 \mathrm{H})$.

${ }^{13} \mathrm{C} \mathrm{NMR}\left(101 \mathrm{MHz}, \mathrm{CDCl}_{3}\right) \delta=187.0,161.7(\mathrm{~d}, J=124.7 \mathrm{~Hz})$, 


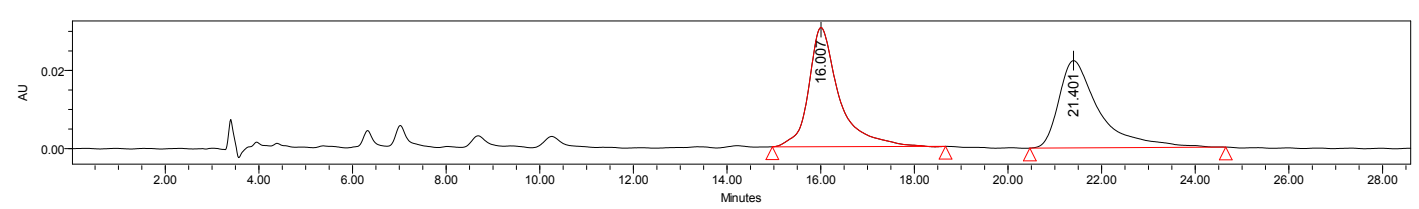

\begin{tabular}{|l|c|c|c|}
\hline & Retention Time & Area & $\%$ Area \\
\hline 1 & 16.007 & 1380476 & 50.86 \\
\hline 2 & 21.401 & 1333878 & 49.14 \\
\hline
\end{tabular}

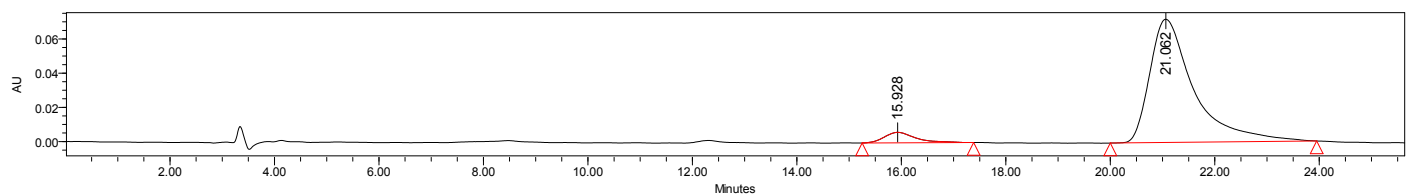

\begin{tabular}{|c|c|c|c|}
\hline & Retention Time & Area & $\%$ Area \\
\hline 1 & 15.928 & 251607 & 5.64 \\
\hline 2 & 21.062 & 4212403 & 94.36 \\
\hline
\end{tabular}

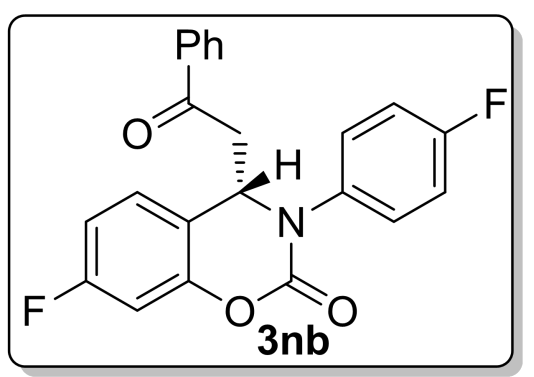

7.52 (m, 1H), $7.49-7.35$ (m, 4H), 7.32 (dd, $J=8.4,6.0 \mathrm{~Hz}, 1 \mathrm{H}), 7.18-7.07$ (m, 2H), $6.92-6.78$ (m, 2H), 5.47 (dd, $J=8.4,3.6 \mathrm{~Hz}, 1 \mathrm{H}), 3.51$ (qd, $J=17.2,6.0 \mathrm{~Hz}, 2 \mathrm{H})$.

${ }^{13} \mathrm{C}$ NMR $\left(101 \mathrm{MHz}, \mathrm{CDCl}_{3}\right) \delta=196.1,163.5(\mathrm{~d}, J=55.0 \mathrm{~Hz}), 161.1(\mathrm{~d}, J=55.0 \mathrm{~Hz}), 150.6(\mathrm{~d}, J$ $=6.1 \mathrm{~Hz}), 150.6,150.0,136.1,135.8(\mathrm{~d}, J=1.5 \mathrm{~Hz}), 133.9,129.0(\mathrm{~d}, J=4.5 \mathrm{~Hz}), 128.8,128.0,127.5(\mathrm{~d}$, $J=5.1 \mathrm{~Hz}), 118.5(\mathrm{~d}, J=1.5 \mathrm{~Hz}), 116.7(\mathrm{~d}, J=11.1 \mathrm{~Hz}), 111.8(\mathrm{~d}, J=11.1 \mathrm{~Hz}), 104.5(\mathrm{~d}, J=13.1 \mathrm{~Hz})$, $57.9,43.8$.

HRMS (ESI) calcd for $[\mathbf{M}+\mathbf{N a}]^{+} \mathbf{C}_{22} \mathbf{H}_{15} \mathbf{F}_{2} \mathbf{N O}_{3} \mathbf{N a}^{+}$, m/z: 402.0918, observed: 402.0915;

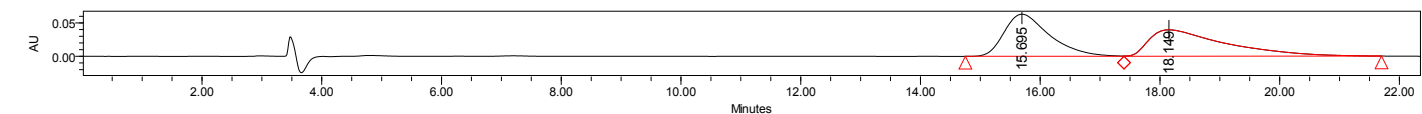

\begin{tabular}{|l|c|c|c|}
\hline & Retention Time & Area & $\%$ Area \\
\hline 1 & 15.695 & 3322752 & 48.78 \\
\hline 2 & 18.149 & 3489099 & 51.22 \\
\hline
\end{tabular}

(S)-7-fluoro-3-(4-fluorophenyl)-4-(2-oxo-2-phenylethyl)-3,4-dih ydro-2H-benzo[e][1,3]oxazin-2-one (3nb).

White solid, $78 \mathrm{~h}, 18.7 \mathrm{mg}$, 99\% yield, $85 \%$ ee, mp 178-180 ${ }^{\circ} \mathrm{C} ;[\alpha]_{\mathrm{D}}{ }^{18}=1.63\left(c: 0.1, \mathrm{CH}_{2} \mathrm{Cl}_{2}\right)$; Determined by HPLC analysis [Daicel chiralpak ODH, $n$-hexane $/ i-\mathrm{PrOH}=80 / 20,1.0 \mathrm{~mL} / \mathrm{min}$, $\lambda=254 \mathrm{~nm}, \mathrm{t}$ (minor) $=15.81 \mathrm{~min}, \mathrm{t}$ (major) $=18.18 \mathrm{~min}$,$] ;$

${ }^{1} \mathrm{H}$ NMR $\left(400 \mathrm{MHz}, \mathrm{CDCl}_{3}\right) \delta=7.87-7.74(\mathrm{~m}, 2 \mathrm{H}), 7.61-$ 


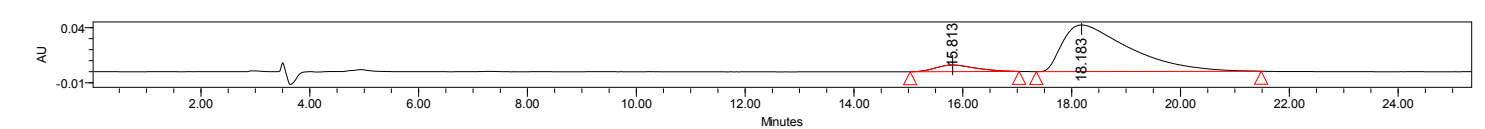

\begin{tabular}{|c|c|c|c|}
\hline & Retention Time & Area & $\%$ Area \\
\hline 1 & 15.813 & 304194 & 7.78 \\
\hline 2 & 18.183 & 3608244 & 92.22 \\
\hline
\end{tabular}

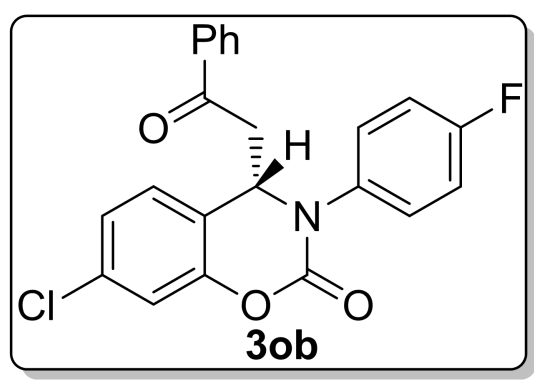

(S)-7-chloro-3-(4-fluorophenyl)-4-(2-oxo-2-phenylethyl)-3,4-dih ydro-2H-benzo[e][1,3]oxazin-2-one (3ob).

White solid, 78 h, $19.6 \mathrm{mg}$, 99\% yield, 86\% ee, mp 156-158 ${ }^{\circ} \mathrm{C} ;[\alpha]_{\mathrm{D}}{ }^{18}=2.40\left(c: 0.1, \mathrm{CH}_{2} \mathrm{Cl}_{2}\right)$; Determined by HPLC analysis [Daicel chiralpak ODH, $n$-hexane $/ i$-PrOH $=80 / 20,1.0 \mathrm{~mL} / \mathrm{min}, \lambda$ $=254 \mathrm{~nm}, \mathrm{t}($ minor $)=18.77 \mathrm{~min}, \mathrm{t}$ (major) $=25.11 \mathrm{~min}$, ]

${ }^{1} \mathrm{H}$ NMR $\left(400 \mathrm{MHz}, \mathrm{CDCl}_{3}\right) \delta=7.78-7.66(\mathrm{~m}, 2 \mathrm{H}), 7.54-$ $7.44(\mathrm{~m}, 1 \mathrm{H}), 7.38-7.27$ (m, 4H), $7.25-7.17(\mathrm{~m}, 2 \mathrm{H}), 7.11-6.97$ (m, 4H), 5.40 (dd, $J=8.4,3.6 \mathrm{~Hz}$, $1 \mathrm{H}), 3.44$ (qd, $J=17.2,6.0 \mathrm{~Hz}, 2 \mathrm{H})$.

${ }^{13} \mathrm{C}$ NMR $\left(101 \mathrm{MHz}, \mathrm{CDCl}_{3}\right) \delta=196.0,161.7(\mathrm{~d}, J=124.7 \mathrm{~Hz}), 150.3,149.9,136.0,135.8(\mathrm{~d}, J=$ $1.5 \mathrm{~Hz}$ ), 134.9, 134.0, 129.0 (d, $J=4.0 \mathrm{~Hz}), 128.8,128.0,127.3,124.9,121.1,117.0,116.8,116.6$, $57.9,43.7$.

HRMS (ESI) calcd for [M+Na] ${ }^{+} \mathbf{C}_{22} \mathbf{H}_{15} \mathbf{C l F N O}_{3} \mathbf{N a}^{+}, \mathrm{m} / \mathrm{z}$ : 418.0622,420.0593, observed: 418.0607, 420.0598

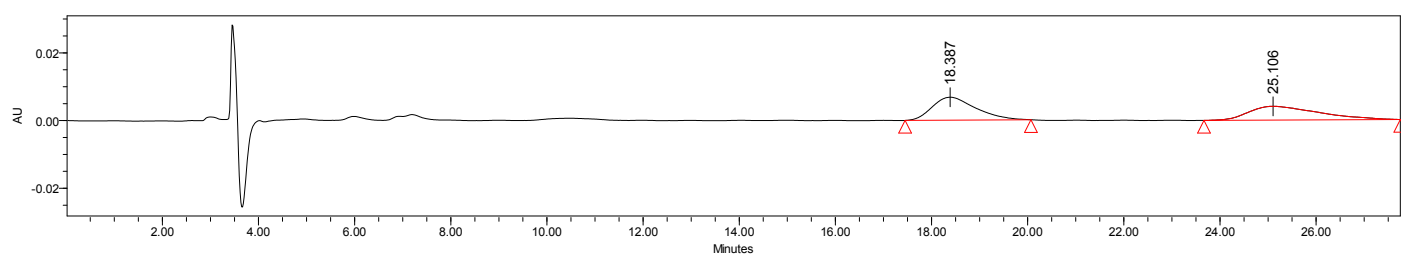

\begin{tabular}{|c|c|c|c|}
\hline & Retention Time & Area & $\%$ Area \\
\hline 1 & 18.387 & 425121 & 50.79 \\
\hline 2 & 25.106 & 411870 & 49.21 \\
\hline
\end{tabular}

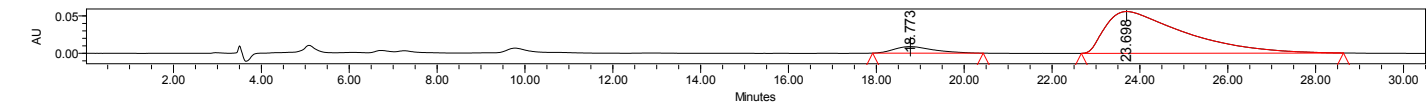

\begin{tabular}{|c|c|c|c|}
\hline & Retention Time & Area & $\%$ Area \\
\hline 1 & 18.773 & 540917 & 7.17 \\
\hline 2 & 23.698 & 6999186 & 92.83 \\
\hline
\end{tabular}


<smiles>O=C(C[C@H]1c2ccc(Br)cc2OC(=O)N1c1ccc(F)cc1)c1ccccc1</smiles>

(S)-7-bromo-3-(4-fluorophenyl)-4-(2-oxo-2-phenylethyl)-3,4dihydro-2H-benzo[e][1,3]oxazin-2-one (3pb) .

White solid, $87 \mathrm{~h}, 21.8 \mathrm{mg}, 99 \%$ yield, $91 \%$ ee, $\mathrm{mp}$ $172-174{ }^{\circ} \mathrm{C} ;[\alpha]_{\mathrm{D}}{ }^{18}=2.19\left(c: 0.05, \mathrm{CH}_{2} \mathrm{Cl}_{2}\right)$; Determined by HPLC analysis [Daicel chiralpak ODH, $n$-hexane $/ i$-PrOH $=$ $70 / 30,1.0 \mathrm{~mL} / \mathrm{min}, \lambda=254 \mathrm{~nm}, \mathrm{t}$ (minor) $=12.89 \mathrm{~min}, \quad \mathrm{t}$ (major) $=16.43 \mathrm{~min}$.$] ;$

${ }^{1} \mathrm{H}$ NMR $\left(400 \mathrm{MHz}, \mathrm{CDCl}_{3}\right) \delta=7.76-7.68(\mathrm{~m}, 2 \mathrm{H}), 7.49(\mathrm{t}, J=7.6 \mathrm{~Hz}, 1 \mathrm{H}), 7.40-7.28(\mathrm{~m}, 4 \mathrm{H})$, 7.23 (s, 1H), 7.18 (d, $J=10.0 \mathrm{~Hz}, 3 \mathrm{H}), 7.09-7.00(\mathrm{~m}, 2 \mathrm{H}), 5.38$ (dd, $J=8.4,3.6 \mathrm{~Hz}, 1 \mathrm{H}), 3.44$ (qd, $J=$ $17.2,6.0 \mathrm{~Hz}, 2 \mathrm{H})$.

${ }^{13} \mathrm{C}$ NMR $\left(101 \mathrm{MHz}, \mathrm{CDCl}_{3}\right) \delta=196.0,161.7(\mathrm{~d}, J=124.7 \mathrm{~Hz}), 150.4,149.9,136.0,135.8(\mathrm{~d}, J=$ $1.5 \mathrm{~Hz}), 134.0,129.0$ (d, $J=4.5 \mathrm{~Hz}), 129.0,128.8,128.0,127.7$ (d, $J=8.1 \mathrm{~Hz}), 122.5,121.6,119.9$, $116.7(\mathrm{~d}, J=11.6 \mathrm{~Hz}), 116.6,57.9,43.7$.

HRMS (ESI) calcd for [M+Na] ${ }^{+} \mathbf{C}_{22} \mathbf{H}_{15} \mathbf{B r F N O}_{3} \mathbf{N a}^{+}, \mathrm{m} / \mathrm{z}$ : 462.0117,464.0097; observed: 462.0107 464.0096.

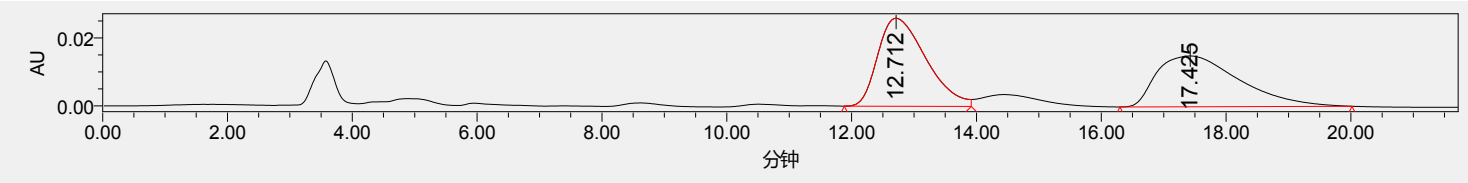

\begin{tabular}{|c|c|c|c|}
\hline & Retention Time & Area & $\%$ Area \\
\hline 1 & 12.712 & 1391812 & 50.32 \\
\hline 2 & 17.425 & 1374258 & 49.68 \\
\hline
\end{tabular}

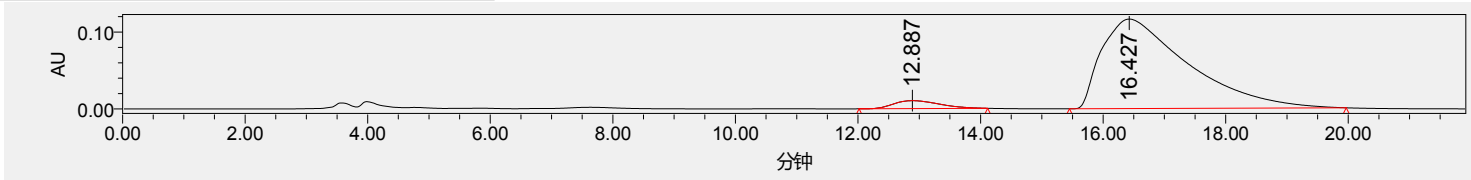

\begin{tabular}{|c|c|c|c|}
\hline & Retention Time & Area & $\%$ Area \\
\hline 1 & 12.887 & 538814 & 4.53 \\
\hline 2 & 16.427 & 11355140 & 95.47 \\
\hline
\end{tabular}<smiles>O=C(C[C@H]1c2cc(Cl)ccc2OC(=O)N1c1ccc(F)cc1)c1ccccc1</smiles>

(S)-6-chloro-3-(4-fluorophenyl)-4-(2-oxo-2-phenylethyl)-3,4-d ihydro-2H-benzo[e][1,3] oxazin-2-one (3qb).

White solid, $98 \mathrm{~h}, 18.6 \mathrm{mg}$, 94\% yield, $90 \%$ ee, mp 120-122 ${ }^{\circ} \mathrm{C} ;[\alpha]_{\mathrm{D}}{ }^{18}=1.44\left(c: 0.07, \mathrm{CH}_{2} \mathrm{Cl}_{2}\right)$; Determined by HPLC analysis [Daicel chiralpak IA, $n$-hexane $/ i$-PrOH $=70 / 30,1.0$ $\mathrm{mL} / \mathrm{min}, \lambda=254 \mathrm{~nm}, \mathrm{t}$ (minor) $=16.73 \mathrm{~min}, \mathrm{t}$ (major) $=25.64$ $\min$,$] ;$ 
${ }^{1} \mathrm{H}$ NMR (400 MHz, $\left.\mathrm{CDCl}_{3}\right) \delta 7.87-7.76(\mathrm{~m}, 2 \mathrm{H}), 7.57(\mathrm{t}, J=7.6 \mathrm{~Hz}, 1 \mathrm{H}), 7.43(\mathrm{dd}, J=14.0,6.8$ $\mathrm{Hz}, 2 \mathrm{H}), 7.40-7.33(\mathrm{~m}, 3 \mathrm{H}), 7.28(\mathrm{~d}, J=2.4 \mathrm{~Hz}, 1 \mathrm{H}), 7.17-7.04(\mathrm{~m}, 3 \mathrm{H}), 5.46$ (dd, $J=8.0,4.0 \mathrm{~Hz}$, $1 \mathrm{H}), 3.61-3.45(\mathrm{~m}, 2 \mathrm{H})$.

${ }^{13} \mathrm{C}$ NMR $\left(101 \mathrm{MHz}, \mathrm{CDCl}_{3}\right) \delta=195.8,161.7(\mathrm{~d}, J=124.7 \mathrm{~Hz}), 150.1,148.4,136.0,135.7,134.0$, 129.74, 129.6, 129.0 (d, $J=4.5 \mathrm{~Hz}), 128.8,128.0,126.2,124.1,118.1,116.7$ (d, $J=11.1 \mathrm{~Hz}), 57.8$, 43.7.

HRMS (ESI) calcd for [M+Na] ${ }^{+} \mathbf{C}_{22} \mathbf{H}_{15} \mathbf{C I F N O} \mathbf{O N a}^{+}$m/z: 418.0622, 420.0593; observed: 418.0612 , 420.0594 .

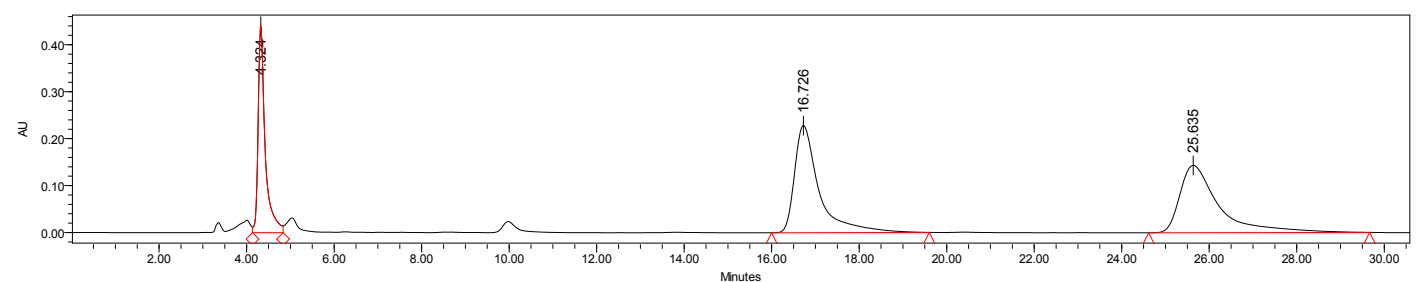

\begin{tabular}{|c|c|c|c|}
\hline & Retention Time & Area & $\%$ Area \\
\hline 1 & 16.726 & 8880973 & 50.09 \\
\hline 2 & 25.635 & 8848584 & 49.91 \\
\hline
\end{tabular}

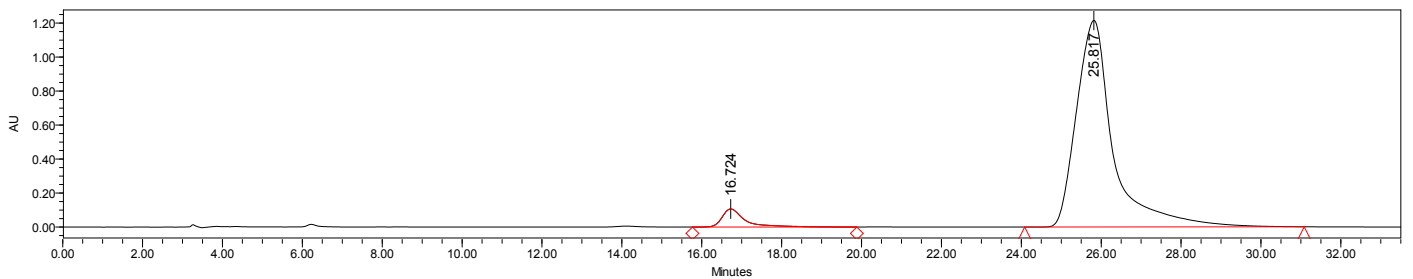

\begin{tabular}{|c|c|c|c|}
\hline & Retention Time & Area & $\%$ Area \\
\hline 1 & 16.724 & 4291227 & 5.24 \\
\hline 2 & 25.817 & 77593607 & 94.76 \\
\hline
\end{tabular}

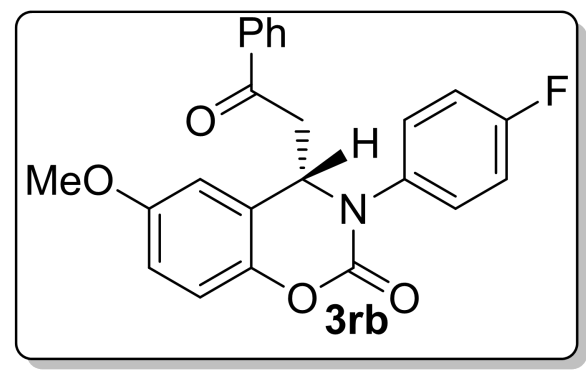

(S)-3-(4-fluorophenyl)-6-methoxy-4-(2-oxo-2-phenylethyl)3,4-dihydro-2H-benzo[e][1,3]oxazin-2-one (3rb).

White solid, $88 \mathrm{~h}, 14.8 \mathrm{mg}, 76 \%$ yield, $91 \%$ ee, $\mathrm{mp}$ $138-140{ }^{\circ} \mathrm{C} ;[\alpha]_{\mathrm{D}}{ }^{18}=1.69\left(c: 0.1, \mathrm{CH}_{2} \mathrm{Cl}_{2}\right)$; Determined by HPLC analysis [Daicel chiralpak IA, $n$-hexane $/ i-\mathrm{PrOH}=$ $70 / 30,1.0 \mathrm{~mL} / \mathrm{min}, \lambda=254 \mathrm{~nm}, \mathrm{t}$ (minor) $=17.41 \mathrm{~min}, \mathrm{t}$ (major) $=29.67 \mathrm{~min}$,$] ;$

${ }^{1} \mathrm{H}$ NMR $\left(400 \mathrm{MHz}, \mathrm{CDCl}_{3}\right) \delta=7.83-7.75(\mathrm{~m}, 2 \mathrm{H}), 7.59-7.51(\mathrm{~m}, 1 \mathrm{H}), 7.46-7.35(\mathrm{~m}, 4 \mathrm{H})$, $7.15-7.06(\mathrm{~m}, 4 \mathrm{H}), 7.02(\mathrm{~d}, J=9.2 \mathrm{~Hz}, 1 \mathrm{H}), 5.46(\mathrm{dd}, J=7.6,4.0 \mathrm{~Hz}, 1 \mathrm{H}), 3.51(\mathrm{qd}, J=17.2,6.0 \mathrm{~Hz}$, $2 \mathrm{H}), 2.28(\mathrm{~s}, 3 \mathrm{H})$. 
${ }^{13} \mathrm{C}$ NMR $\left(101 \mathrm{MHz}, \mathrm{CDCl}_{3}\right) \delta=196.2,161.6(\mathrm{~d}, J=124.2 \mathrm{~Hz}), 150.8,147.7,136.3,136.2(\mathrm{~d}, J=$ $1.5 \mathrm{~Hz}), 134.3,133.8,130.0,129.1(\mathrm{~d}, J=4.5 \mathrm{~Hz}), 128.7,128.0,126.4,122.3,116.6,116.4(\mathrm{~d}, J=$ $3.5 \mathrm{~Hz}), 58.3,44.1,20.8$.

HRMS (ESI) calcd for $[\mathbf{M}+\mathbf{N a}]^{+} \mathbf{C}_{\mathbf{2 3}} \mathbf{H}_{\mathbf{1 8}} \mathbf{F N O}_{4} \mathbf{N a}^{+}, \mathrm{m} / \mathrm{z}$ : 414.1118 , observed: 414.1116 .

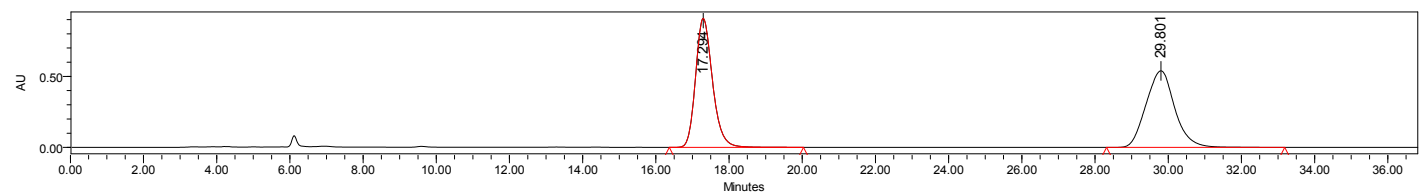

\begin{tabular}{|c|c|c|c|}
\hline & Retention Time & Area & $\%$ Area \\
\hline 1 & 17.294 & 29132763 & 49.78 \\
\hline 2 & 29.801 & 29393927 & 50.22 \\
\hline
\end{tabular}

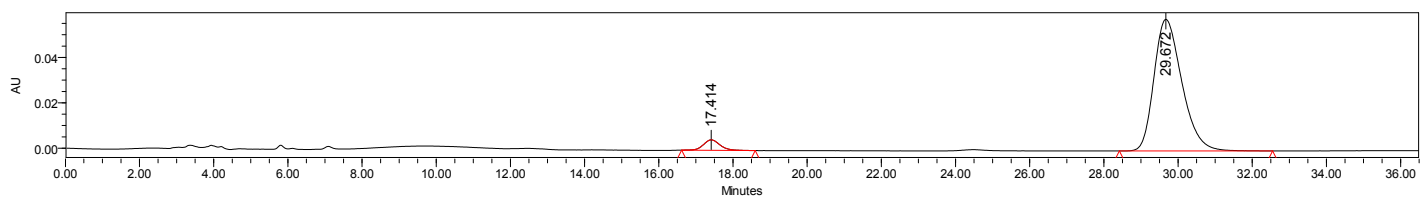

\begin{tabular}{|l|c|c|c|}
\hline & Retention Time & Area & $\%$ Area \\
\hline 1 & 17.414 & 143157 & 4.52 \\
\hline 2 & 29.672 & 3023138 & 95.48 \\
\hline
\end{tabular}

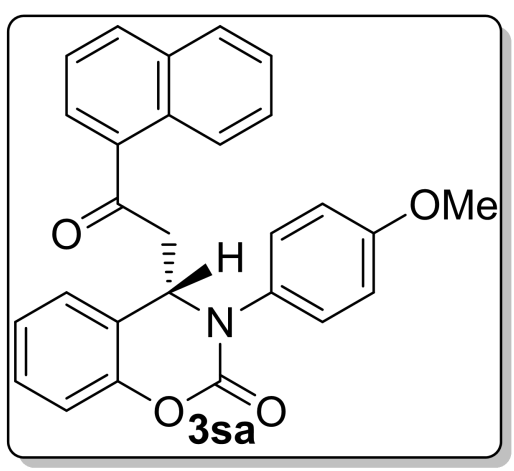

(S)-3-(4-methoxyphenyl)-4-(2-(naphthalen-1-yl)-2-oxoethyl)-3, 4-dihydro-2H-benzo[e][1,3]oxazin-2-one (3sa).

White solid, 87 h, $20.5 \mathrm{mg}, 97 \%$ yield, $85 \%$ ee, mp 148-150 ${ }^{\circ} \mathrm{C} ;[\alpha]_{\mathrm{D}}{ }^{18}=2.13\left(c: 0.1, \mathrm{CH}_{2} \mathrm{Cl}_{2}\right)$; Determined by HPLC analysis [Daicel chiralpak ODH, $n$-hexane $/ i$-PrOH $=80 / 20,1.0$ $\mathrm{mL} / \mathrm{min}, \lambda=254 \mathrm{~nm}, \mathrm{t}$ (minor) $=23.84 \mathrm{~min}, \mathrm{t}($ major $)=26.21$ $\min$,$] .$

${ }^{1} \mathrm{H}$ NMR $\left(400 \mathrm{MHz}, \mathrm{CDCl}_{3}\right) \delta=8.25(\mathrm{~s}, 1 \mathrm{H}), 7.94-7.80$ (m, 4H), 7.57 (dtd, $J=16.4,7.2 \mathrm{~Hz}, 1.2 \mathrm{~Hz}, 2 \mathrm{H}), 7.39-7.32(\mathrm{~m}, 3 \mathrm{H}), 7.29(\mathrm{dd}, J=11.2,4.4 \mathrm{~Hz}, 1 \mathrm{H})$, $7.18-7.11(\mathrm{~m}, 1 \mathrm{H}), 7.08(\mathrm{td}, J=7.6,1.2 \mathrm{~Hz}, 1 \mathrm{H}), 6.97-6.88(\mathrm{~m}, 2 \mathrm{H}), 5.53(\mathrm{dd}, J=8.4,4.0 \mathrm{~Hz}, 1 \mathrm{H})$, $3.80(\mathrm{~s}, 3 \mathrm{H}), 3.67(\mathrm{qd}, J=16.8,6.0 \mathrm{~Hz}, 2 \mathrm{H})$.

${ }^{13} \mathrm{C}$ NMR $\left(101 \mathrm{MHz}, \mathrm{CDCl}_{3}\right) \delta=196.3,158.9,150.8,149.9,135.7,133.7,132.8,132.3,130.0$, $129.6,129.4,128.9,128.6,128.5,127.8,127.0,126.3,124.5,123.4,122.8,116.6,114.9,58.5,55.5$, 44.1 .

HRMS (ESI) calcd for $[\mathbf{M}+\mathbf{N a}]^{+} \mathbf{C}_{\mathbf{2} 7} \mathbf{H}_{21} \mathbf{N O}_{4} \mathbf{N a}^{+}, \mathrm{m} / \mathrm{z}: 446.1368$, observed: 446.1365 . 


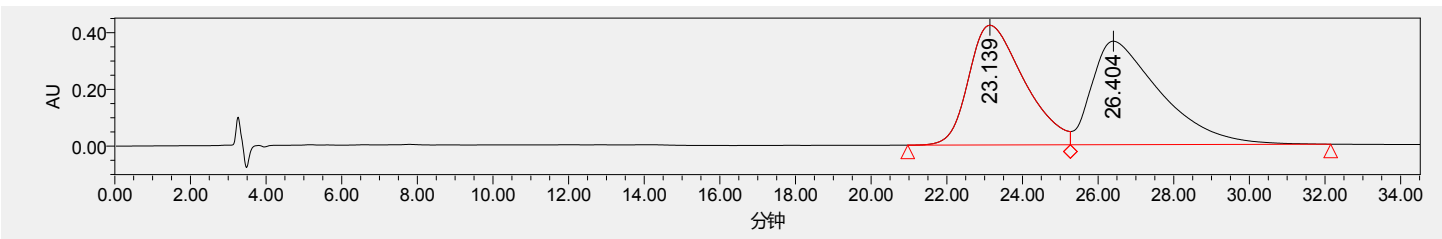

\begin{tabular}{|l|c|c|c|}
\hline & Retention Time & Area & $\%$ Area \\
\hline 1 & 23.139 & 43219012 & 48.43 \\
\hline 2 & 26.404 & 46012950 & 51.57 \\
\hline
\end{tabular}

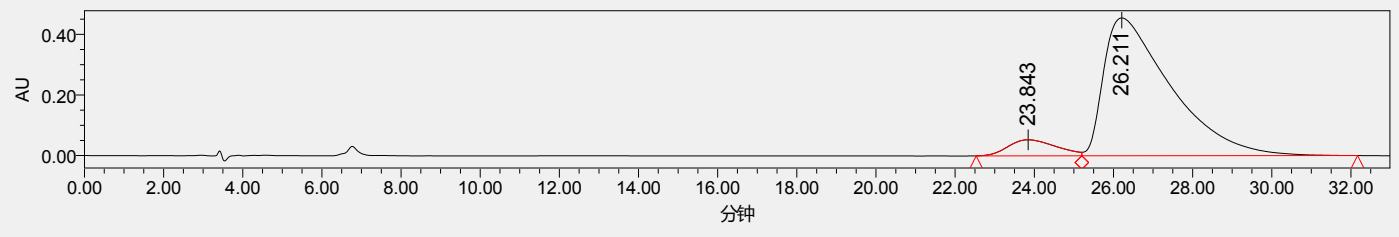

\begin{tabular}{|c|c|c|c|}
\hline & Retention Time & Area & $\%$ Area \\
\hline 1 & 23.843 & 4458875 & 7.69 \\
\hline 2 & 26.211 & 53533727 & 92.31 \\
\hline
\end{tabular}

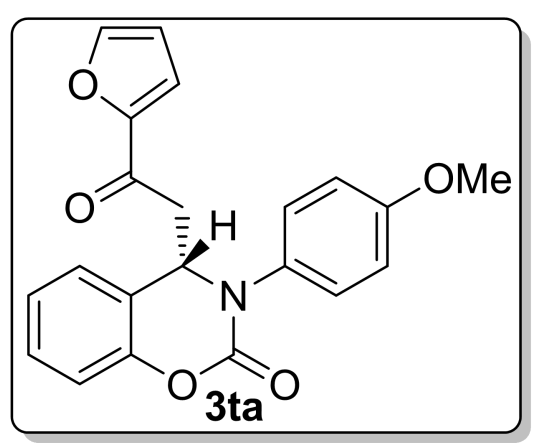

(S)-4-(2-(furan-2-yl)-2-oxoethyl)-3-(4-methoxyphenyl)-3,4-dihy dro-2H-benzo[e][1,3]oxazin-2-one (3ta).

White solid, $61 \mathrm{~h}, 16.7 \mathrm{mg}$, 92\% yield, $85 \%$ ee, mp 202-204 ${ }^{\circ} \mathrm{C} ;[\alpha]_{\mathrm{D}}{ }^{18}=2.08\left(c: 0.09, \mathrm{CH}_{2} \mathrm{Cl}_{2}\right)$; Determined by HPLC analysis [Daicel chiralpak ODH, $n$-hexane $/ i$-PrOH $=80 / 20,1.0$ $\mathrm{mL} / \mathrm{min}, \lambda=254 \mathrm{~nm}, \mathrm{t}$ (minor) $=11.62 \mathrm{~min}, \mathrm{t}$ (major) $=23.05$ $\min$,

${ }^{1} \mathrm{H}$ NMR $\left(400 \mathrm{MHz}, \mathrm{CDCl}_{3}\right) \delta=7.51(\mathrm{~d}, J=0.8 \mathrm{~Hz}, 1 \mathrm{H})$, 7.29 (ddd, $J=13.6,9.6,4.8 \mathrm{~Hz}, 5 \mathrm{H}), 7.16-7.02(\mathrm{~m}, 3 \mathrm{H}), 6.92(\mathrm{dd}, J=7.2,5.2 \mathrm{~Hz}, 2 \mathrm{H}), 6.47$ (dd, $J=$ 3.6, 1.6 Hz, 1H), 5.41 (t, $J=6.4 \mathrm{~Hz}, 1 \mathrm{H}), 3.81(\mathrm{~s}, 3 \mathrm{H}), 3.39$ (d, $J=6.4 \mathrm{~Hz}, 2 \mathrm{H})$.

${ }^{13} \mathrm{C}$ NMR $\left(101 \mathrm{MHz}, \mathrm{CDCl}_{3}\right) \delta=185.1,158.9,152.3,150.7,149.9,146.9,132.7,129.4,128.5$, $126.1,124.4,122.4,117.9,116.6,114.8,112.6,58.3,55.5,44.0$.

HRMS (ESI) calcd for [M+Na] ${ }^{+} \mathbf{C}_{21} \mathbf{H}_{17} \mathbf{N O}_{5} \mathbf{N a}^{+}, \mathrm{m} / \mathrm{z}$ : 386.1004, observed: 386.1001 .

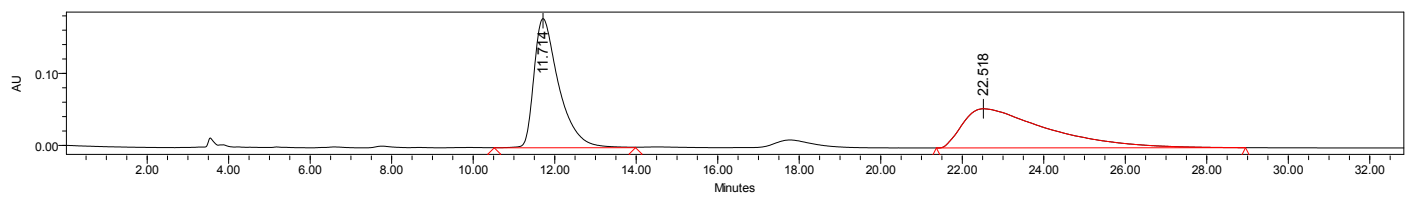

\begin{tabular}{|c|c|c|c|}
\hline & Retention Time & Area & $\%$ Area \\
\hline 1 & 11.714 & 7580652 & 49.23 \\
\hline 2 & 22.518 & 7816880 & 50.77 \\
\hline
\end{tabular}




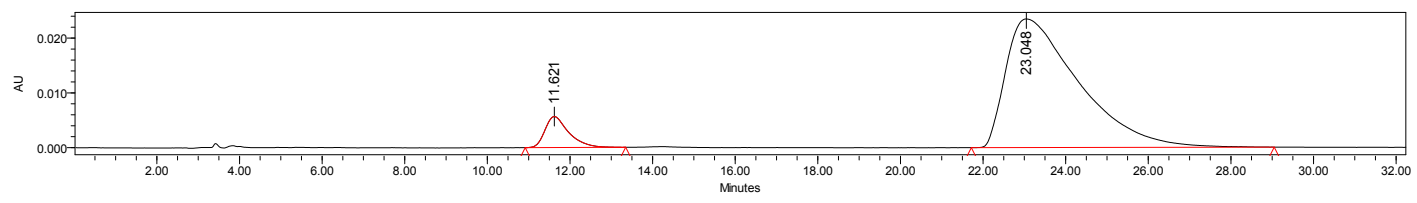

\begin{tabular}{|c|c|c|c|}
\hline & Retention Time & Area & $\%$ Area \\
\hline 1 & 11.621 & 224880 & 7.26 \\
\hline 2 & 23.048 & 2872115 & 92.74 \\
\hline
\end{tabular}

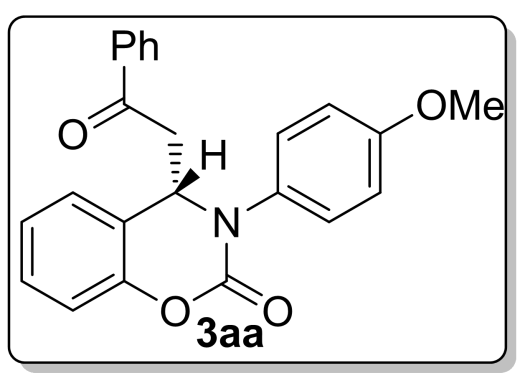

(S)-3-(4-methoxyphenyl)-4-(2-oxo-2-phenylethyl)-3,4-dihydro-2H -benzo[e][1,3]oxazin-2-one (3aa).

White solid, $26 \mathrm{~h}, 18.5 \mathrm{mg}, 99 \%$ yield, $89 \%$ ee, mp $192-194{ }^{\circ} \mathrm{C}$; $[\alpha]_{\mathrm{D}}{ }^{18}=1.27\left(c: 0.3, \mathrm{CH}_{2} \mathrm{Cl}_{2}\right)$; Determined by HPLC analysis [Daicel chiralpak ODH, $n$-hexane $/ i-\mathrm{PrOH}=70 / 30,1.0 \mathrm{~mL} / \mathrm{min}, \lambda$ $=254 \mathrm{~nm}, \mathrm{t}($ minor $)=11.47 \mathrm{~min}, \mathrm{t}$ (major) $=15.79 \mathrm{~min}$.]

${ }^{1} \mathrm{H}$ NMR $\left(400 \mathrm{MHz}, \mathrm{CDCl}_{3}\right) \delta=7.78(\mathrm{dd}, J=8.4,1.2 \mathrm{~Hz}, 2 \mathrm{H})$, $7.57-7.50$ (m, 1H), 7.40 (dd, $J=10.8,4.8 \mathrm{~Hz}, 2 \mathrm{H}), 7.36-7.27$ (m, 4H), 7.10 (ddd, $J=10.8,8.8,4.4$ $\mathrm{Hz}, 2 \mathrm{H}), 6.97-6.89$ (m, 2H), 5.48 (dd, $J=8.4,4.0 \mathrm{~Hz}, 1 \mathrm{H}), 3.81$ (s, 3H), 3.54 (qd, $J=17.2,6.0 \mathrm{~Hz}$, $2 \mathrm{H})$.

${ }^{13} \mathrm{C}$ NMR $\left(101 \mathrm{MHz}, \mathrm{CDCl}_{3}\right) \delta=196.4,158.9,150.8,149.9,136.3,133.7,132.8,129.4,128.7$, 128.5, 128.0, 126.3, 124.4, 122.8, 116.6, 114.9, 58.3, 55.5, 44.0.

HRMS (ESI) calcd for [M+Na] ${ }^{+} \mathbf{C}_{23} \mathbf{H}_{19} \mathbf{N O}_{4} \mathbf{N a}^{+}, \mathrm{m} / \mathrm{z}$ : 396.1212, observed: 396.1214 .

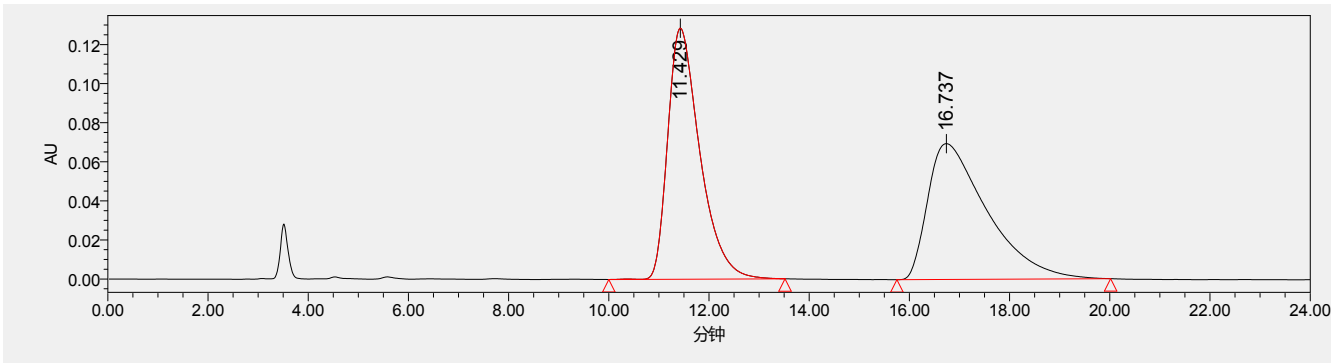

\begin{tabular}{|c|c|c|c|}
\hline & Retention Time & Area & $\%$ Area \\
\hline 1 & 11.429 & 5722883 & 50.02 \\
\hline 2 & 16.737 & 5717397 & 49.98 \\
\hline
\end{tabular}

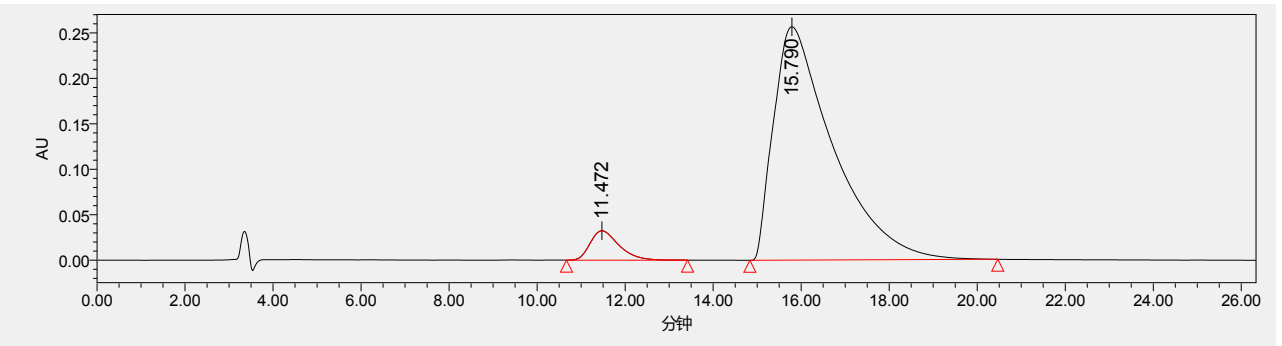




\begin{tabular}{|c|c|c|c|}
\hline & Retention Time & Area & $\%$ Area \\
\hline 1 & 11.472 & 1463781 & 5.73 \\
\hline 2 & 15.790 & 24082569 & 94.27 \\
\hline
\end{tabular}<smiles>O=C(C[C@H]1c2ccccc2OC(=O)N1c1ccccc1F)Oc1ccccc1</smiles>

(S)-3-(2-fluorophenyl)-4-(2-oxo-2-phenylethyl)-3,4-dihydro-2H-benzo[e] [1,3] oxazin-2-one (3ac).

White solid, $89 \mathrm{~h}, 17.5 \mathrm{mg}, 97 \%$ yield, $89 \%$ ee, mp $146-148{ }^{\circ} \mathrm{C}$; $[\alpha]_{\mathrm{D}}{ }^{18}=1.53\left(c: 0.03, \mathrm{CH}_{2} \mathrm{Cl}_{2}\right)$; Determined by HPLC analysis [Daicel chiralpak IA, $n$-hexane $/ i$-PrOH $=70 / 30,0.8 \mathrm{~mL} / \mathrm{min}, \lambda=254 \mathrm{~nm},, \mathrm{t}$ $($ minor $)=14.54 \mathrm{~min}, \mathrm{t}($ major $)=24.22 \mathrm{~min}]$;

${ }^{1} \mathrm{H}$ NMR $\left(400 \mathrm{MHz}, \mathrm{CDCl}_{3}\right) \delta=7.79(\mathrm{~d}, J=7.6 \mathrm{~Hz}, 2 \mathrm{H}), 7.54(\mathrm{t}, J=$ $7.6 \mathrm{~Hz}, 1 \mathrm{H}), 7.47-7.27(\mathrm{~m}, 6 \mathrm{H}), 7.24-7.07(\mathrm{~m}, 4 \mathrm{H}), 5.48(\mathrm{dd}, J=7.2,4.8 \mathrm{~Hz}, 1 \mathrm{H}), 3.62-3.48(\mathrm{~m}$, $2 \mathrm{H})$.

${ }^{13} \mathrm{C}$ NMR $\left(101 \mathrm{MHz}, \mathrm{CDCl}_{3}\right) \delta=196.2,158.2(\mathrm{~d}, J=125.7), 150.0,149.8,136.2,133.7,130.2(\mathrm{~d}, J$ $=4.0 \mathrm{~Hz}), 130.0,129.4,128.7,128.0,127.5$ (d, $J=6.6 \mathrm{~Hz}), 126.3,125.1$ (d, $J=1.5 \mathrm{~Hz}), 124.7,122.6$, $117.1(\mathrm{~d}, J=9.6 \mathrm{~Hz}), 116.7,57.8,44.5$.

HRMS (ESI) calcd for [M+Na] ${ }^{+} \mathbf{C}_{22} \mathbf{H}_{16} \mathbf{F N O}_{3} \mathbf{N a}^{+}, \mathrm{m} / \mathrm{z}$ : 384.1012, observed: 384.1008 .

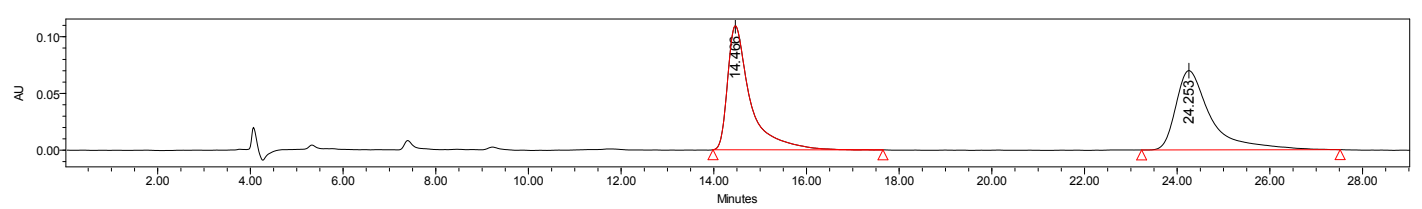

\begin{tabular}{|c|c|c|c|}
\hline & Retention Time & Area & $\%$ Area \\
\hline 1 & 14.466 & 3724059 & 50.48 \\
\hline 2 & 24.253 & 3653835 & 49.52 \\
\hline
\end{tabular}

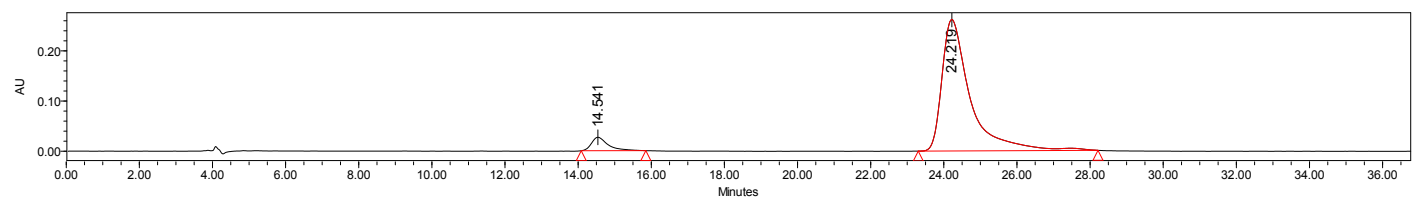

\begin{tabular}{|c|c|c|c|}
\hline & Retention Time & Area & $\%$ Area \\
\hline 1 & 14.541 & 845642 & 5.67 \\
\hline 2 & 24.219 & 14059403 & 94.33 \\
\hline
\end{tabular}<smiles>O=C(/C=C/c1ccccc1OC(=O)N[Cl+3])Oc1ccccc1C(=O)O</smiles>

4ab

${ }^{1} \mathrm{H}$ NMR $\left(400 \mathrm{MHz}, \mathrm{CDCl}_{3}\right) \delta=7.99(\mathrm{t}, J=11.2 \mathrm{~Hz}, 3 \mathrm{H}), 7.76(\mathrm{~d}, J=7.6$ $\mathrm{Hz}, 1 \mathrm{H}), 7.54(\mathrm{dd}, J=14.8,6.4 \mathrm{~Hz}, 3 \mathrm{H}), 7.43(\mathrm{dd}, J=12.8,6.4 \mathrm{~Hz}, 5 \mathrm{H})$, $7.28(\mathrm{t}, J=7.6 \mathrm{~Hz}, 2 \mathrm{H}), 6.99$ (t, $J=8.4 \mathrm{~Hz}, 2 \mathrm{H})$. 
${ }^{13} \mathrm{C} \mathrm{NMR}\left(101 \mathrm{MHz}, \mathrm{CDCl}_{3}\right) \delta=190.6,159.3(\mathrm{~d}, J=122.2 \mathrm{~Hz}), 151.4,149.5,138.2,137.9,133.2$, 133.0, 131.4, $128.6(\mathrm{~d}, J=3.0 \mathrm{~Hz}), 127.5,126.3,123.8,123.4,120.6,115.8(\mathrm{~d}, J=11.1 \mathrm{~Hz})$.

HRMS (ESI) calcd for [M+Na $]^{+} \mathbf{C}_{22} \mathbf{H}_{16} \mathbf{F N O}_{3} \mathbf{N a}^{+}, \mathrm{m} / \mathrm{z}$ : 384.1012, observed: 384.1003 .

\section{Gram scale experiment}<smiles>O=C(/C=C/c1ccccc1O)c1ccccc1</smiles>

1a

$2.93 \mathrm{mmol}$ $0.66 \mathrm{~g}$<smiles>O=C=Nc1ccc(F)cc1</smiles>

$2 \mathrm{~b}$

$4.39 \mathrm{mmol}$

$527 \mu \mathrm{L}$
BG-3.1.3HBArF ${ }_{4}$

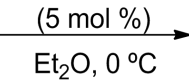

$\mathrm{Et}_{2} \mathrm{O}, 0^{\circ} \mathrm{C}$<smiles>O=C(C[C@H]1c2ccccc2OC(=O)N1c1ccc(F)cc1)c1ccccc1</smiles>

$99 \%$ yield, $94 \%$ ee

$1.05 \mathrm{~g}$

In a round bottomed flask with a magnetic stirring barguanidinium salt BG-3 $\cdot 1.3 \mathrm{HBAr}^{\mathrm{F}} 4(5 \mathrm{~mol} \%)$ and 2-hydroxyphenyl-substituted enones $1 \mathbf{a}(2.93 \mathrm{mmol})$ in ether $(90 \mathrm{~mL})$ were stirred at $30^{\circ} \mathrm{C}$ for 30 $\min$. Then the round-bottomed flask was transformed to $0{ }^{\circ} \mathrm{C}$ and isocyanate $2 \mathbf{b}(3.0 \mathrm{mmol})$ was added in one-portion at $0{ }^{\circ} \mathrm{C}$. The mixture was stirred at $0{ }^{\circ} \mathrm{C}$, and was detected by TLC. After completion, flash column chromatography provided the desired product (pet/EtOAc $=3 / 1$ as eluent) with almost quantitative yield. The product was directed for HPLC and NMR analysis.

\section{X-ray crystal structure of the product 3ab}

Identification code

Empirical formula

Formula weight

Temperature/K

Crystal system

Space group

$\mathrm{a} / \AA$

$\mathrm{b} / \AA ̊$

$\mathrm{c} / \AA$

$\alpha /^{\circ}$ fxm-cc-1

$\mathrm{C}_{24} \mathrm{H}_{19} \mathrm{FN}_{2} \mathrm{O}_{3}$

402.41

293

orthorhombic

$\mathrm{P} 2{ }_{1}{ }_{1} 2_{1}$

$5.86821(12)$

16.6995(3)

20.8172(5)

90 
$\begin{array}{ll}\beta /{ }^{\circ} & 90 \\ \gamma /{ }^{\circ} & 90\end{array}$

Volume $/ \AA^{3} \quad 2040.01(8)$

Z 4

$\rho_{\text {calc } g / \mathrm{cm}^{3}} \quad 1.310$

$\mu / \mathrm{mm}^{-1} \quad 0.770$

$\mathrm{F}(000) \quad 840.0$

Crystal size $/ \mathrm{mm}^{3} \quad 0.7 \times 0.3 \times 0.2$

Radiation $\quad \operatorname{CuK} \alpha(\lambda=1.54184)$

$2 \Theta$ range for data collection $/{ }^{\circ} 10.014$ to 144.388

Index ranges $\quad-7 \leq \mathrm{h} \leq 4,-20 \leq \mathrm{k} \leq 19,-25 \leq 1 \leq 23$

Reflections collected $\quad 11324$

Independent reflections $\quad 3961\left[\mathrm{R}_{\mathrm{int}}=0.0396, \mathrm{R}_{\text {sigma }}=0.0301\right]$

Data/restraints/parameters $\quad 3961 / 0 / 272$

Goodness-of-fit on $\mathrm{F}^{2} \quad 1.039$

Final $R$ indexes $[\mathrm{I}>=2 \sigma(\mathrm{I})] \quad \mathrm{R}_{1}=0.0503, \mathrm{wR}_{2}=0.1358$

Final $\mathrm{R}$ indexes [all data] $\quad \mathrm{R}_{1}=0.0553, \mathrm{wR}_{2}=0.1434$

Largest diff. peak/hole / e $\AA^{-3} 0.21 /-0.23$

Flack parameter $\quad-0.07(13)$ 


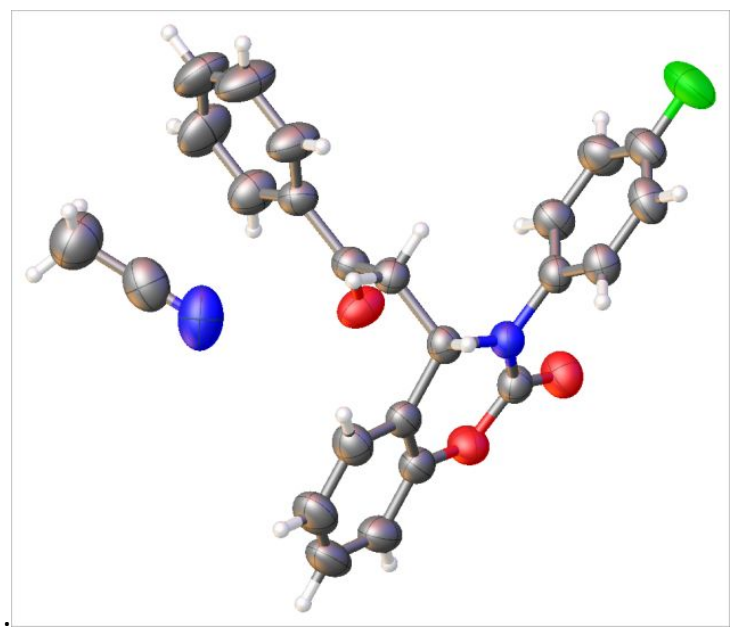

\section{The mechanism of cascade aza-Michael reaction}

(1)

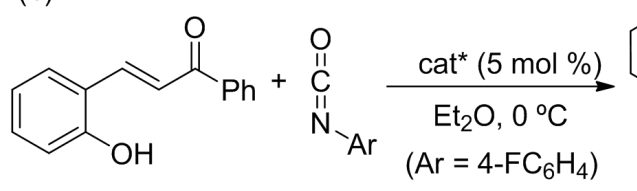

1a

2b<smiles>O=C(N[Al])Oc1ccccc1/C=C/C(=O)c1ccccc1</smiles>

4ab

4ab: $99 \%$ yield;

4ab: none

4ab: $99 \%$ yield;<smiles>O=C(O)C[C@H]1c2ccccc2OC(=O)N1[Al]</smiles>

$3 a b$

conditions:

a. cat $^{*}=$ BG-3: $51 \mathrm{~h}$

b. cat $^{*}=\mathrm{BG}-3 \cdot 2 \mathrm{HBAr}_{4} ; 48 \mathrm{~h}$

c. cat $^{*}=\mathrm{Et}_{3} \mathrm{~N}(100 \mathrm{~mol} \%) ; 51 \mathrm{~h}$

(2)

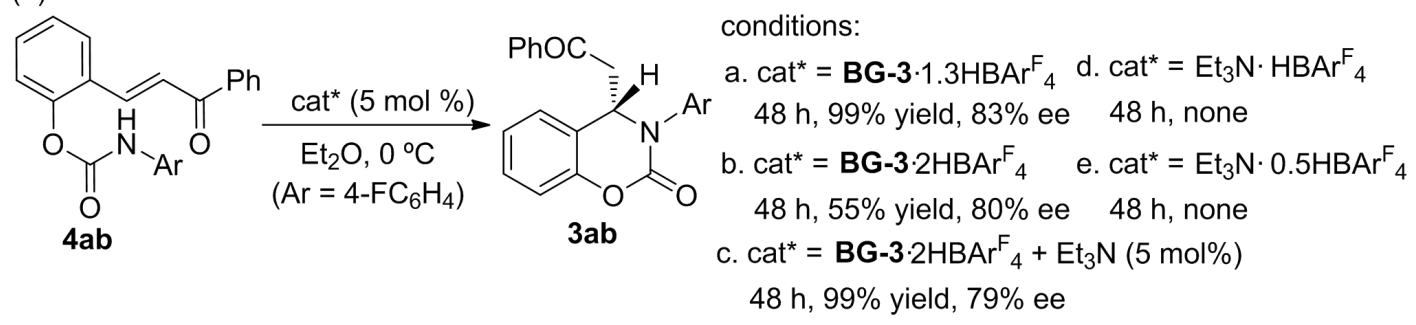

Typical procedure for eq 1a: In a test tube with a magnetic stirring bar, bisguanidine BG-3 (5 mol \%) and 2-hydroxyphenyl-substituted enones 1a $(0.05 \mathrm{mmol})$ in ether $(1.5 \mathrm{~mL})$ were stirred at 30 ${ }^{\circ} \mathrm{C}$ for $30 \mathrm{~min}$, Then the test tube was transformed to $0{ }^{\circ} \mathrm{C}$ and the isocyanate $\mathbf{2 b}(0.075 \mathrm{mmol})$ was added in one-portion. The mixture was stirred at $0{ }^{\circ} \mathrm{C}$ for $51 \mathrm{~h}$. Flash column chromatography provided the $\mathbf{4 a b}$ with almost quantitative yield.

Typical procedure for eq 1b: In a test tube with a magnetic stirring bar, bisguanidinium salt BG-3 $2 \mathrm{HBAr}_{4}{ }_{4}(5 \mathrm{~mol} \%)$ and 2-hydroxyphenyl-substituted enones 1a $(0.05 \mathrm{mmol})$ in ether $(1.5 \mathrm{~mL})$ were stirred at $30{ }^{\circ} \mathrm{C}$ for $30 \mathrm{~min}$, Then the test tube was transformed to $0{ }^{\circ} \mathrm{C}$ and the isocyanate $\mathbf{2 b}$ $(0.075 \mathrm{mmol})$ was added in one-portion. The mixture was stirred at $0{ }^{\circ} \mathrm{C}$ for $48 \mathrm{~h}$. No product was detected. 
Typical procedure for eq $1 \mathrm{c}$ : In a test tube with a magnetic stirring bar, 2-hydroxyphenyl-substituted enones $1 \mathrm{a}(0.05 \mathrm{mmol})$ and $\mathrm{Et}_{3} \mathrm{~N}(100 \mathrm{~mol} \%)$ in ether $(1.5 \mathrm{~mL})$ were stirred at $30^{\circ} \mathrm{C}$ for $30 \mathrm{~min}$, Then the test tube was transformed to $0{ }^{\circ} \mathrm{C}$ and the isocyanate $2 \mathbf{b}(0.075 \mathrm{mmol})$ was added in one-portion. The mixture was stirred at $0{ }^{\circ} \mathrm{C}$ for $51 \mathrm{~h}$. Flash column chromatography provided the 4ab with almost quantitative yield.

Typical procedure for eq 2a: In a test tube with a magnetic stirring bar, bisguanidinium BG-3 $1.3 \mathrm{HBAr}^{\mathrm{F}}{ }_{4}(5 \mathrm{~mol} \%)$ and $4 \mathbf{a b}(18.05 \mathrm{mg}, 0.05 \mathrm{mmol})$ in ether $(1.5 \mathrm{~mL})$ were stirred at $0{ }^{\circ} \mathrm{C}$ for $48 \mathrm{~h}$. After completion, flash column chromatography provided the product with almost quantitative yield.

Typical procedure for eq $2 \mathrm{~b}$ : In a test tube with a magnetic stirring bar, bisguanidinium salt BG-3 $2 \mathrm{HBAr}^{\mathrm{F}} 4(5 \mathrm{~mol} \%)$ and $4 \mathbf{a b}(18.05 \mathrm{mg}, 0.05 \mathrm{mmol})$ in ether $(1.5 \mathrm{~mL})$ were stirred at $0{ }^{\circ} \mathrm{C}$ and detected by TLC. After $48 \mathrm{~h}$, flash column chromatography provided the product with $55 \%$ yield, $80 \%$ ee.

Typical procedure for eq $2 \mathrm{c}$ : In a test tube with a magnetic stirring bar, bisguanidinium salt BG-3.2HBArF $\mathrm{F}_{4}(5 \mathrm{~mol} \%), \mathrm{Et}_{3} \mathrm{~N}(5 \mathrm{~mol} \%)$ and $4 \mathbf{a b}(18.05 \mathrm{mg}, 0.05 \mathrm{mmol})$ in ether $(1.5 \mathrm{~mL})$ were stirred at $0{ }^{\circ} \mathrm{C}$ and detected by TLC. After $48 \mathrm{~h}$, flash column chromatography provided the product with $99 \%$ yield, $79 \%$ ee.

Typical procedure for eq $2 \mathrm{~d}$ : In a test tube with a magnetic stirring bar, bisguanidinium salt $\mathrm{Et}_{3} \mathrm{~N} \cdot \mathrm{HBAr}^{\mathrm{F}}{ }_{4}(5 \mathrm{~mol} \%)$ and $\mathbf{4 a b}(18.05 \mathrm{mg}, 0.05 \mathrm{mmol})$ in ether $(1.5 \mathrm{~mL})$ were stirred at $0{ }^{\circ} \mathrm{C}$ and detected by TLC. After $48 \mathrm{~h}$, No product was detected.

Typical procedure for eq 2e: In a test tube with a magnetic stirring bar, bisguanidinium salt $\mathrm{Et}_{3} \mathrm{~N} \cdot 0.5 \mathrm{HBAr}_{4}^{\mathrm{F}}(5 \mathrm{~mol} \%)$ and $4 \mathbf{a b}(18.05 \mathrm{mg}, 0.05 \mathrm{mmol})$ in ether $(1.5 \mathrm{~mL})$ were stirred at $0{ }^{\circ} \mathrm{C}$ and detected by TLC. After 48 h, No product was detected.

\section{NMR spectra}




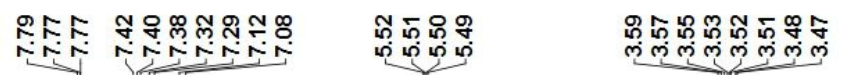
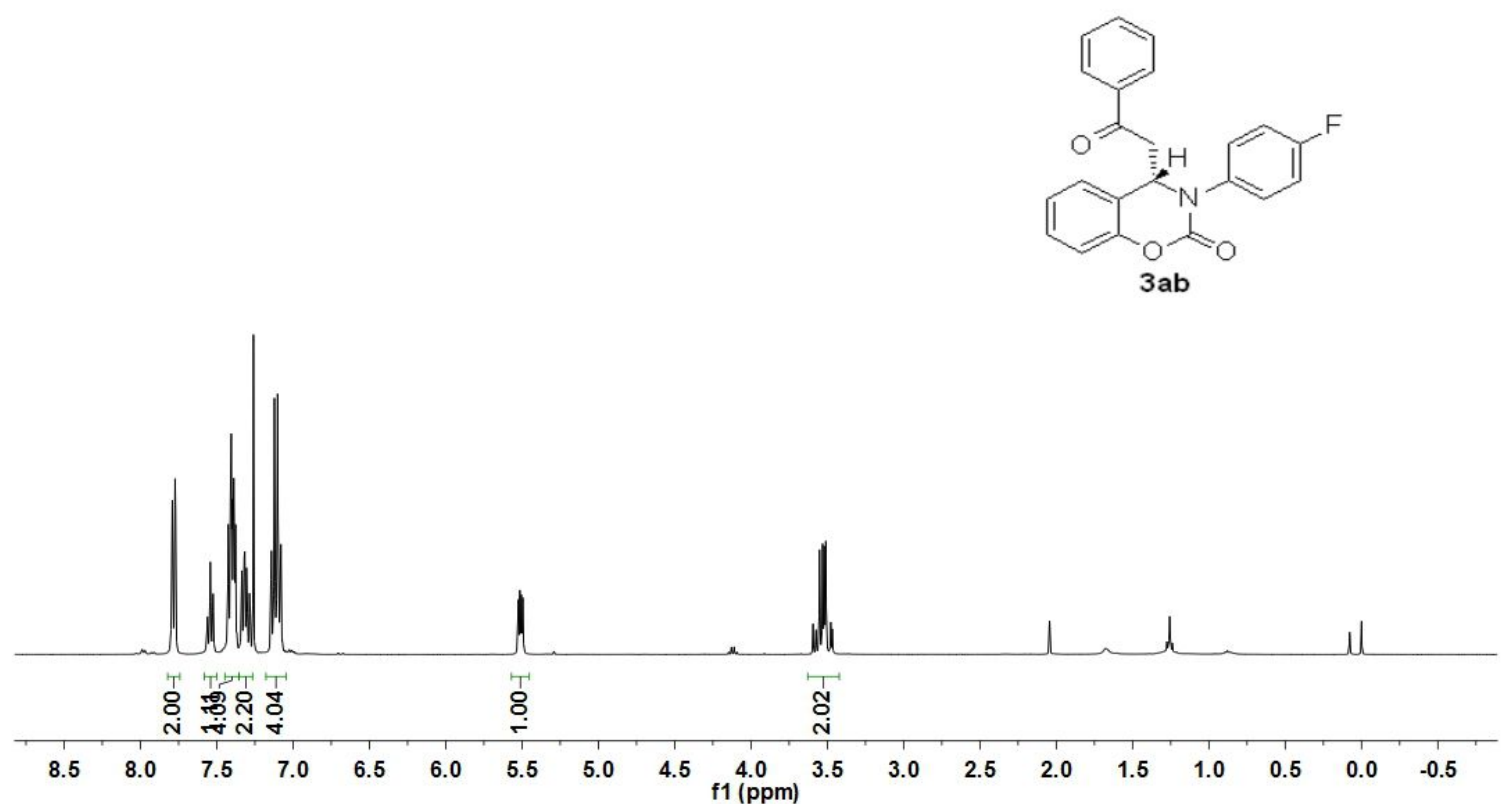

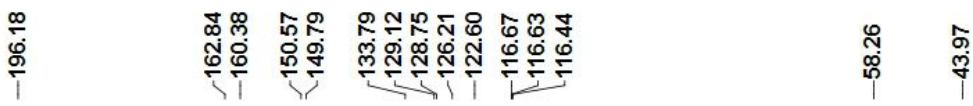

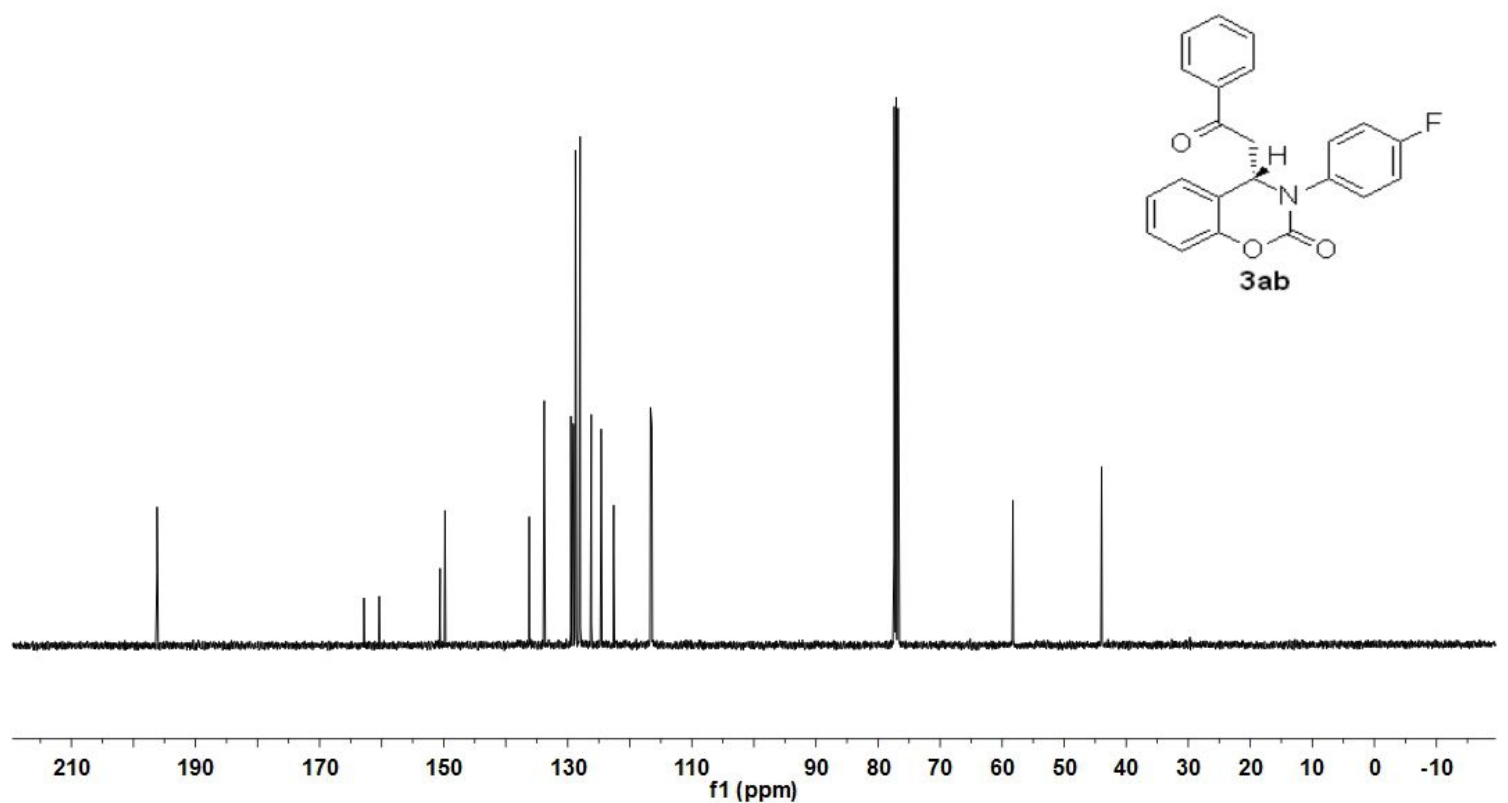




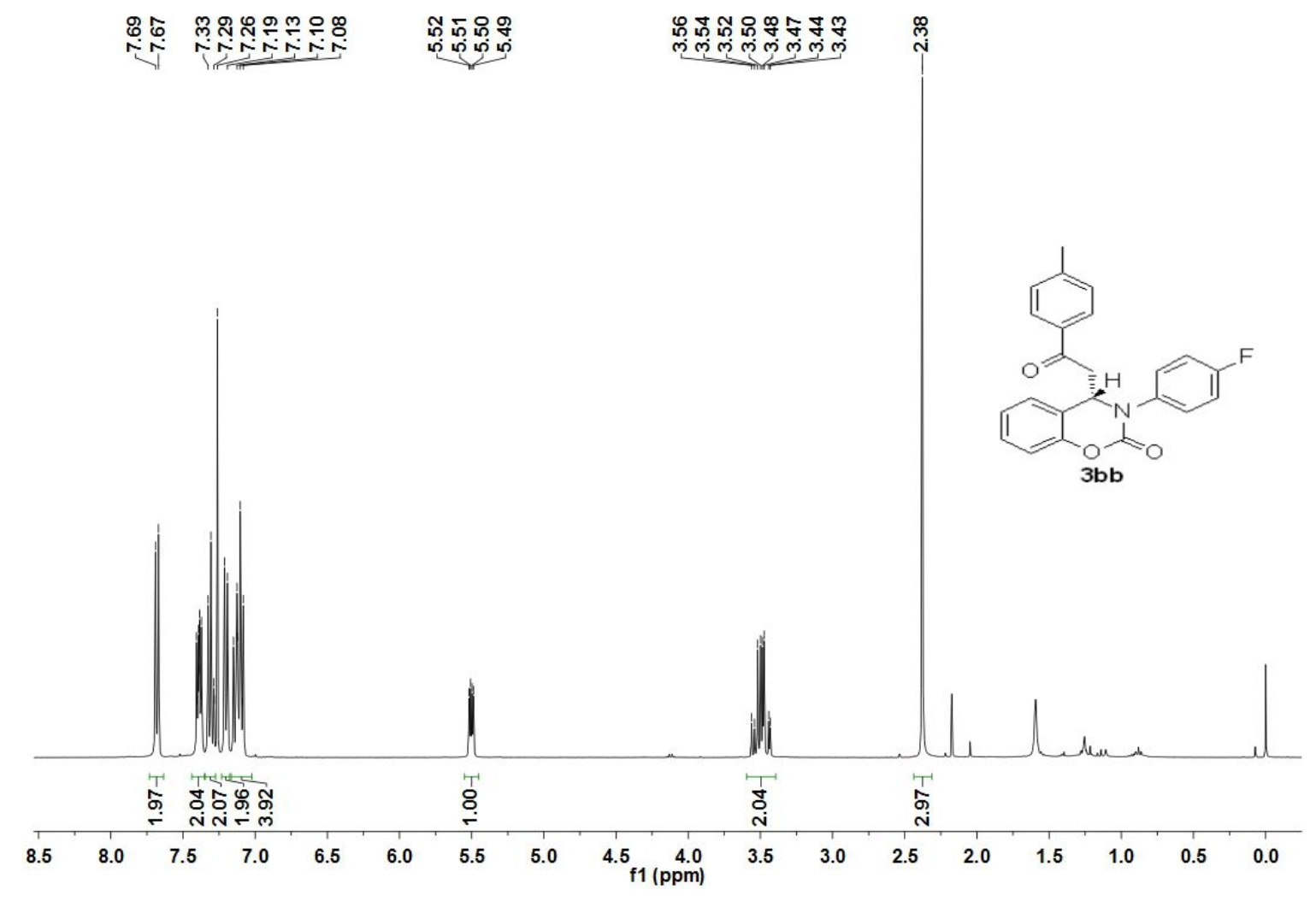

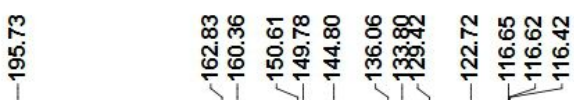

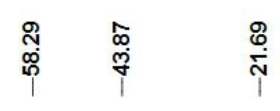

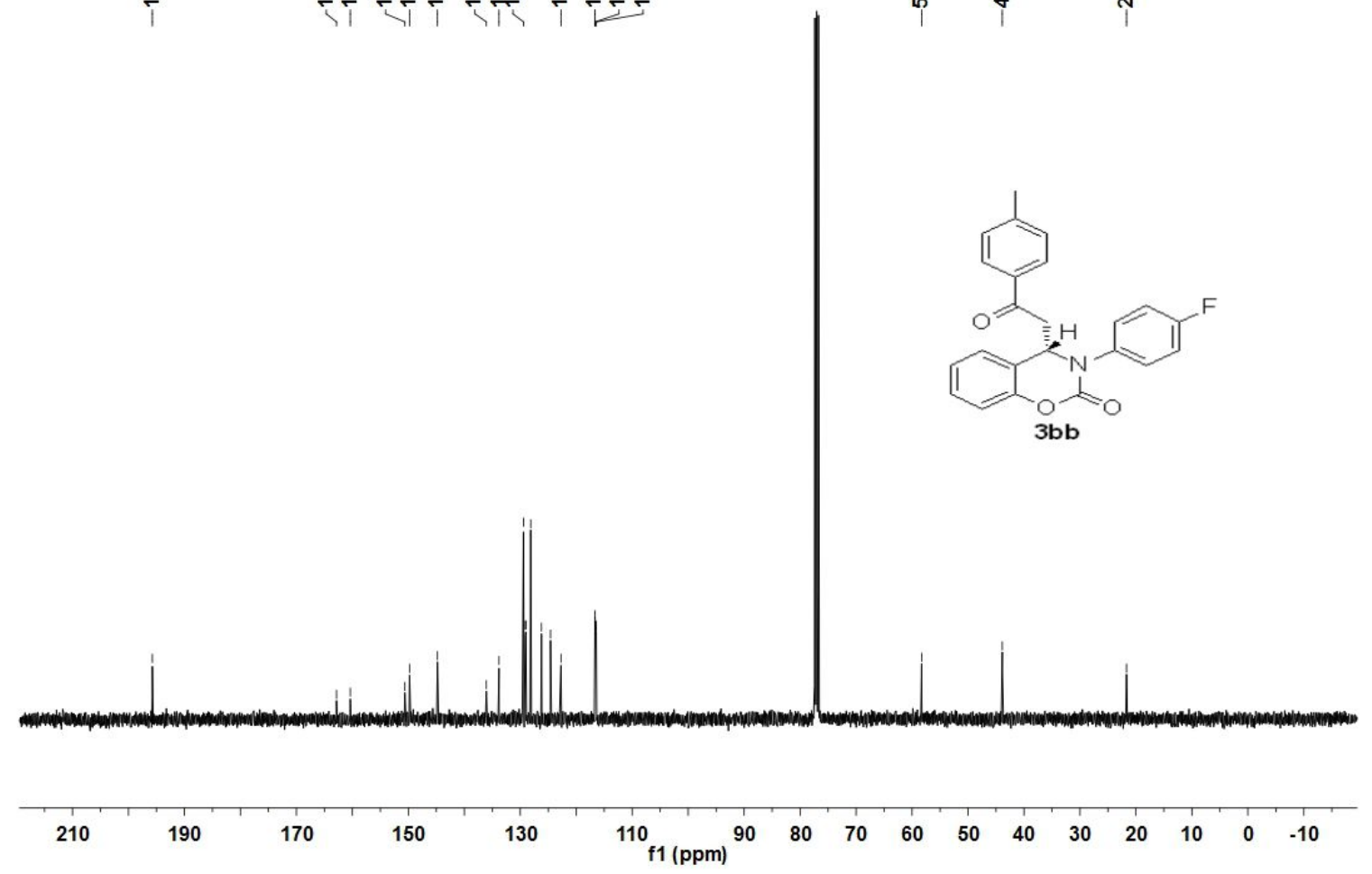




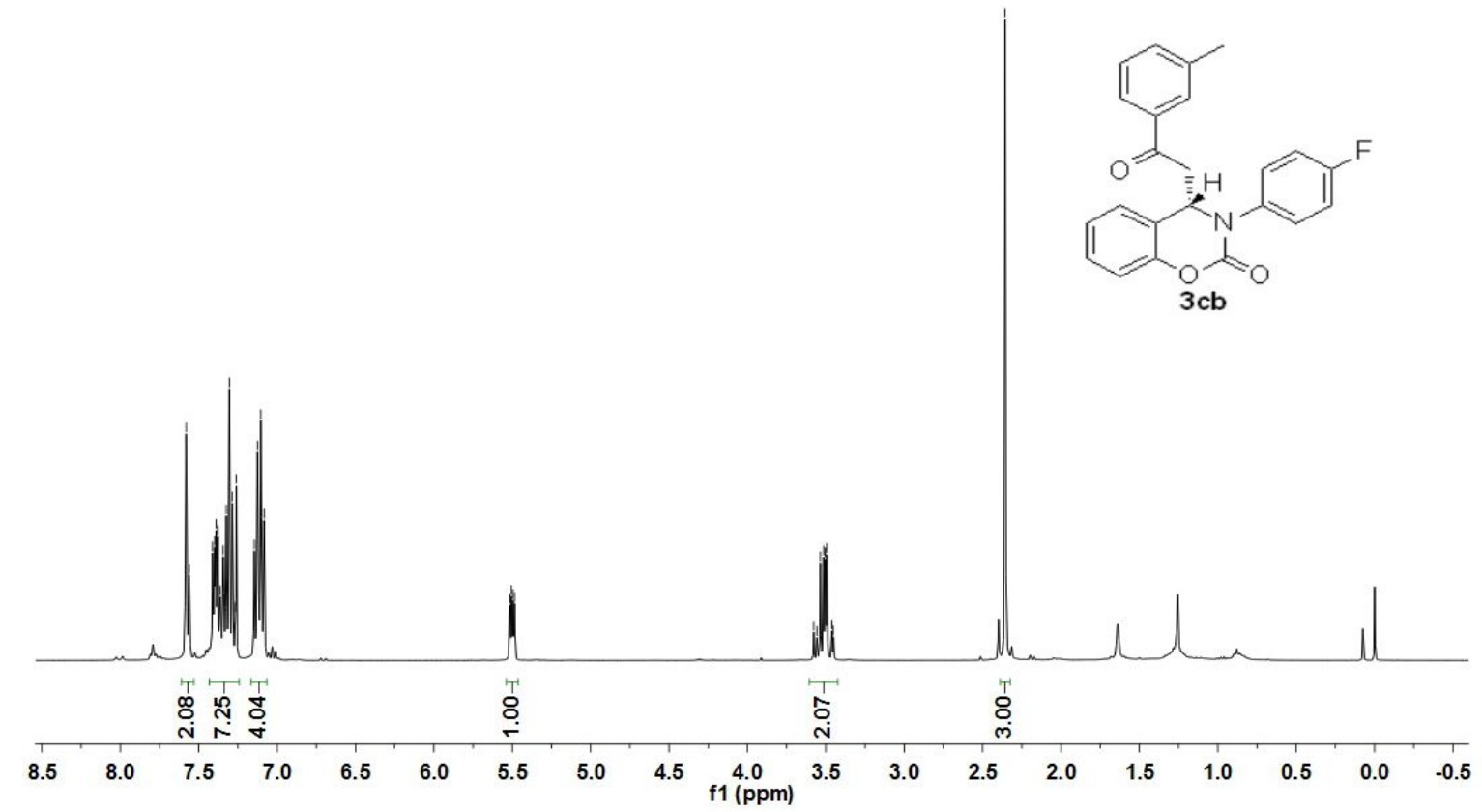

V)

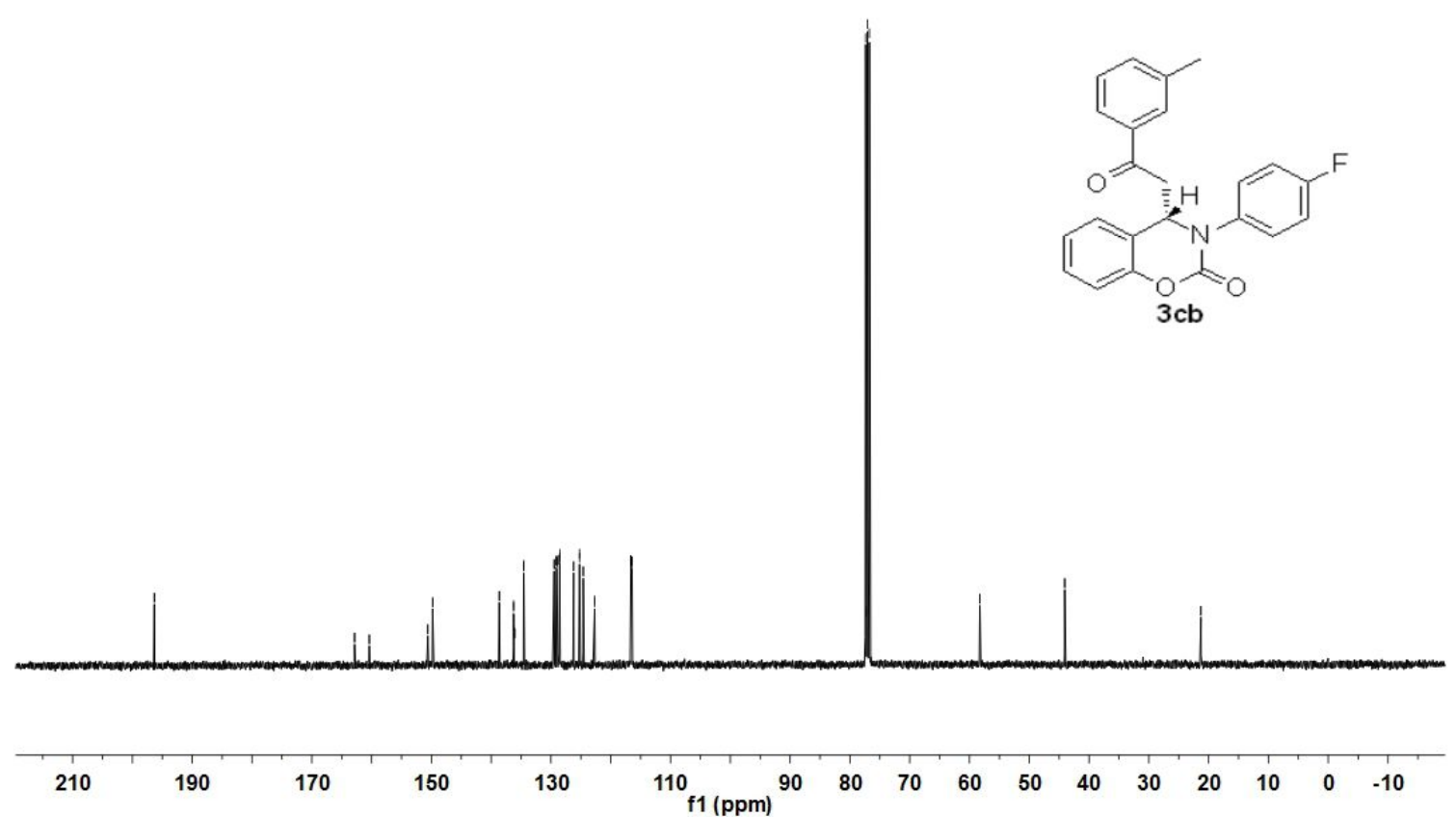




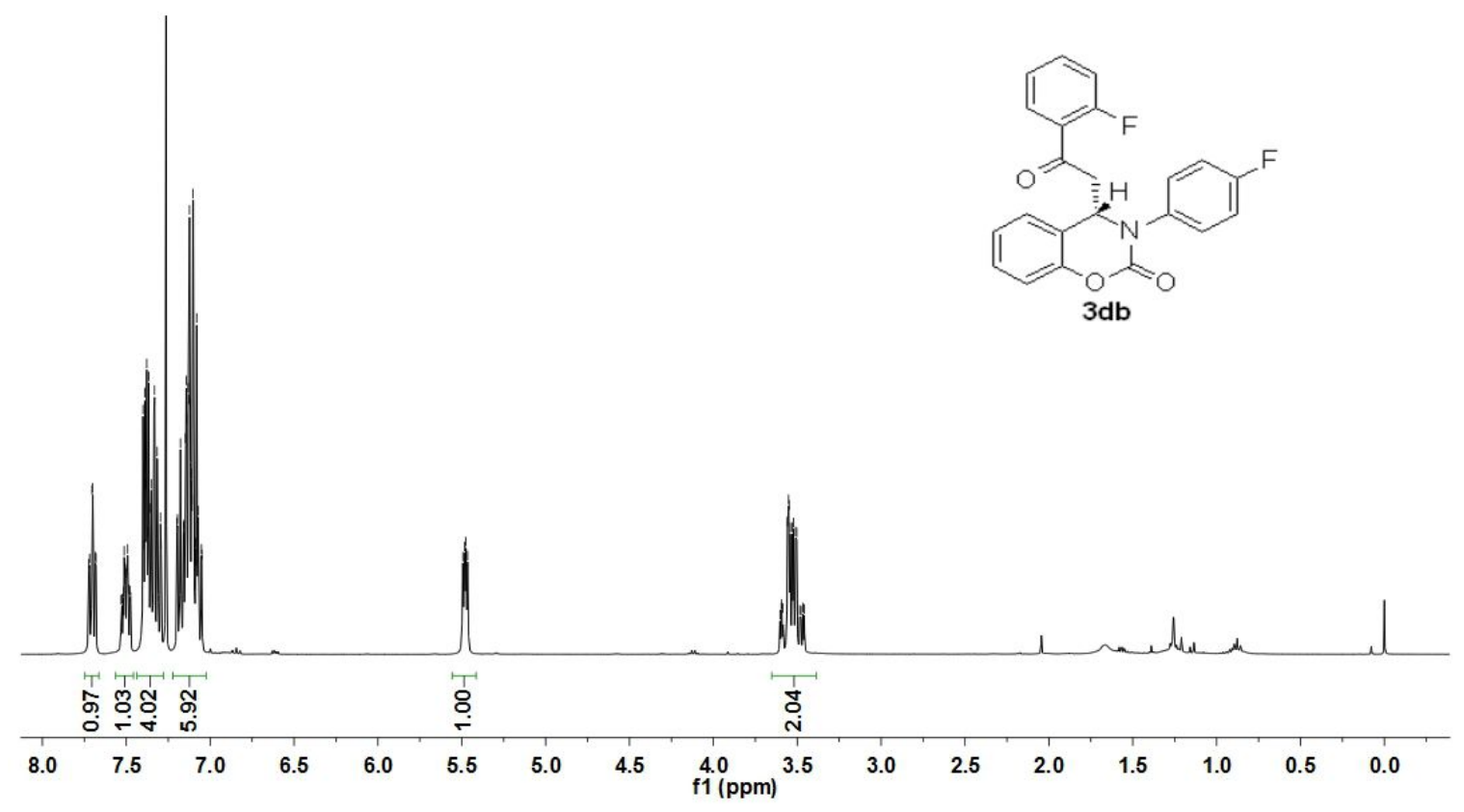

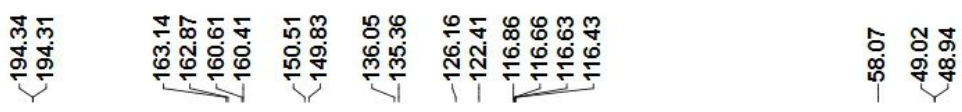

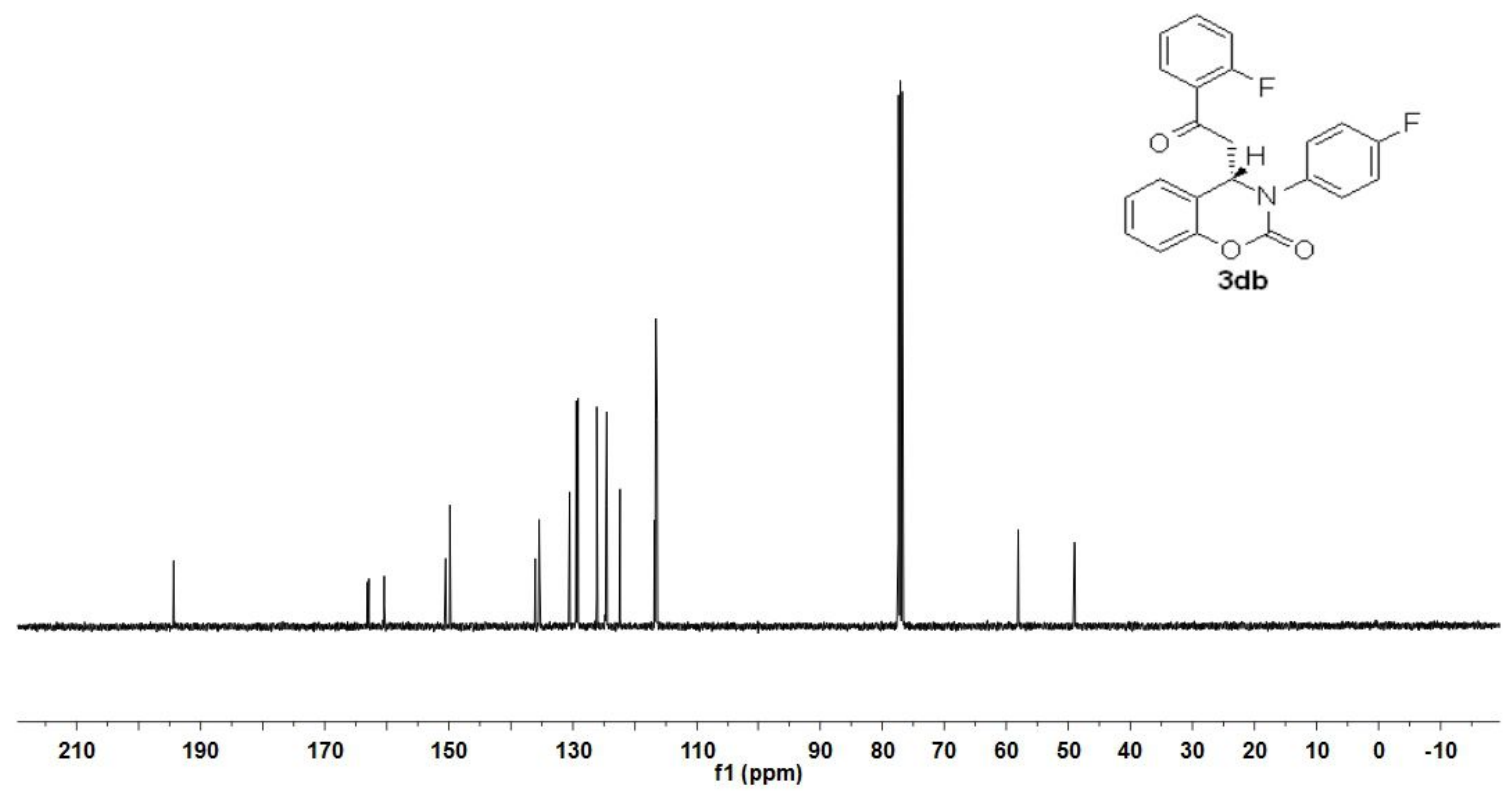



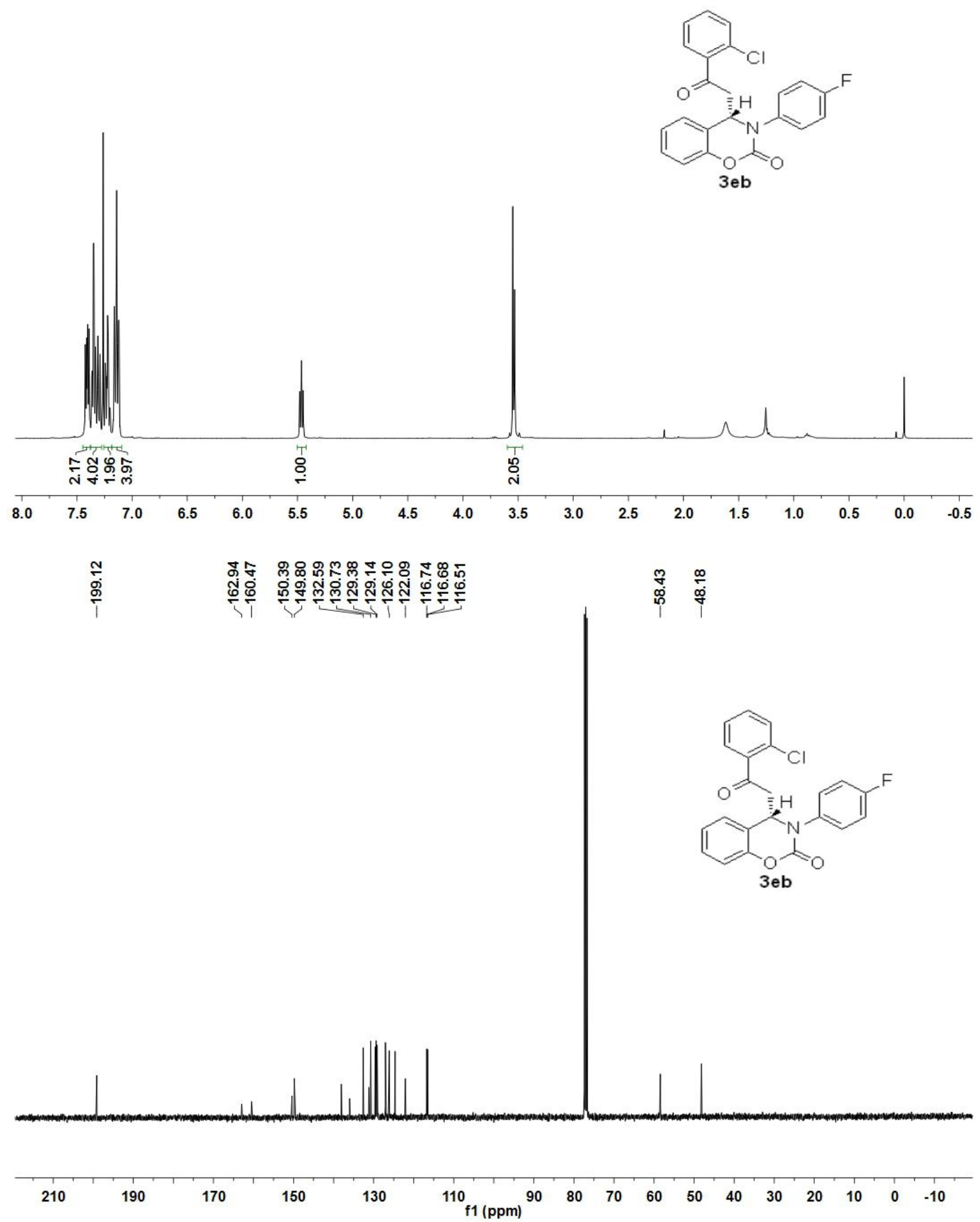

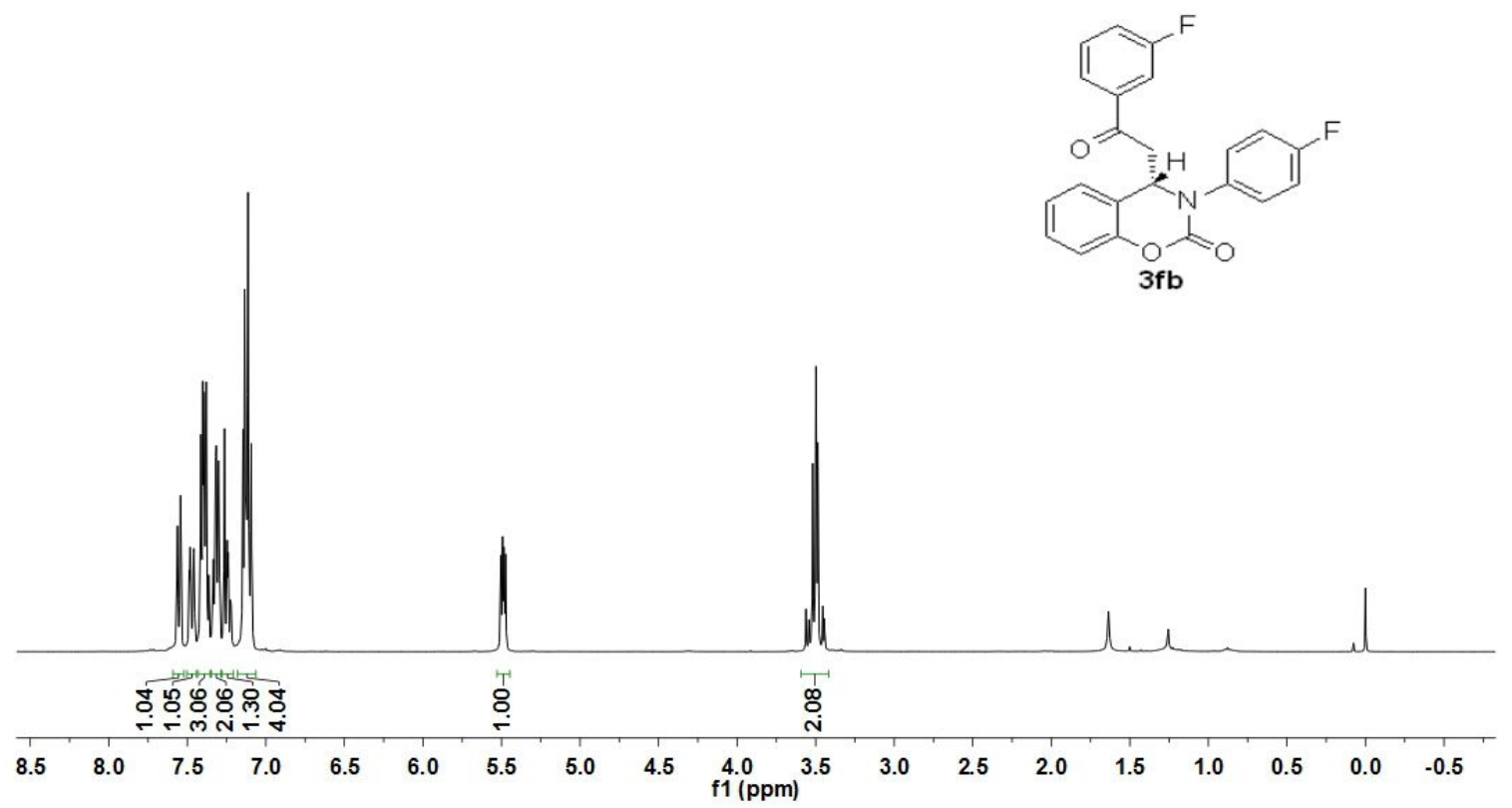

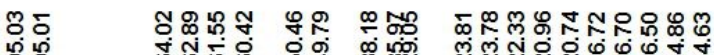

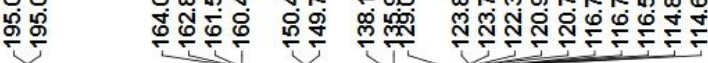
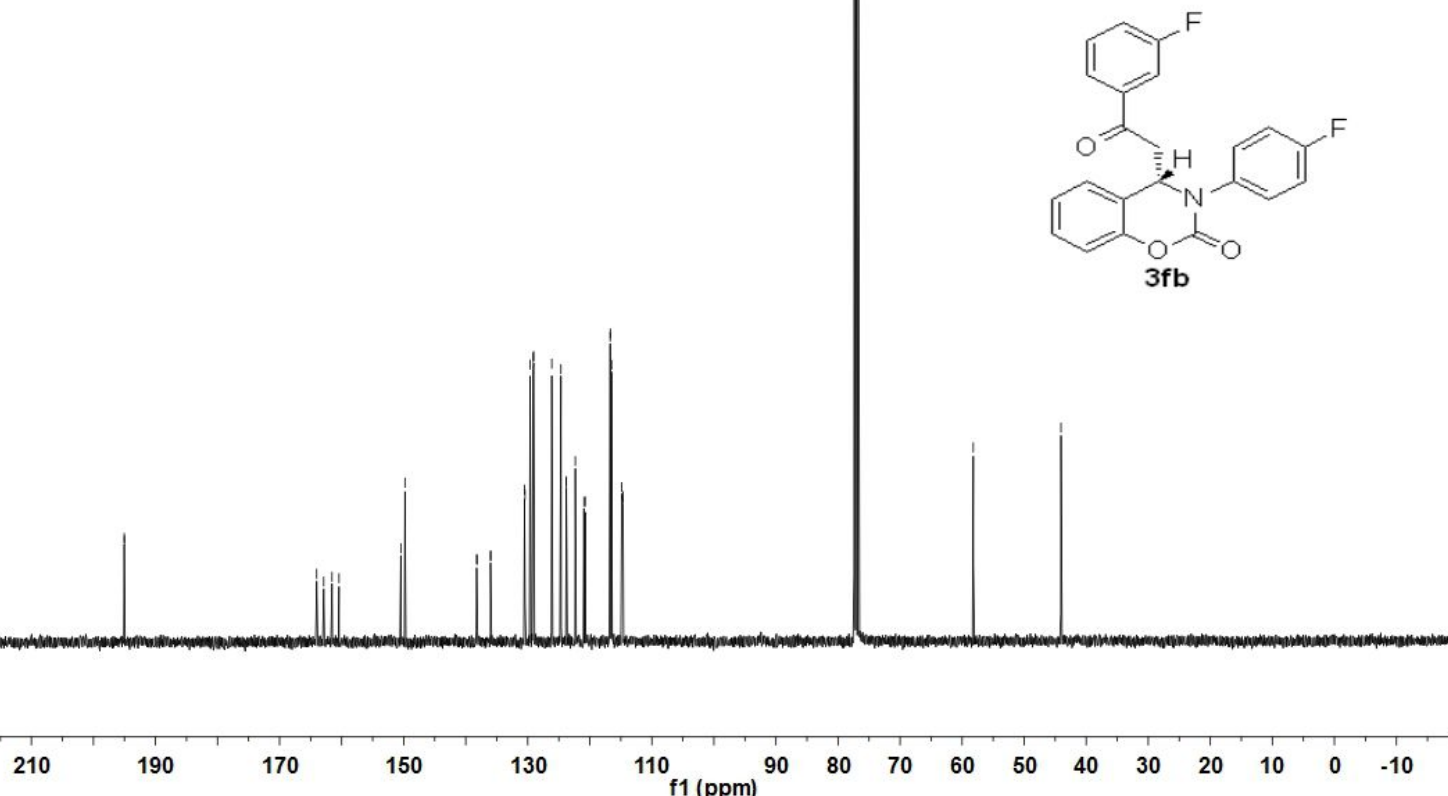

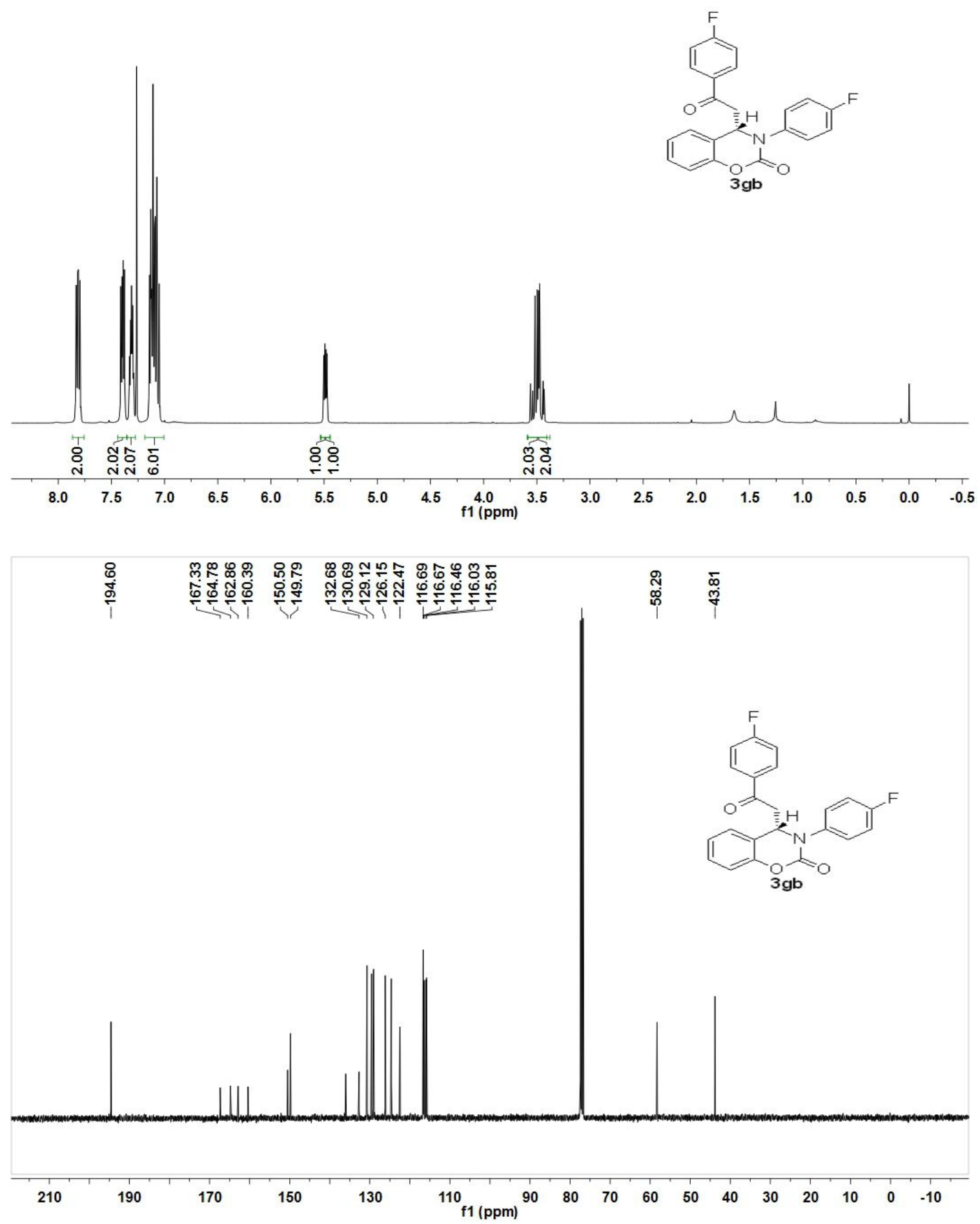


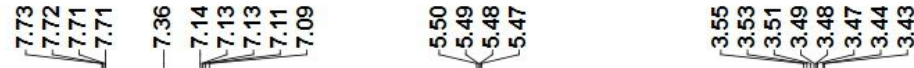
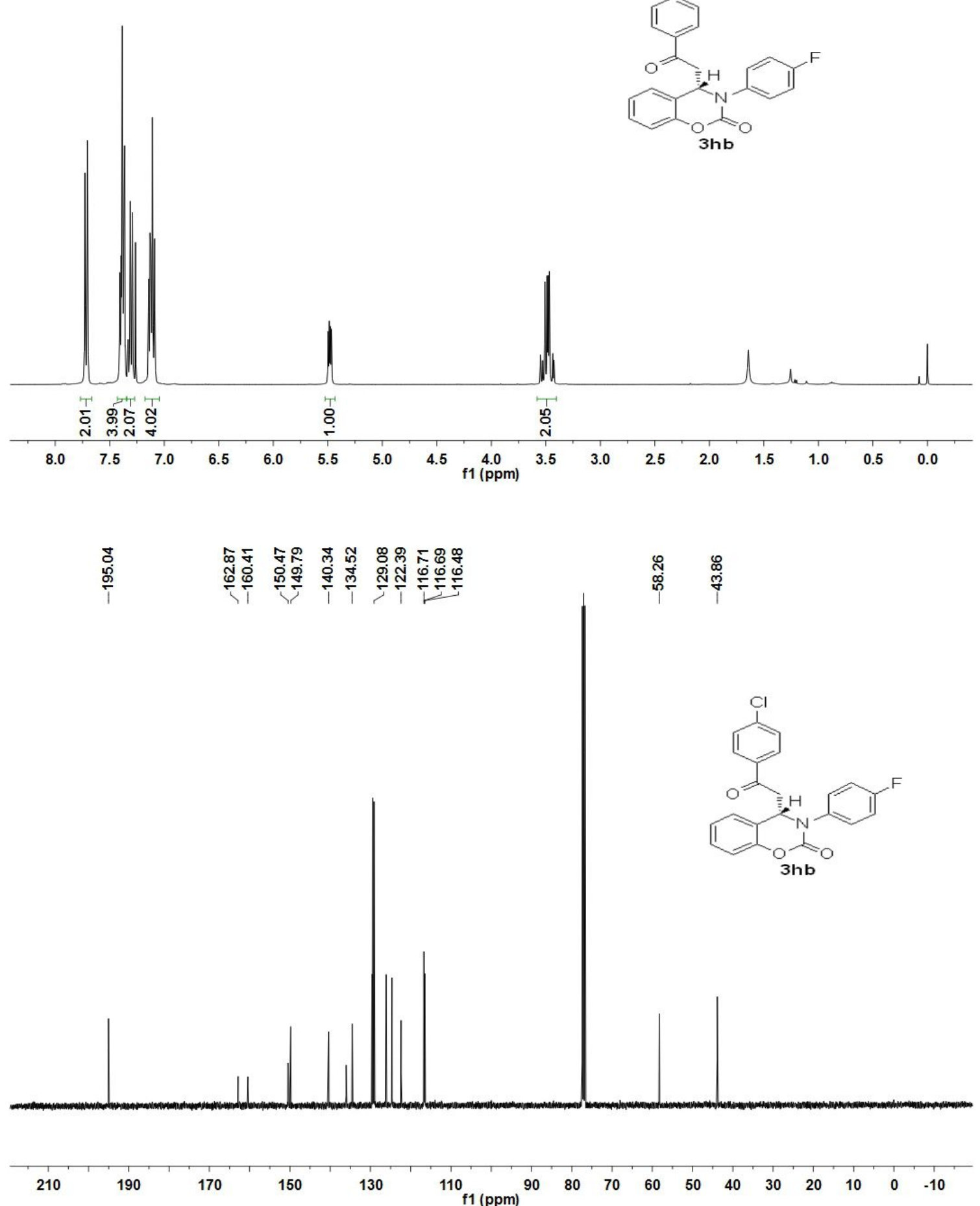

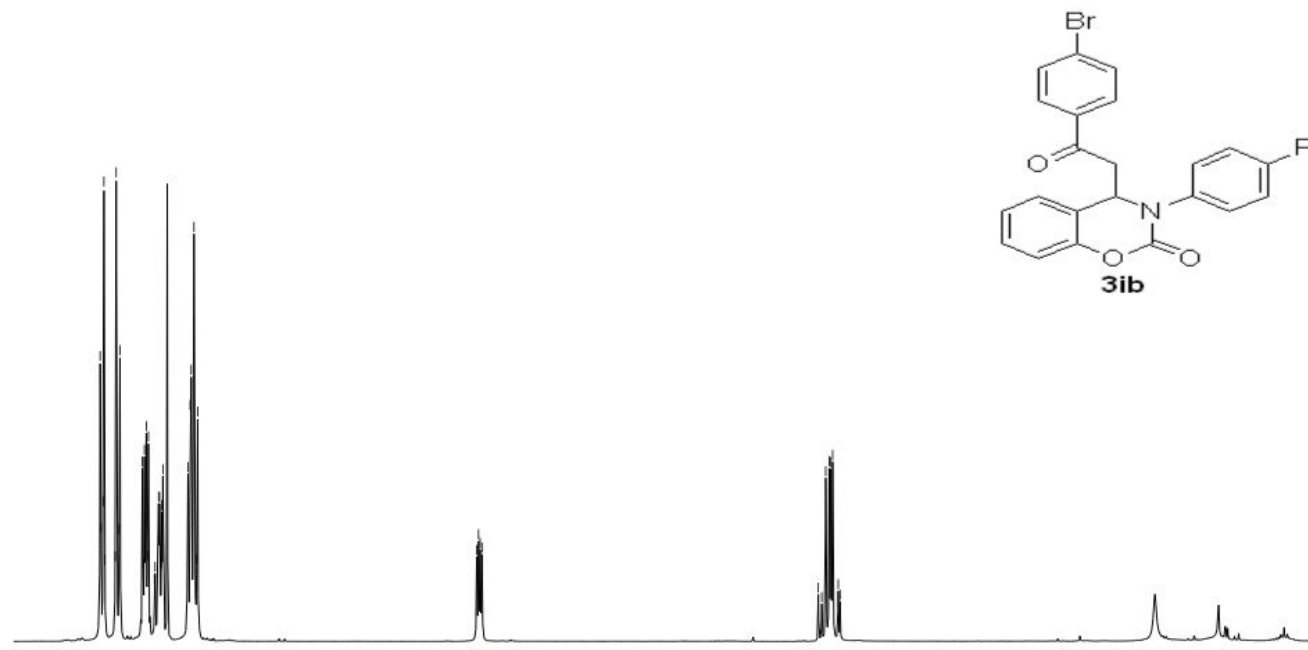

స్ำ

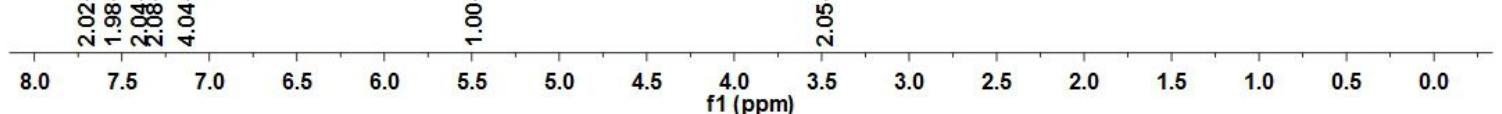

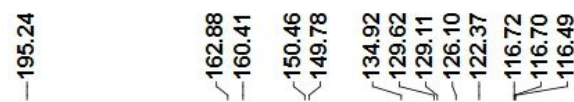

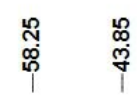
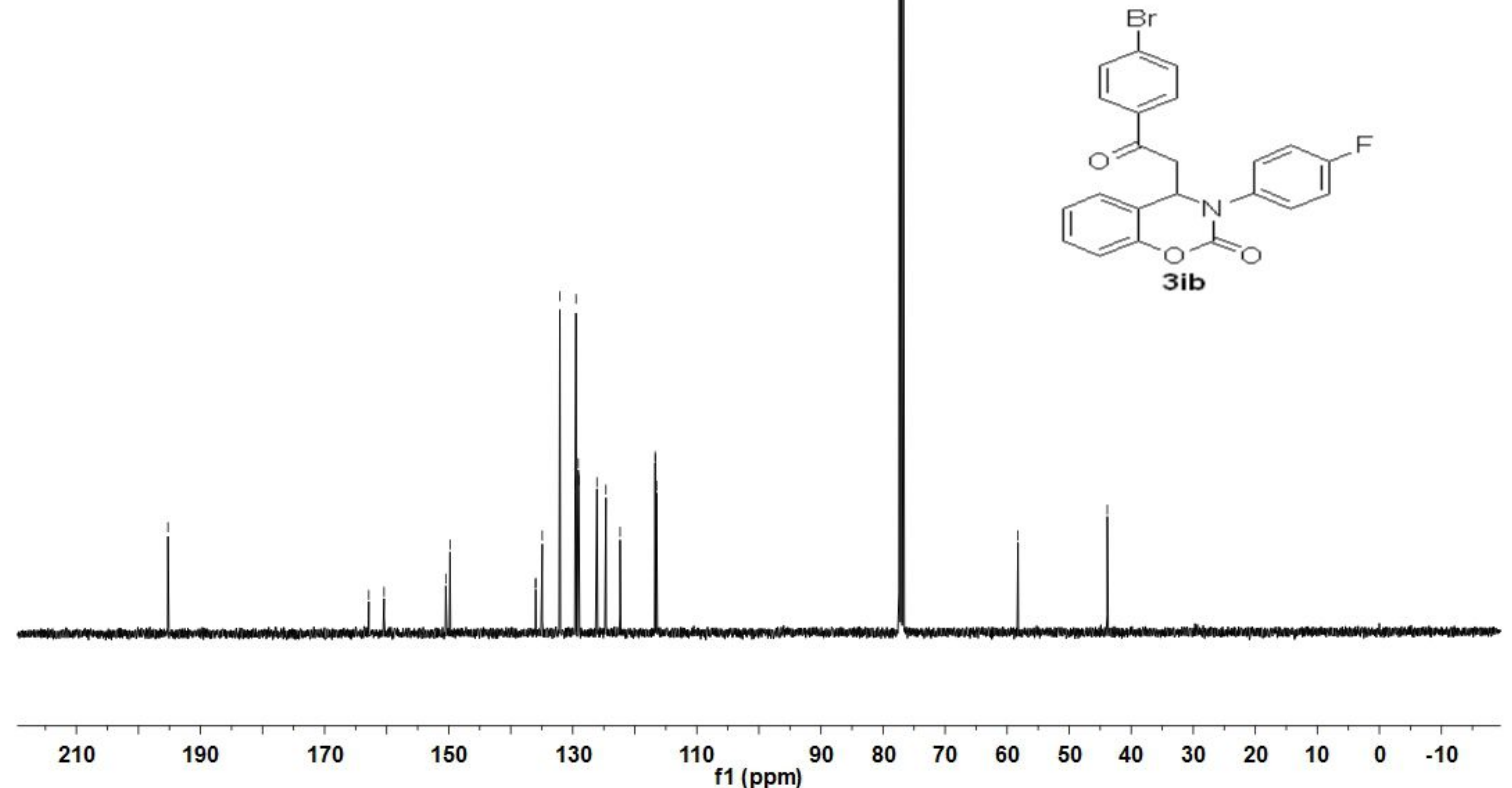


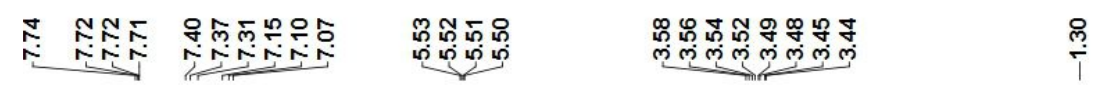

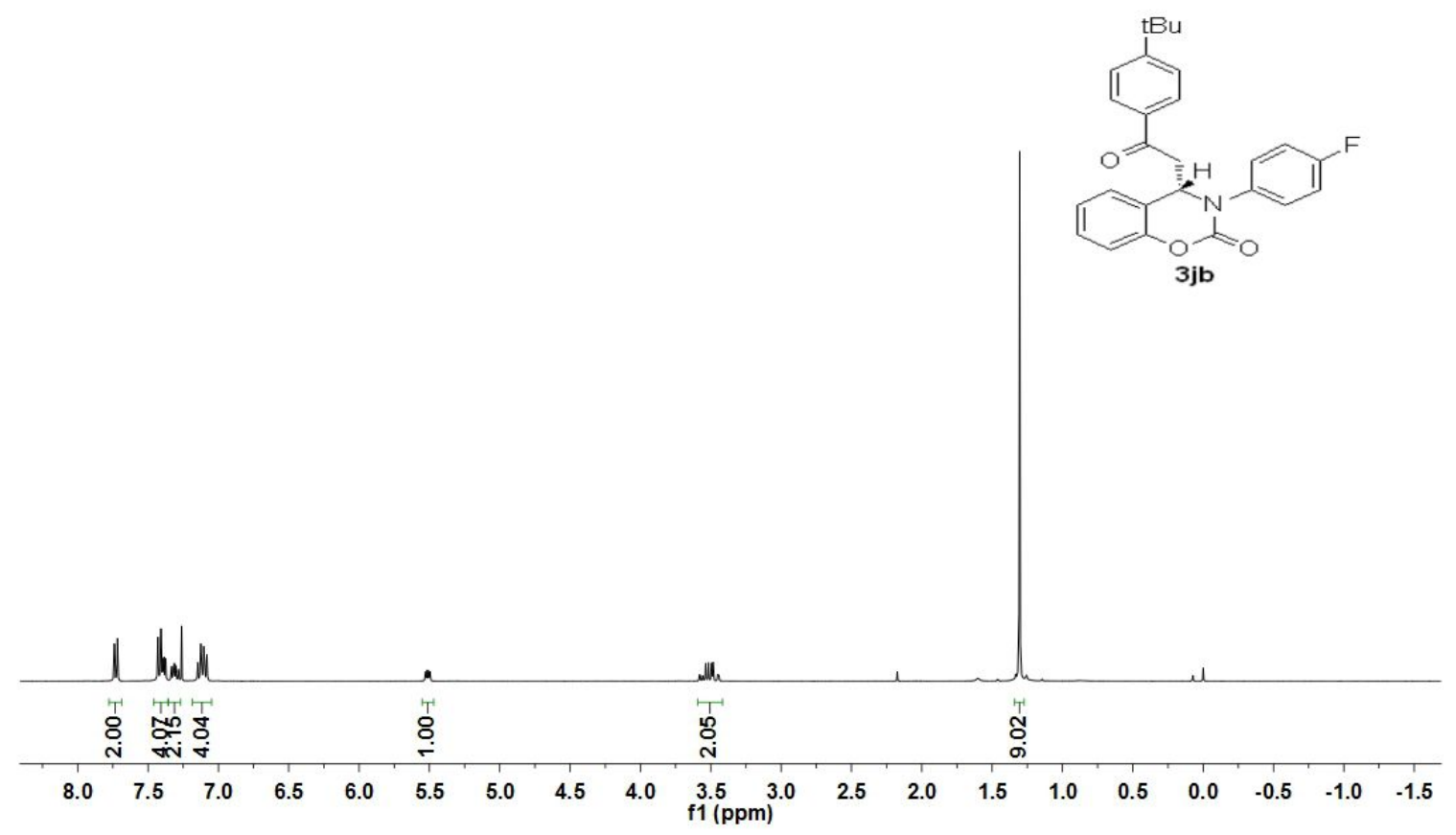

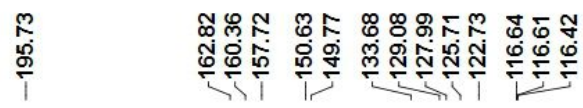

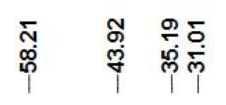

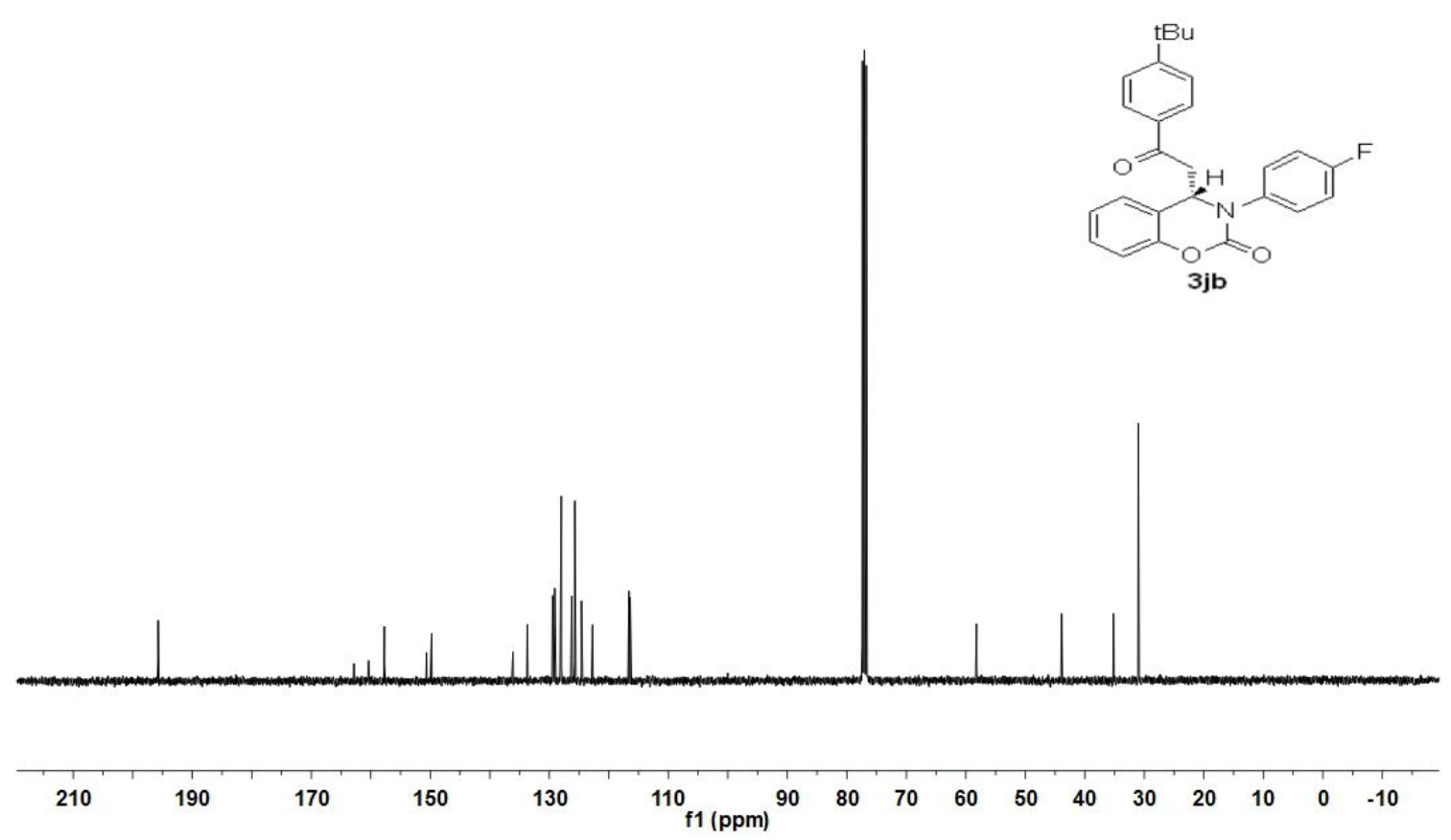




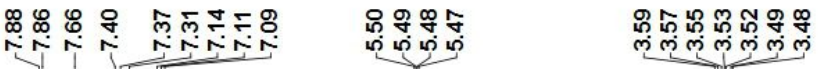
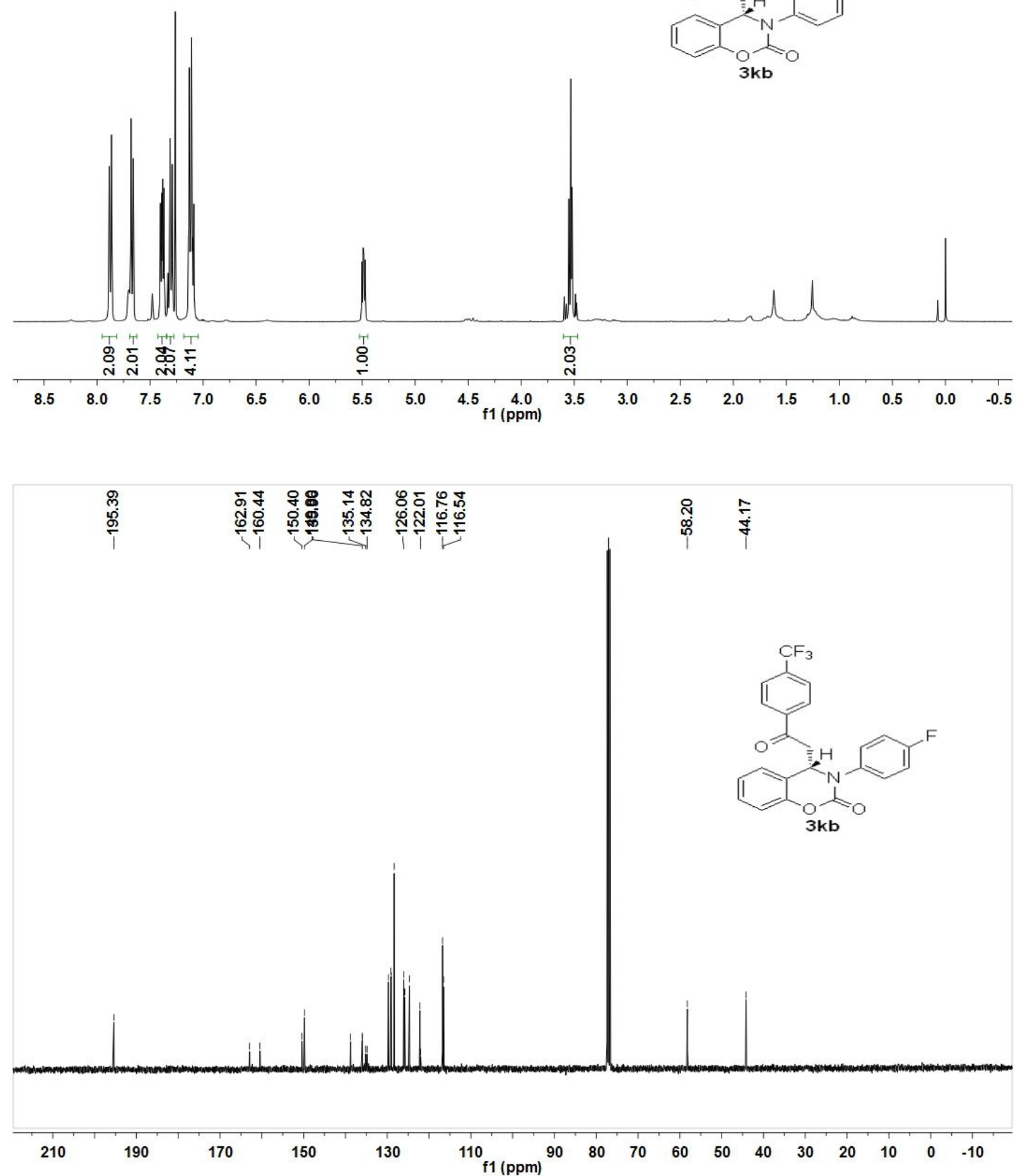


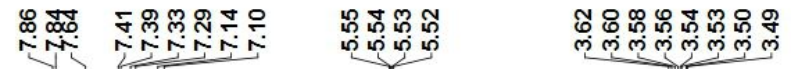
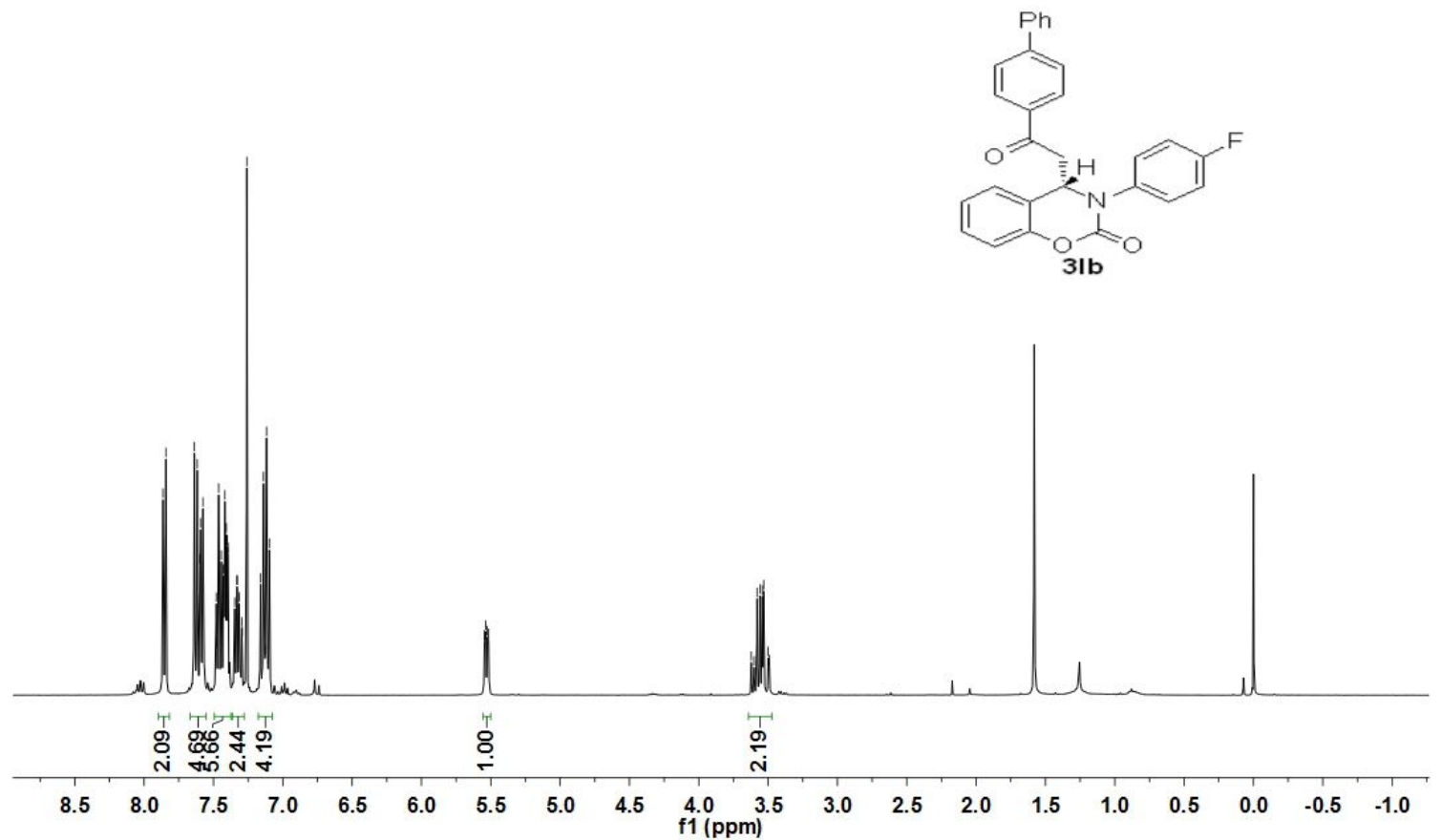

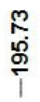

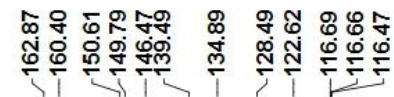

心్
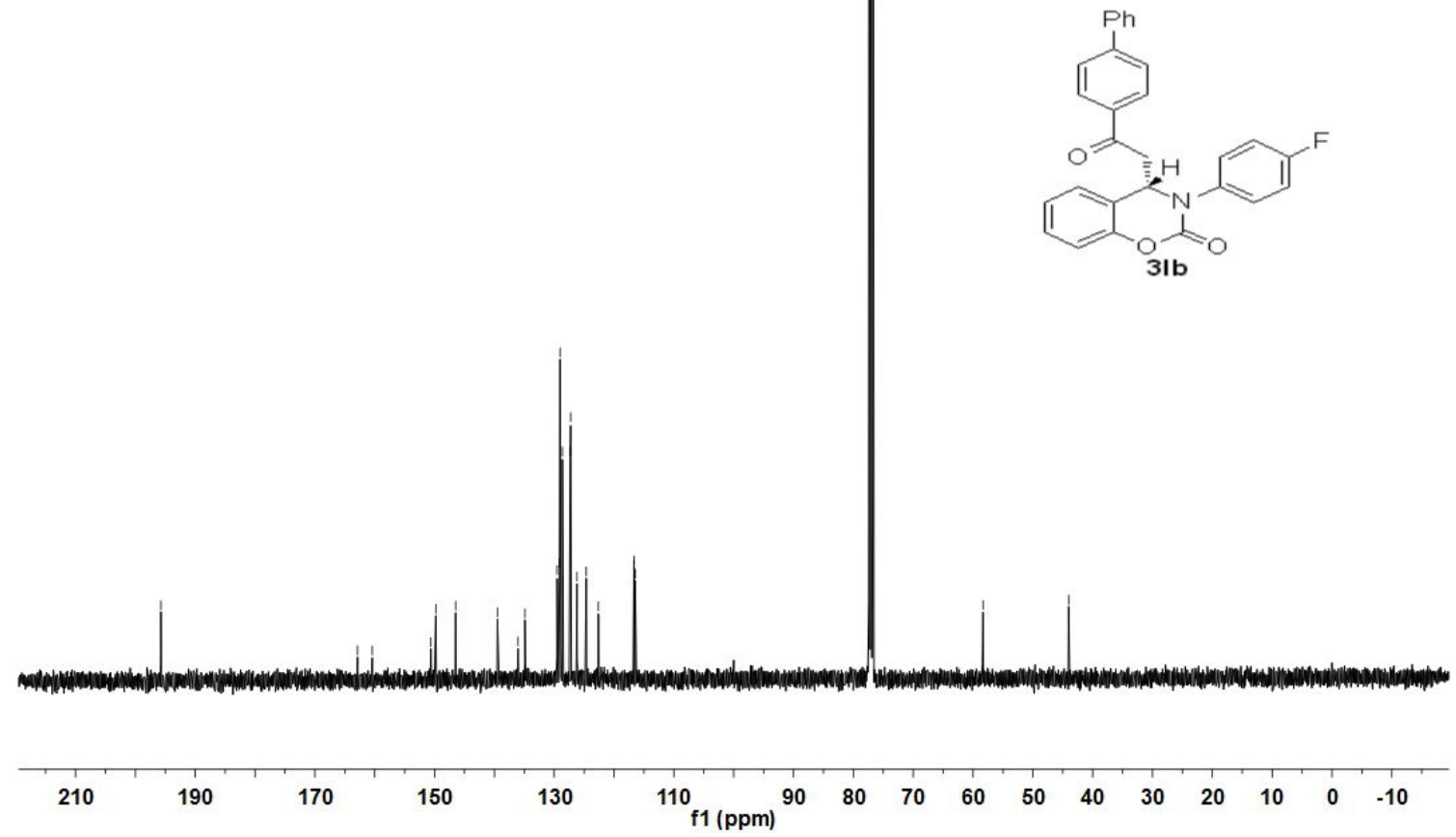

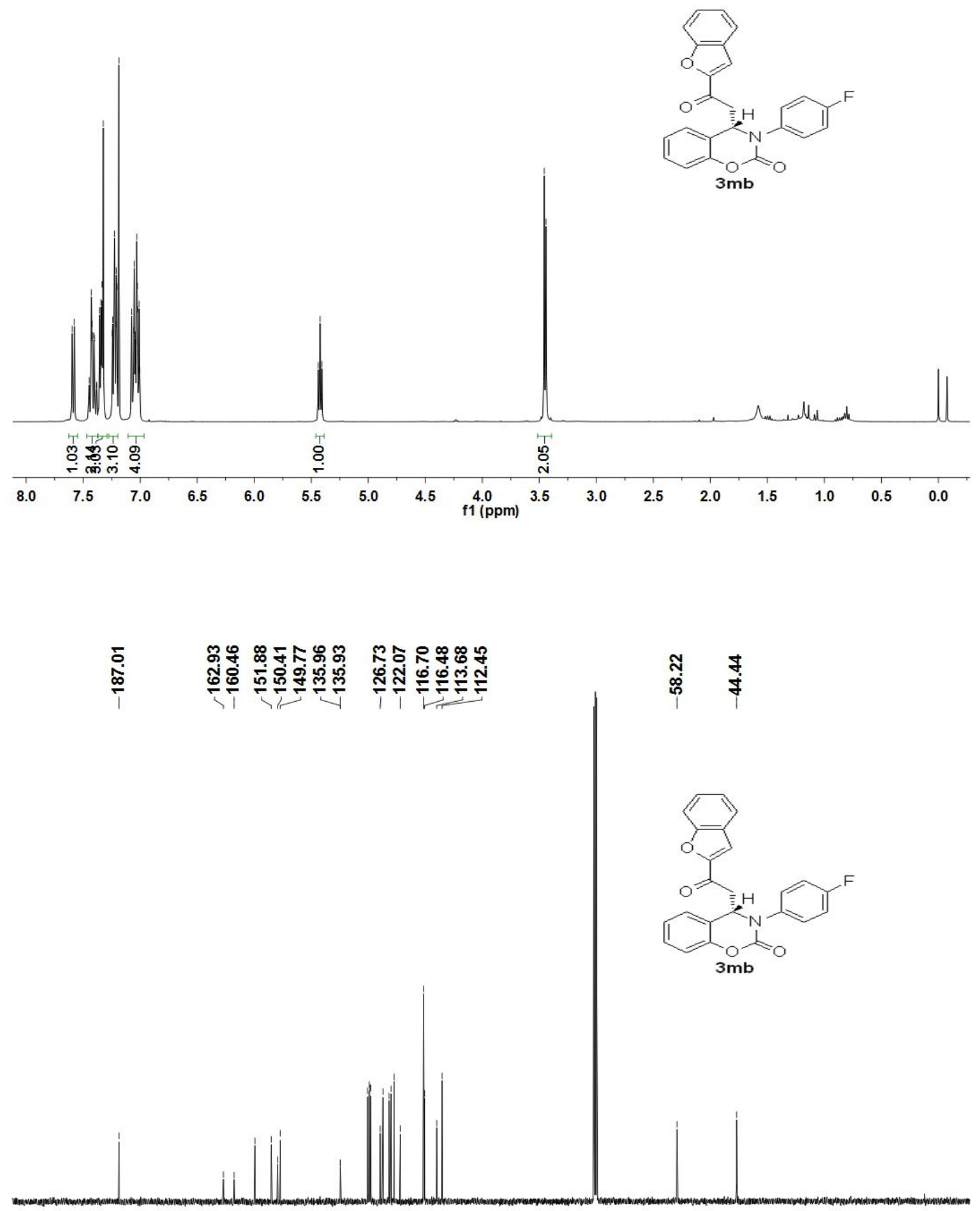

$\begin{array}{lllllllllllllllllllllll}210 & 200 & 190 & 180 & 170 & 160 & 150 & 140 & 130 & 120 & 110 & 100 & 90 & 80 & 70 & 60 & 50 & 40 & 30 & 20 & 10 & 0\end{array}$ 

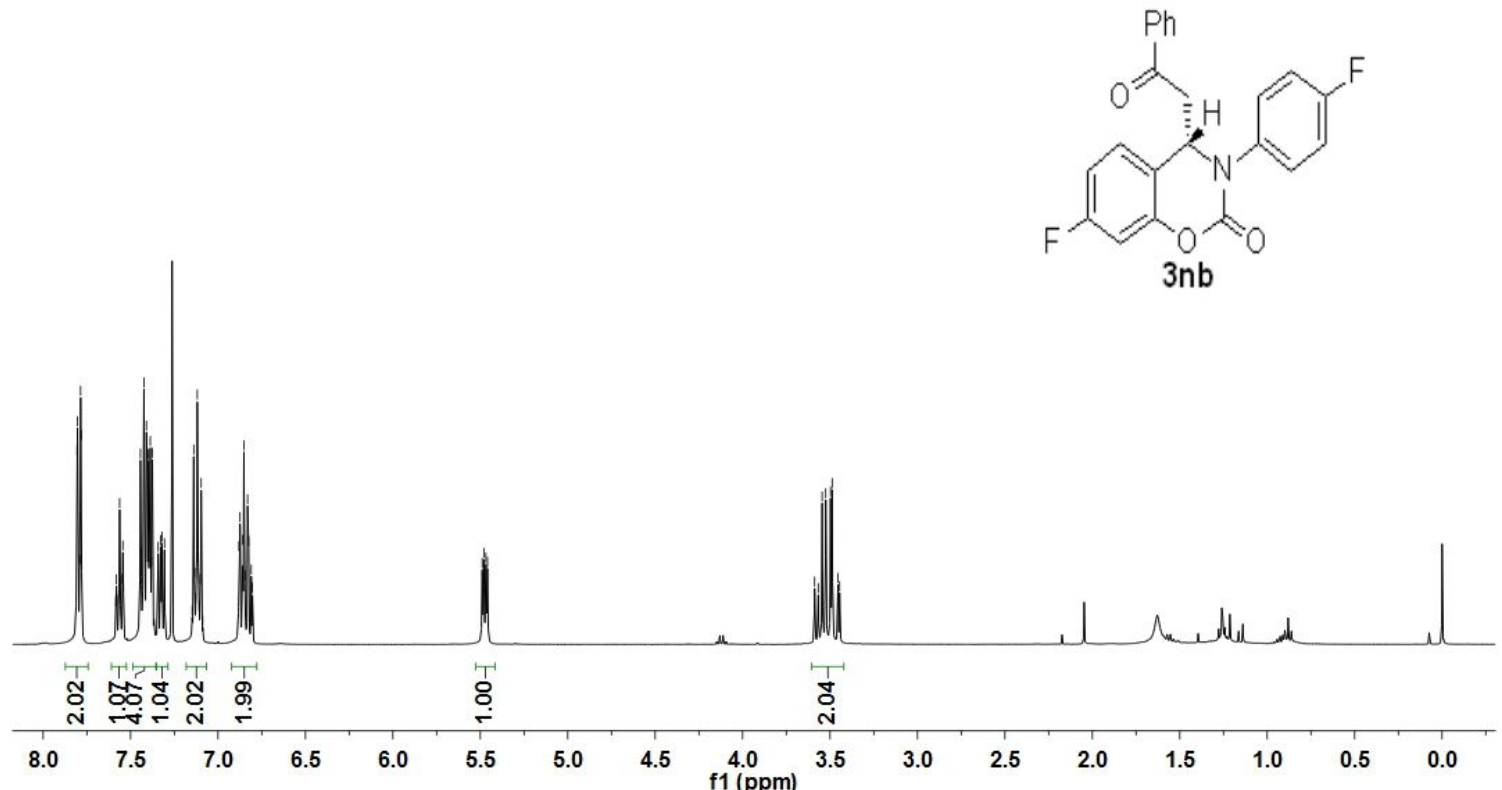

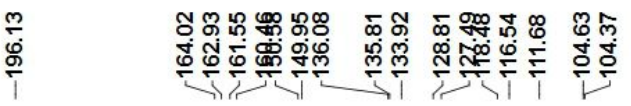

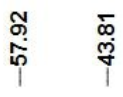
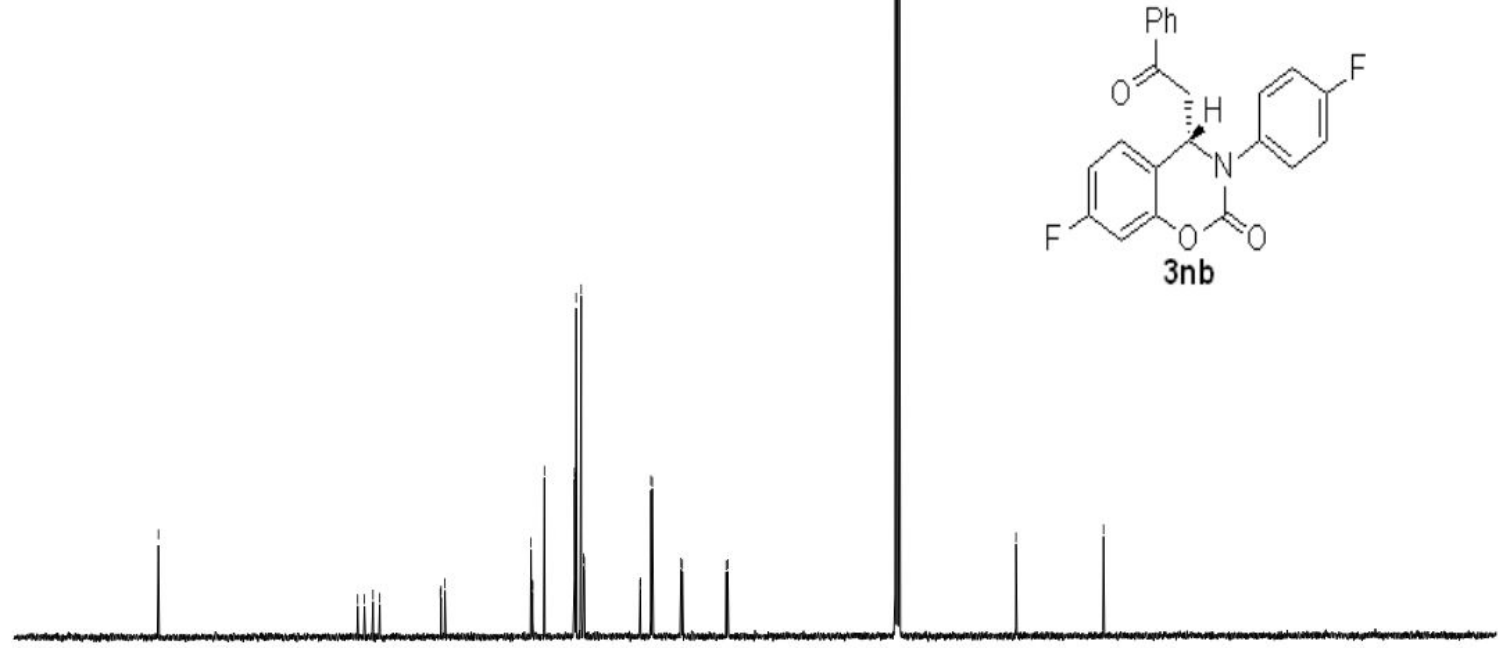

$\begin{array}{llllllllllllllllll}210 & 190 & 170 & 150 & 130 & 110 & \mathrm{f} 1(\mathrm{ppm}) & 90 & 80 & 70 & 60 & 50 & 40 & 30 & 20 & 10 & 0 & -10\end{array}$ 


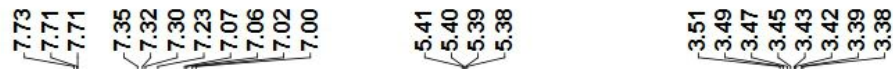
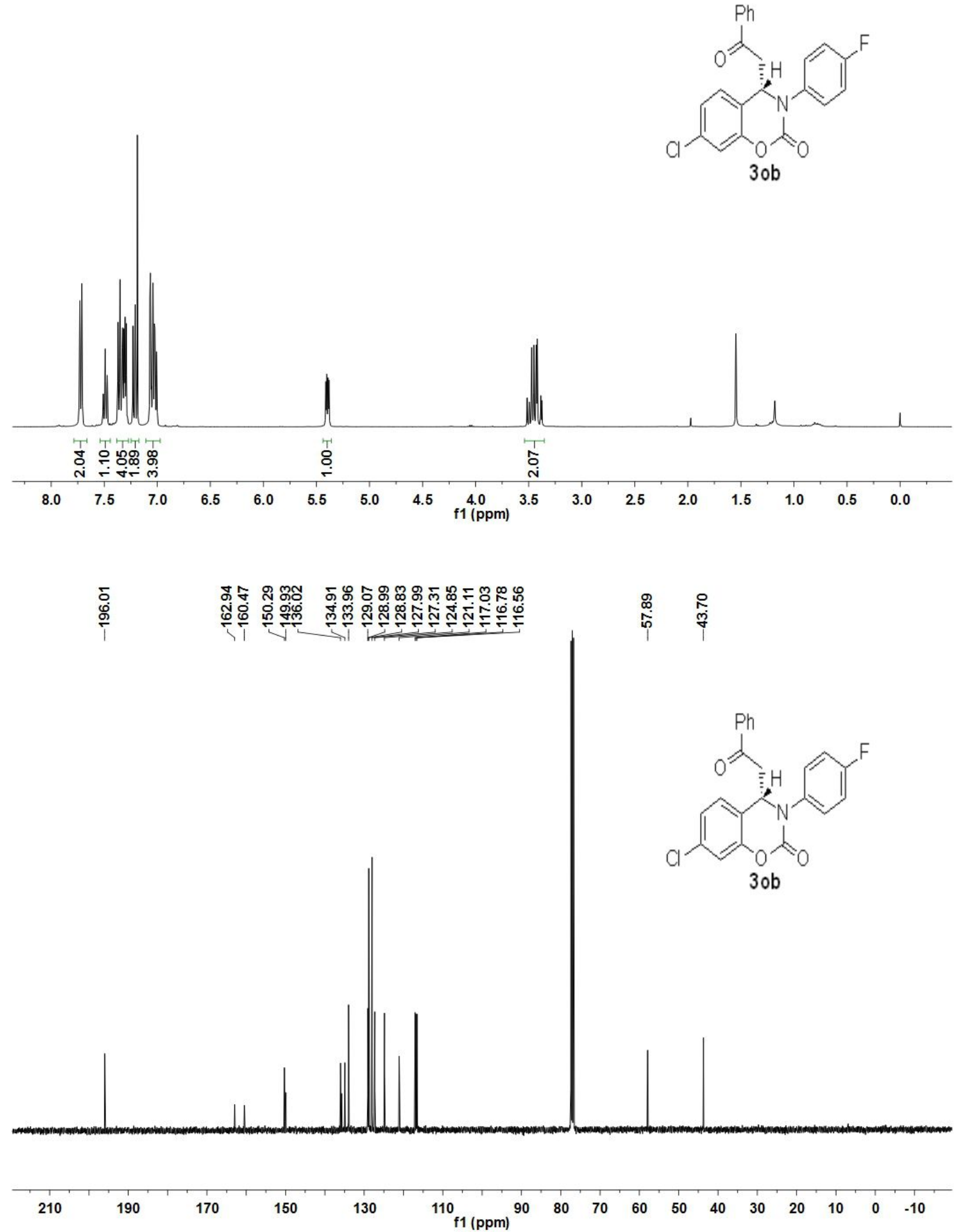

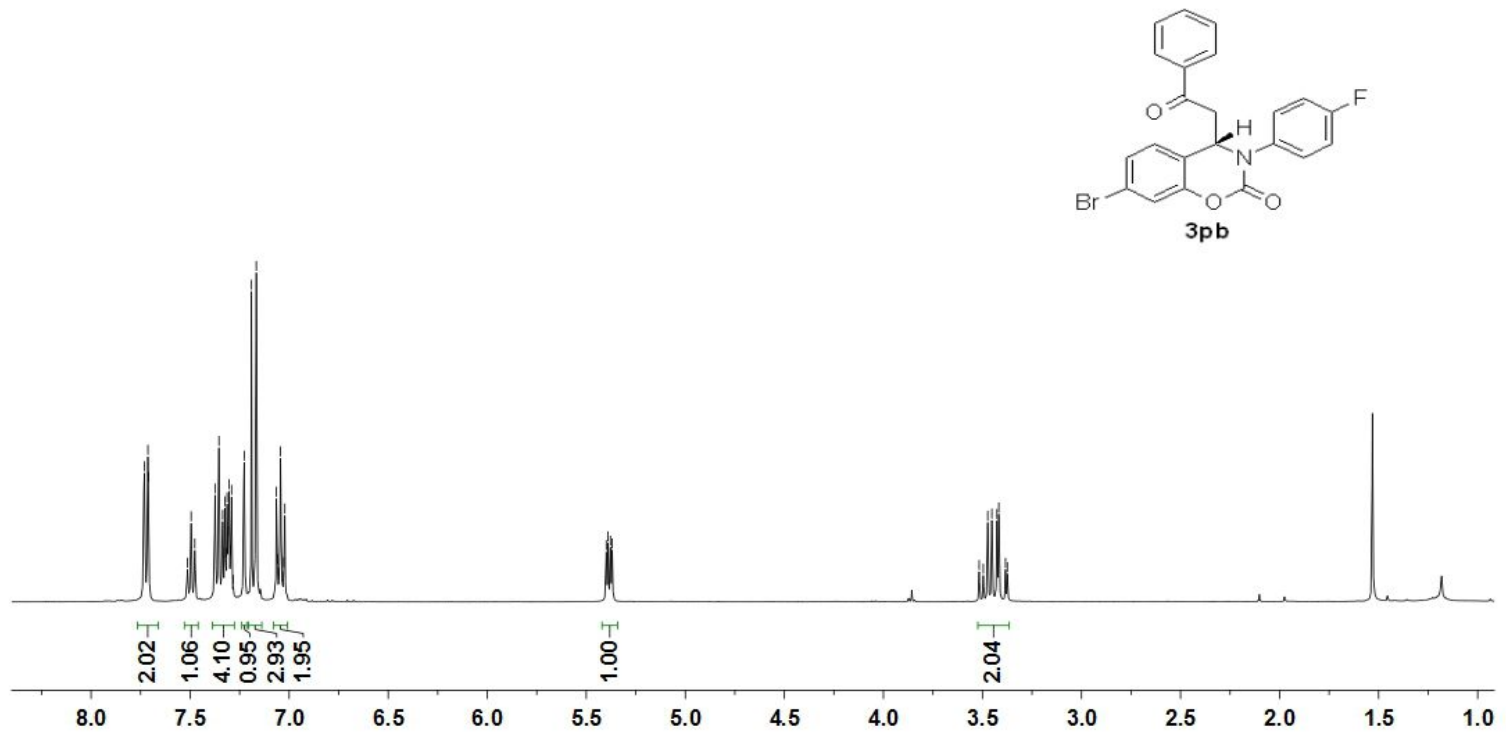

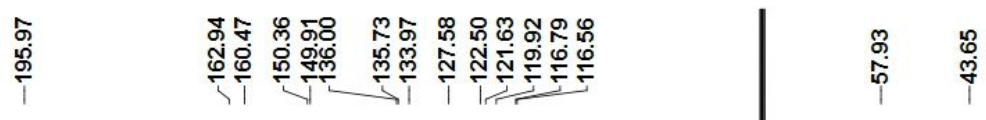
$210 \quad 190$
$170 \quad 150$
130 ${ }_{\mathrm{f} 1(\mathrm{ppm})}^{110} 90$
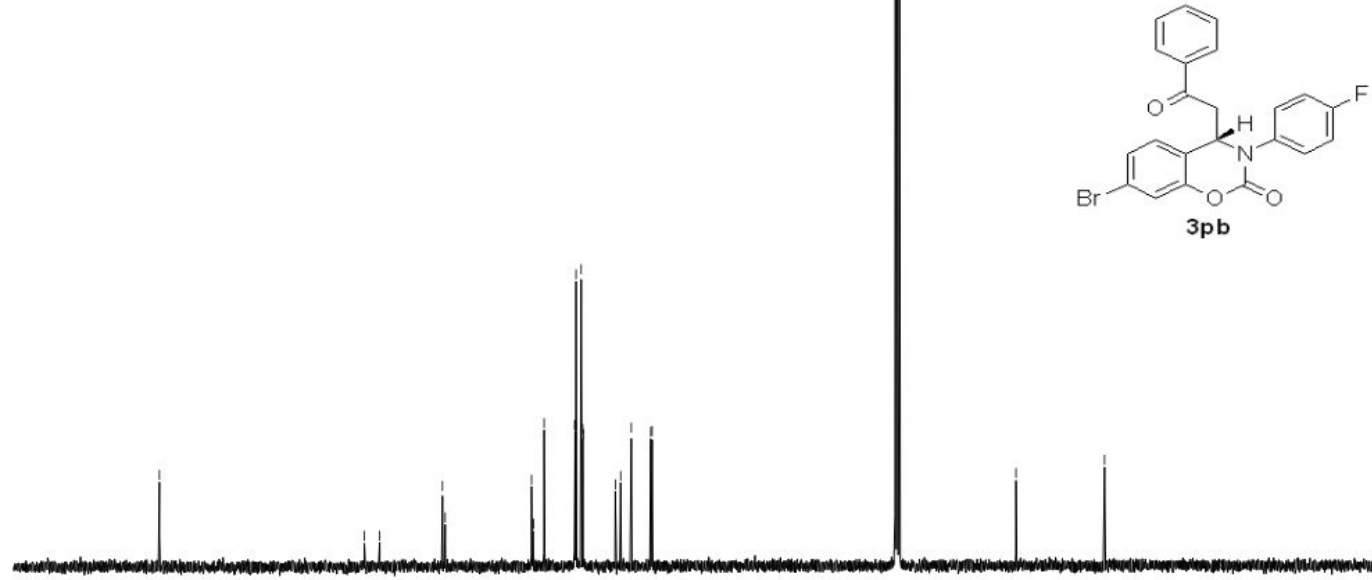

\section{$\stackrel{\leftrightarrow}{\dagger}$}




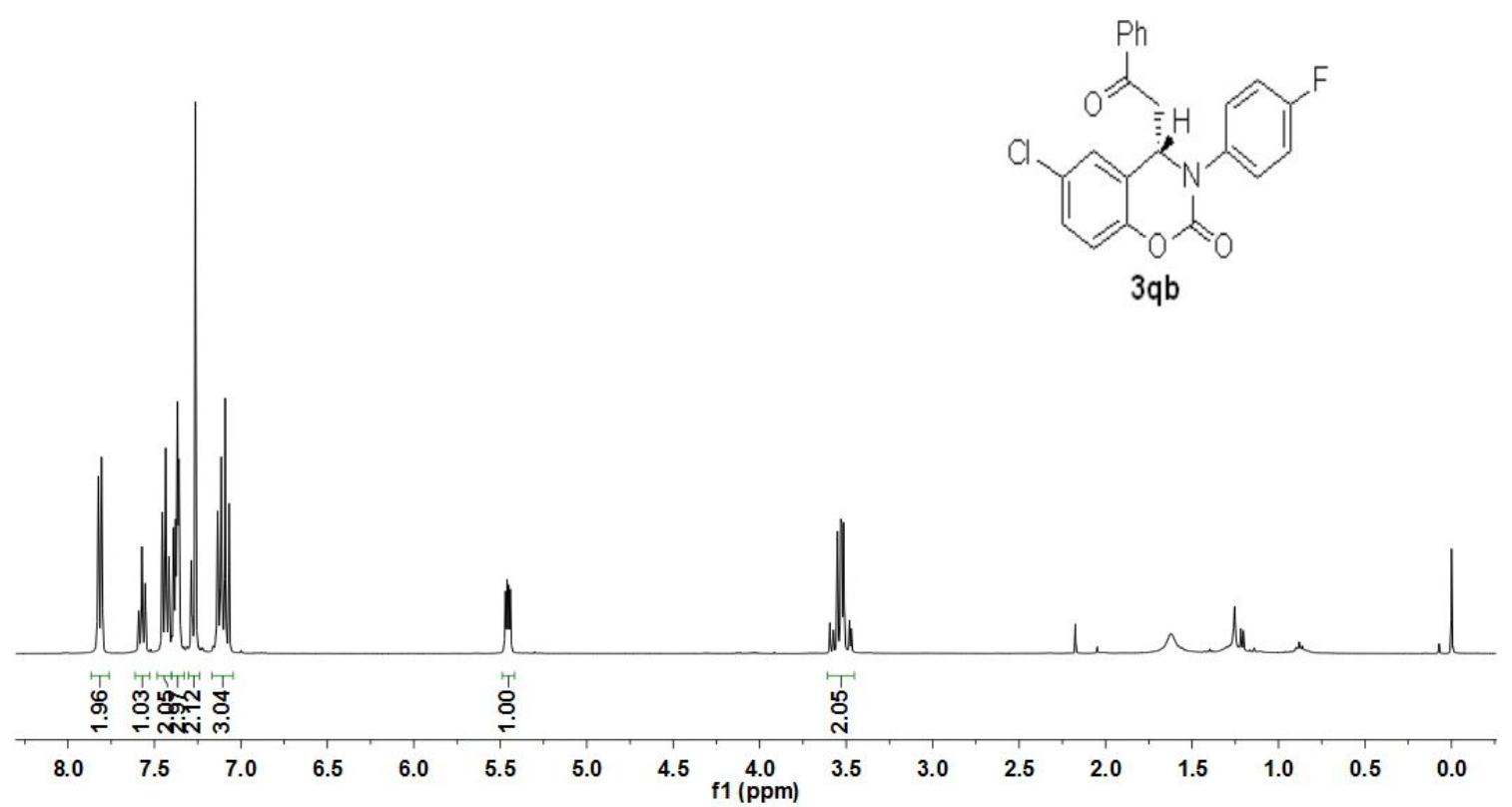

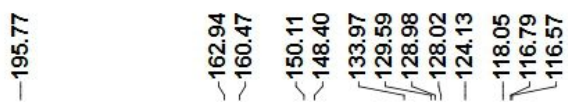

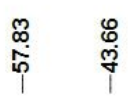

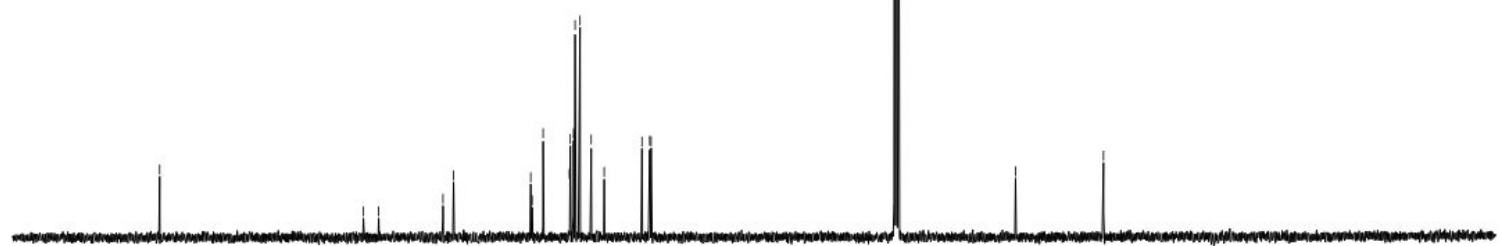

$\begin{array}{llllllllllllllllllllll}210 & 190 & 170 & 150 & 130 & 110 & 90 & 80 & 70 & 60 & 50 & 40 & 30 & 20 & 10 & 0 & -10\end{array}$ 

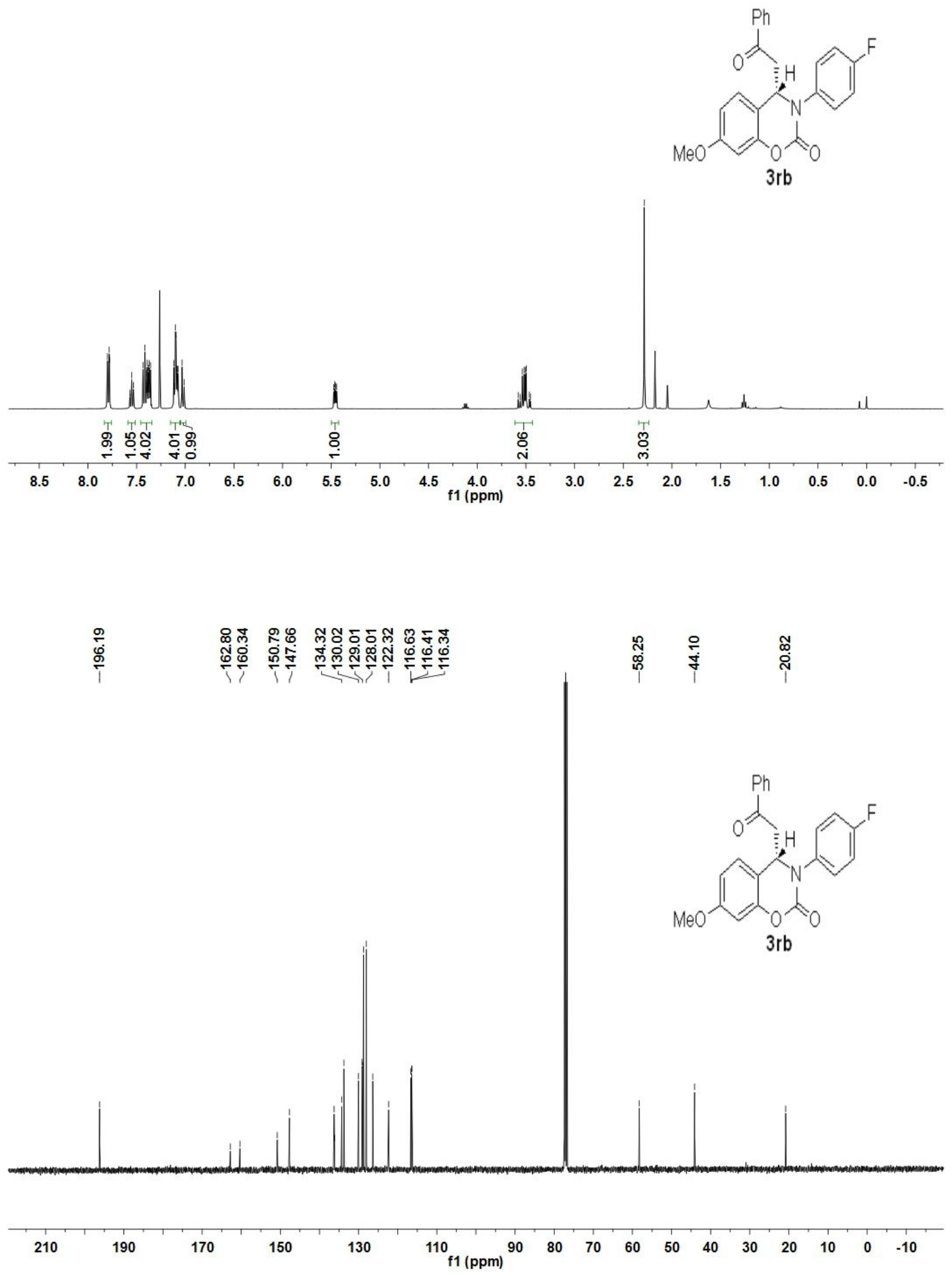


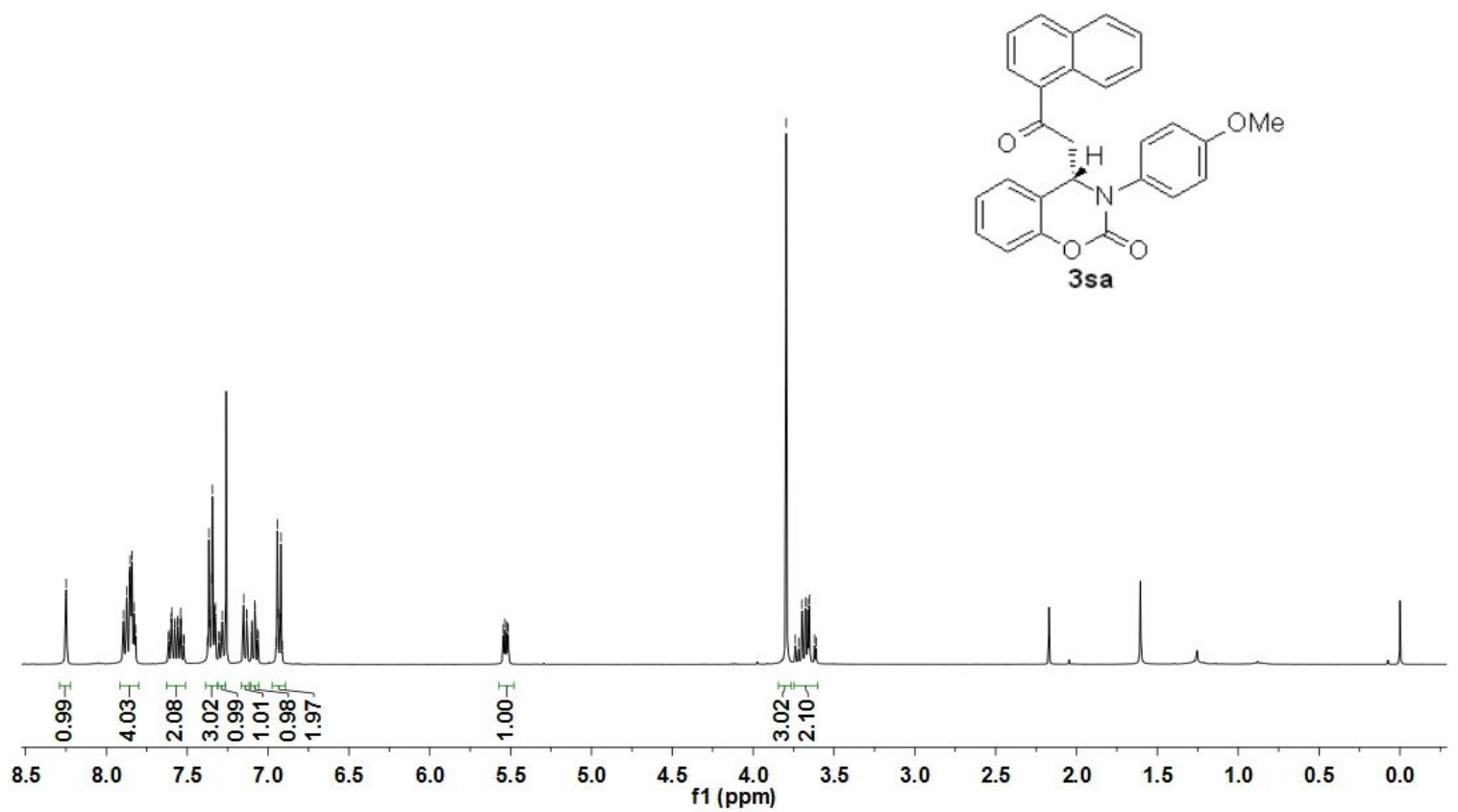

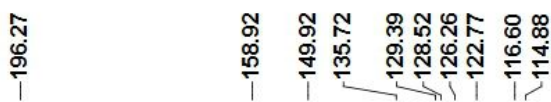

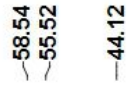

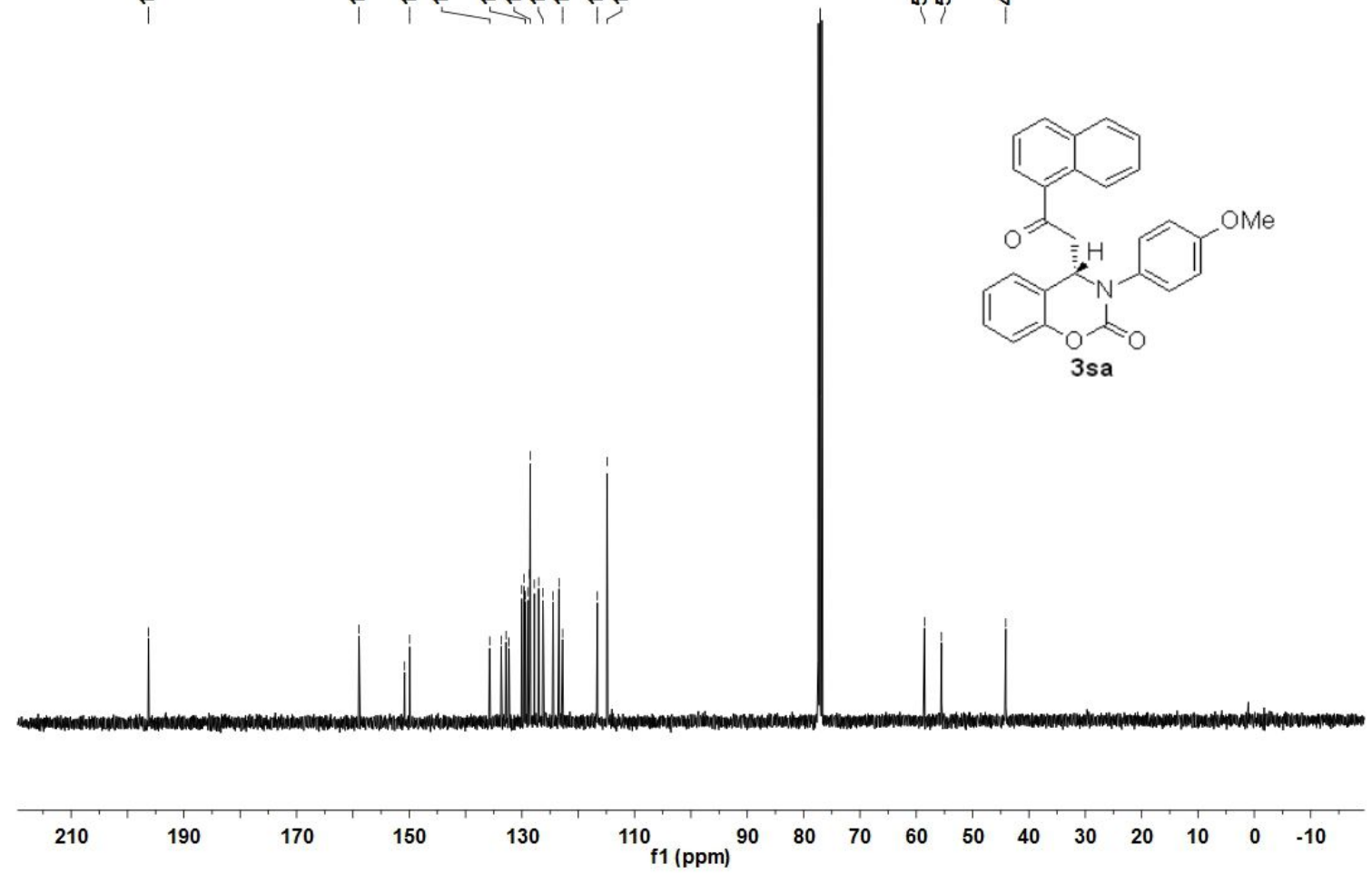




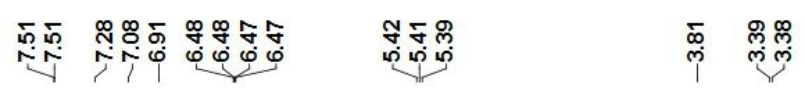
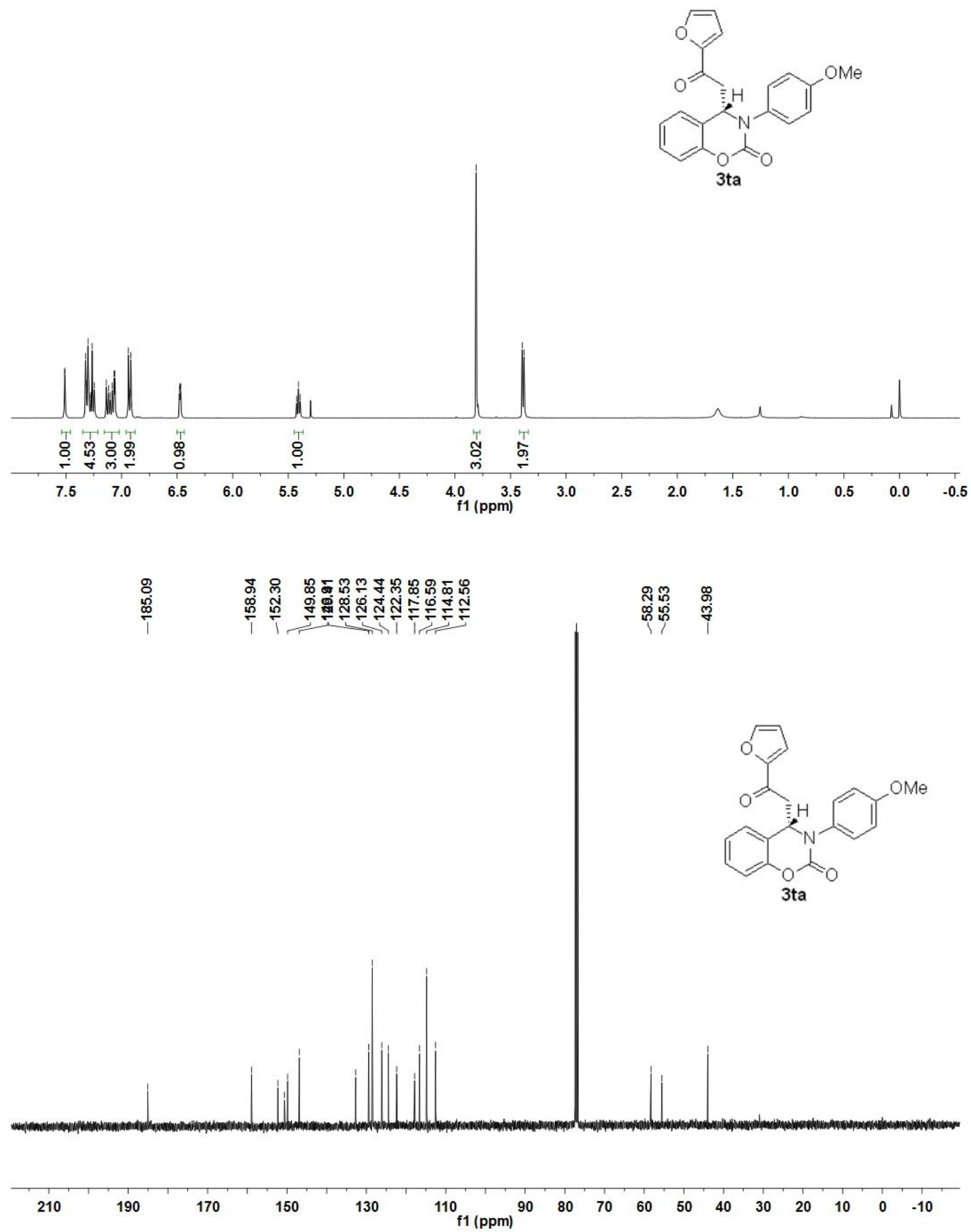

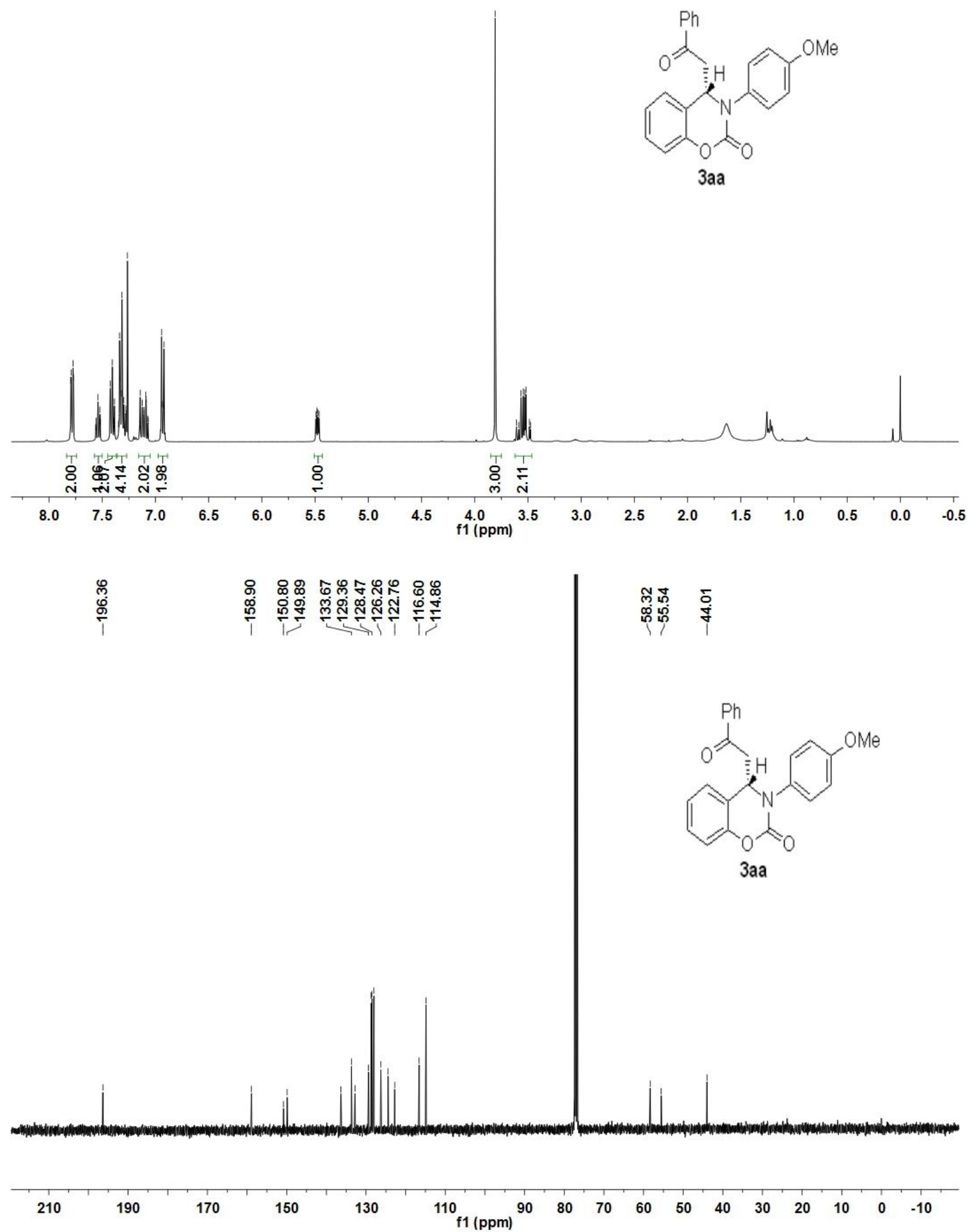


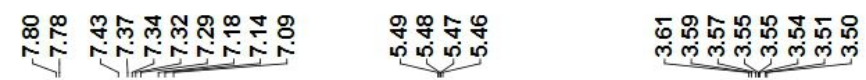
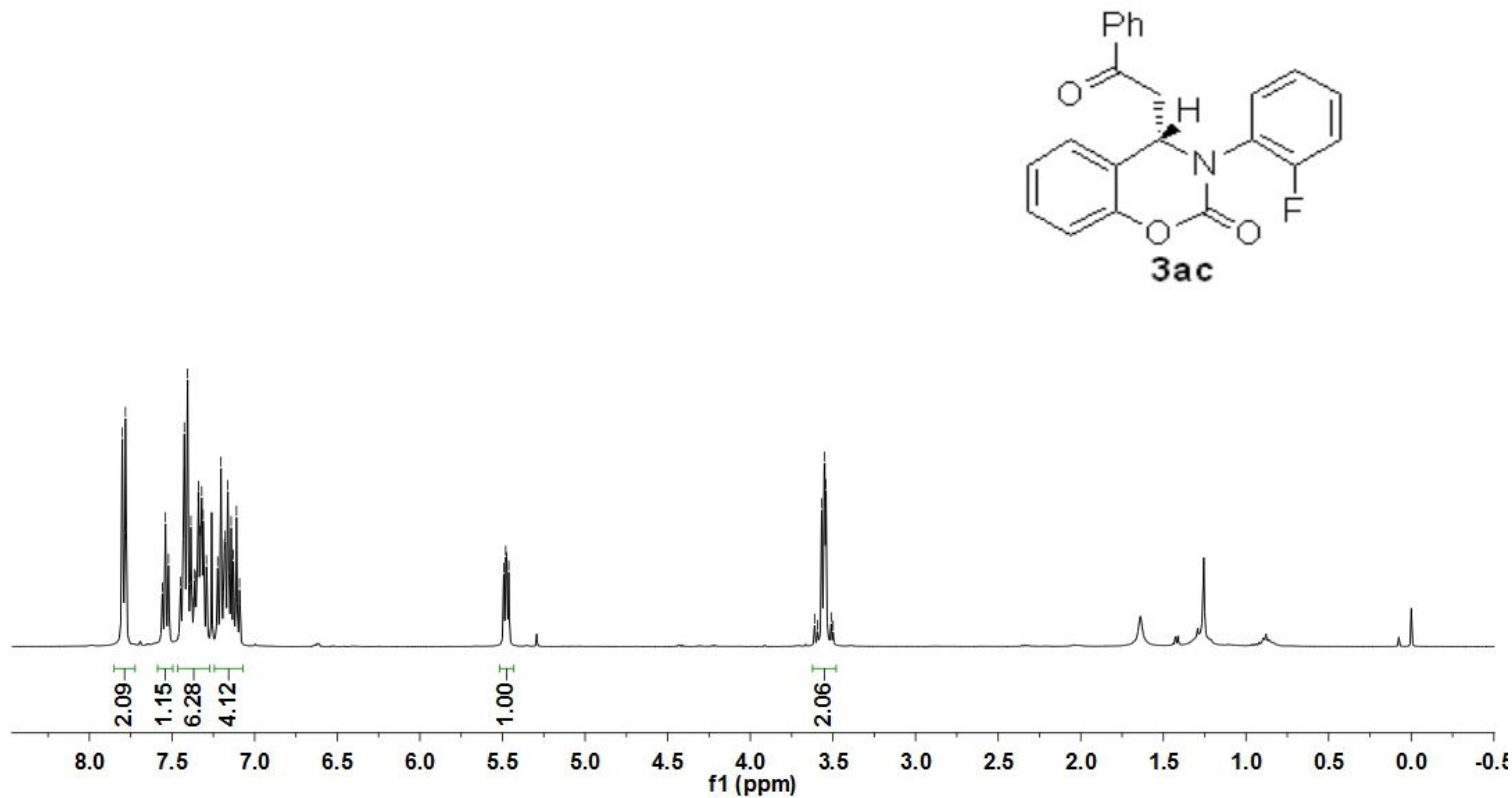

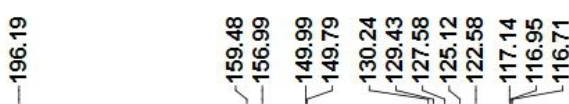

$\stackrel{\substack{\infty \\ i}}{\stackrel{j}{j}}$

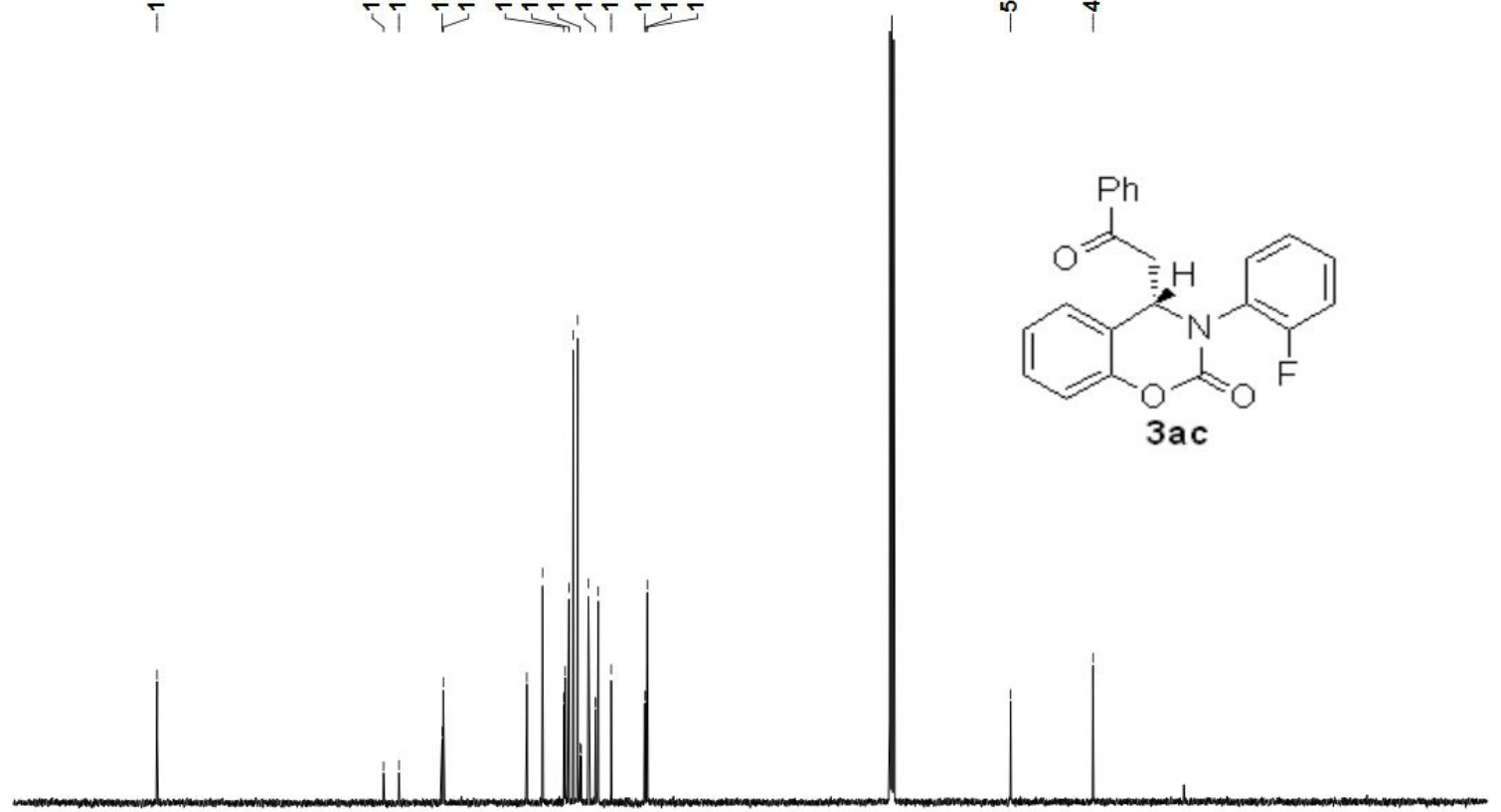

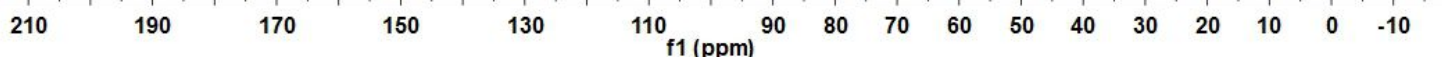




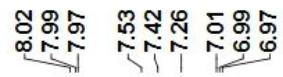
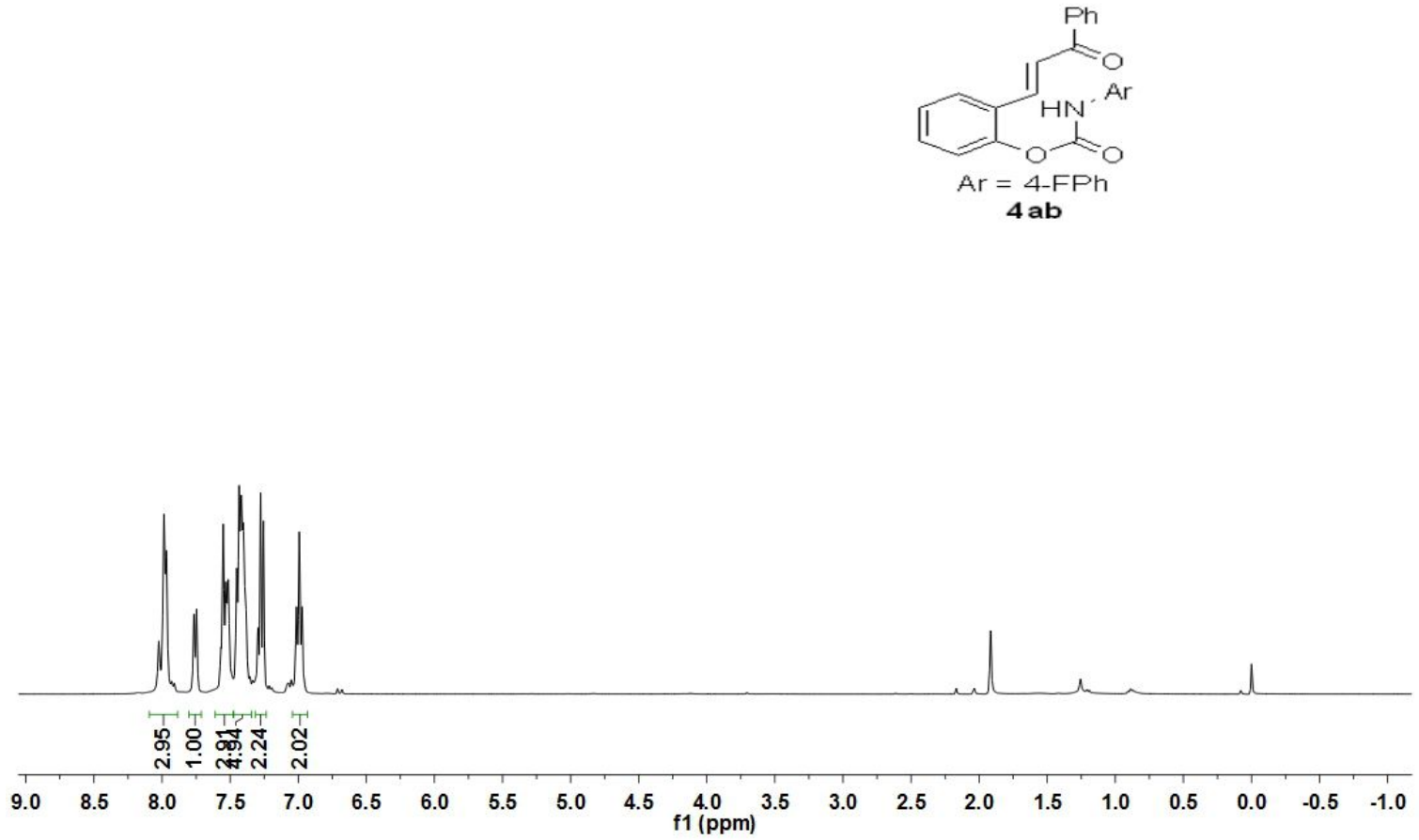

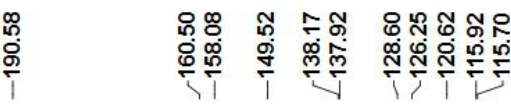

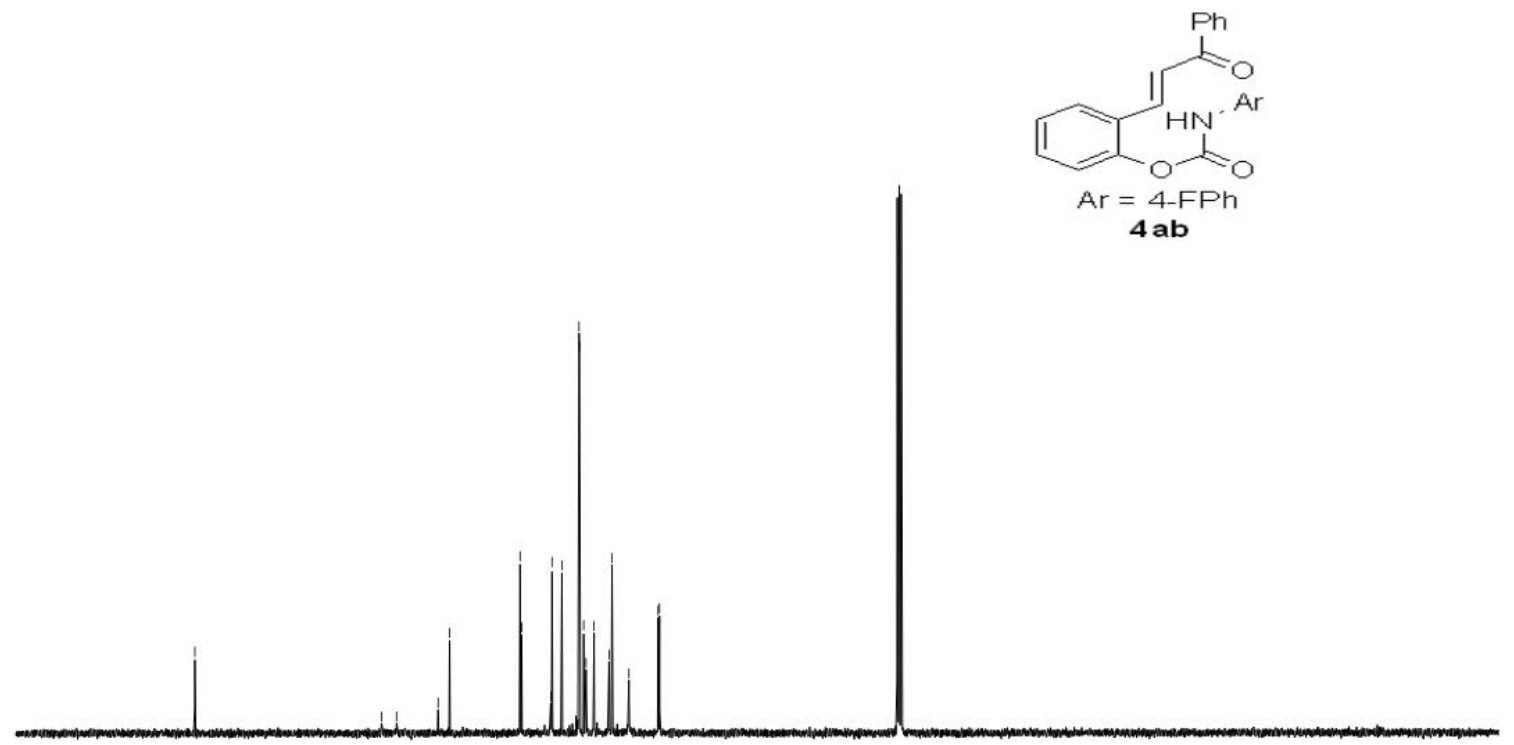

$\begin{array}{lllllllllllllllll}210 & 190 & 170 & 150 & 130 & 110_{\mathrm{f} 1(\mathrm{ppm})} & 90 & 80 & 70 & 60 & 50 & 40 & 30 & 20 & 10 & 0 & -10\end{array}$ 

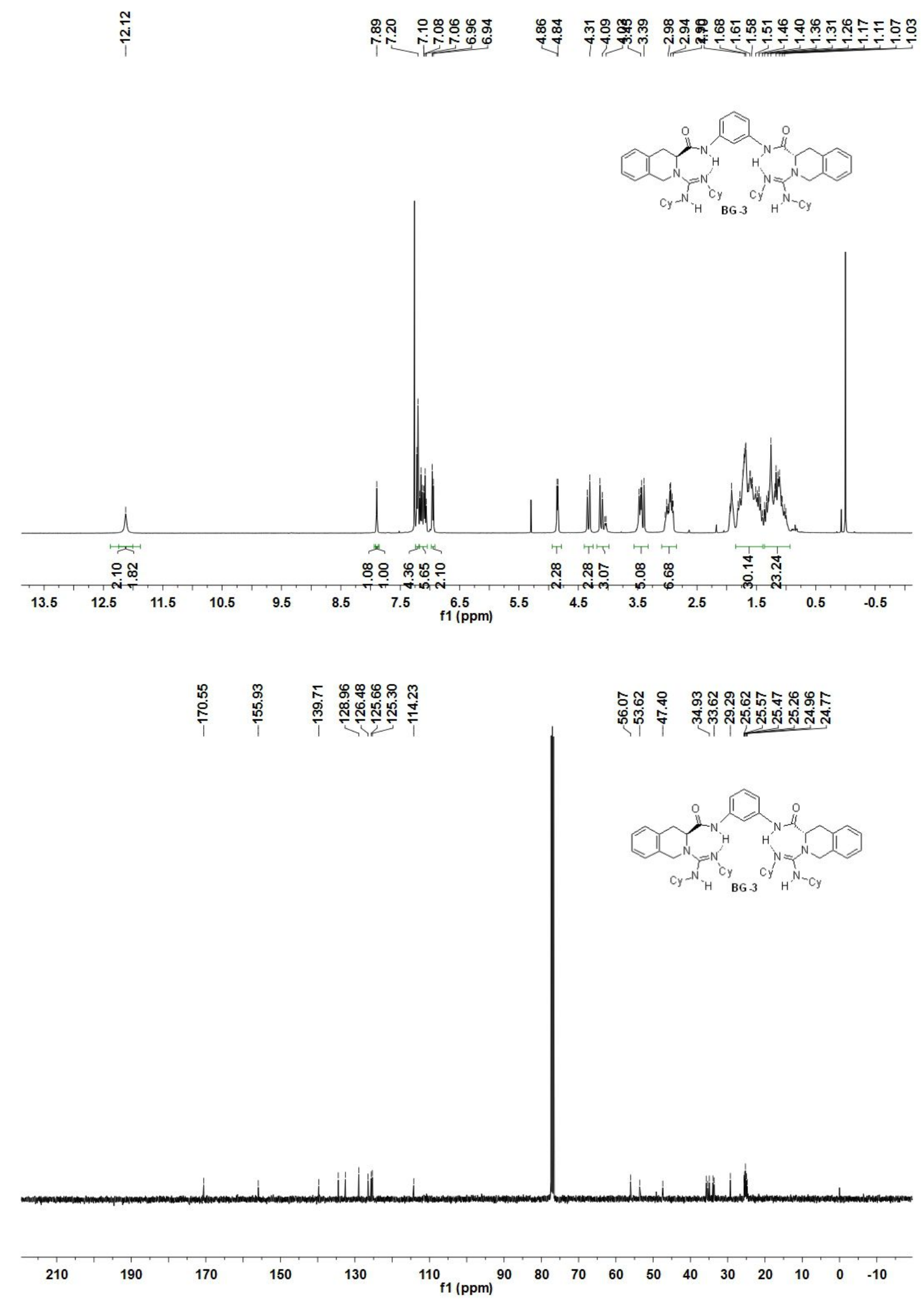

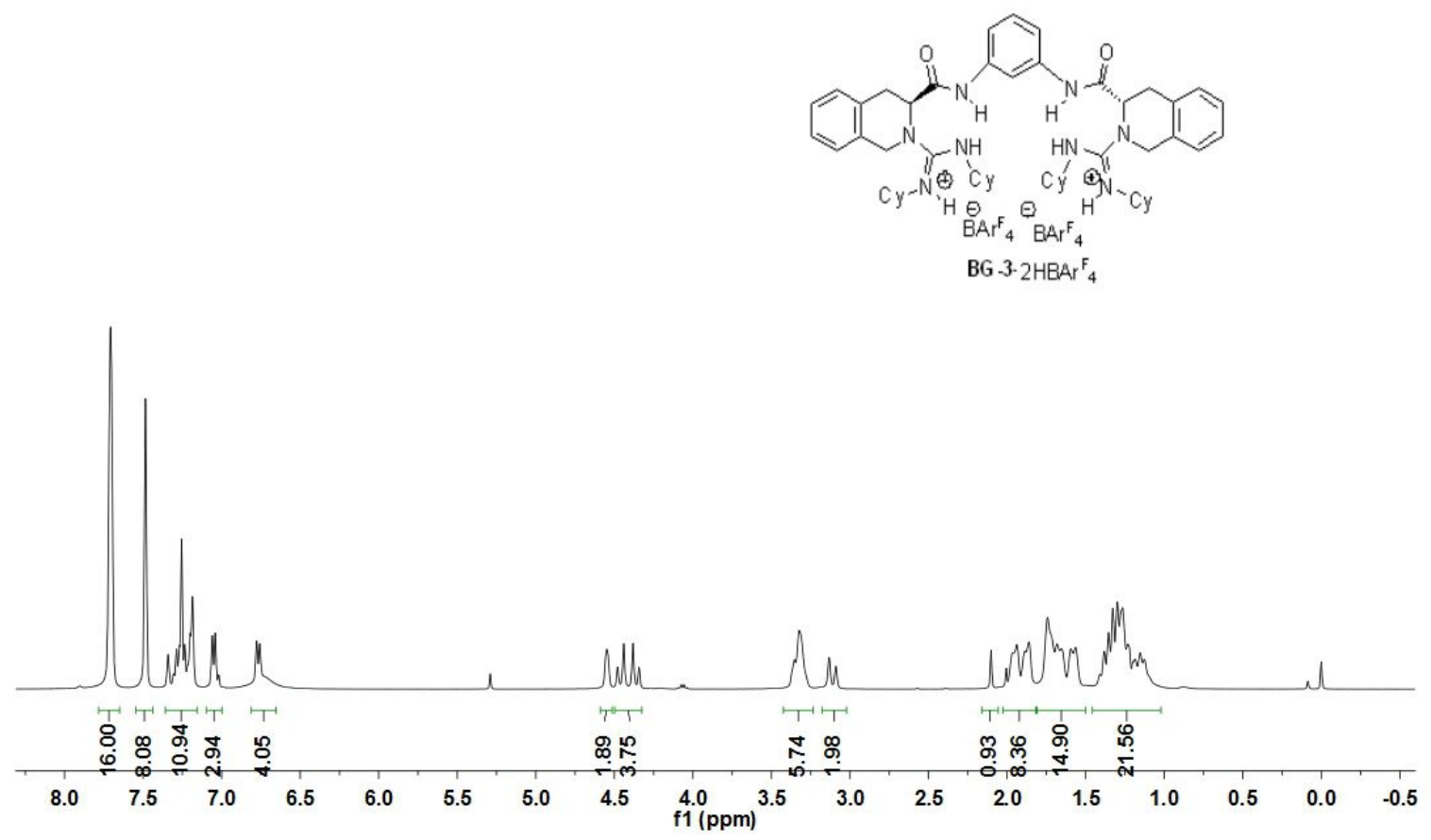

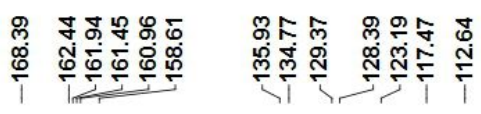

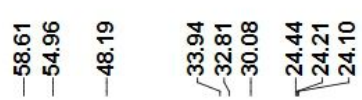
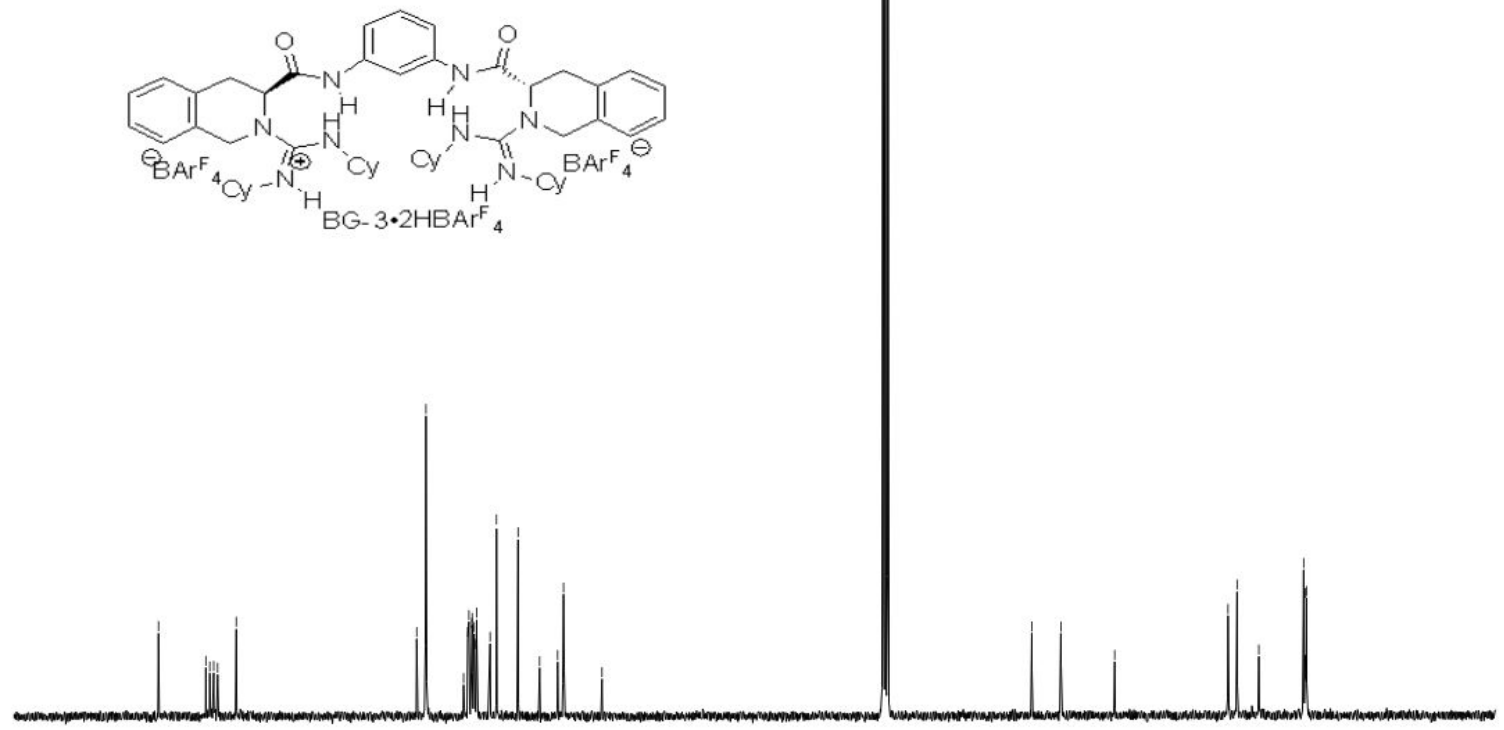

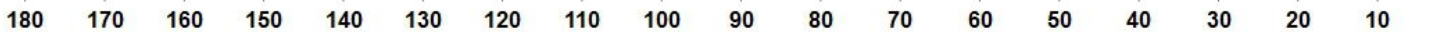


BG-3.1.3HBAF 4

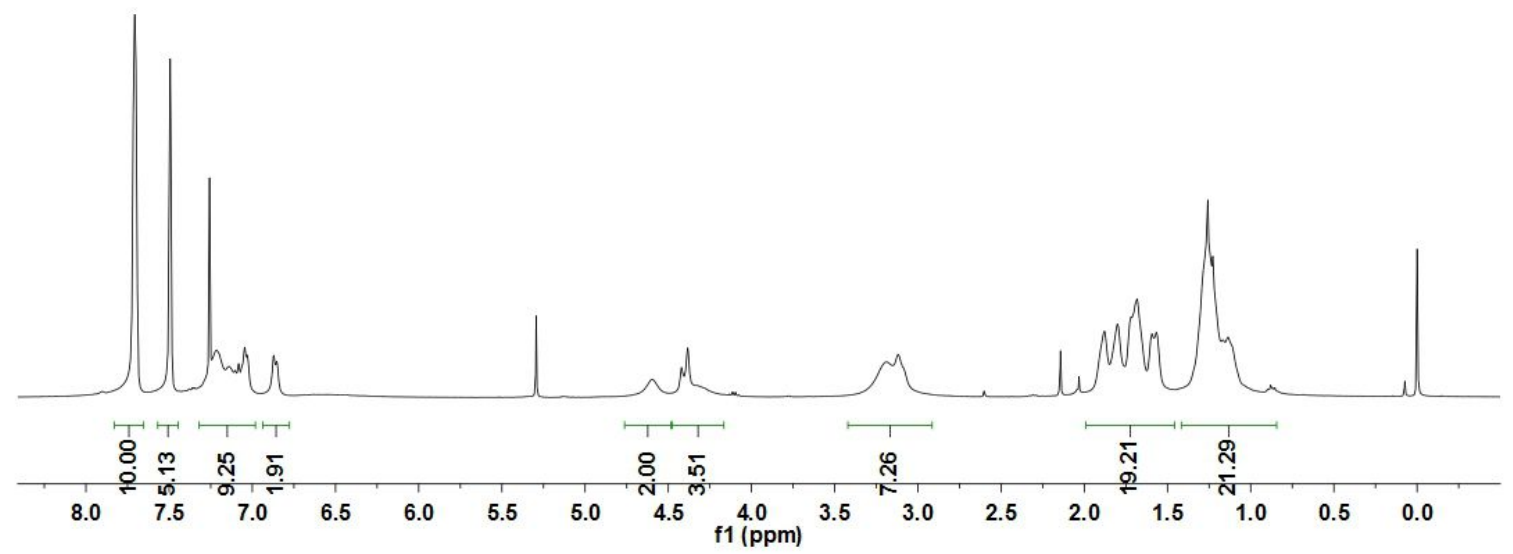

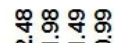

ஸัญ 흥

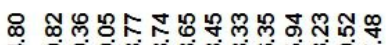

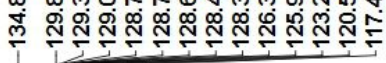

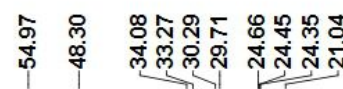

BG-3.1.3 HBAF $_{4}$

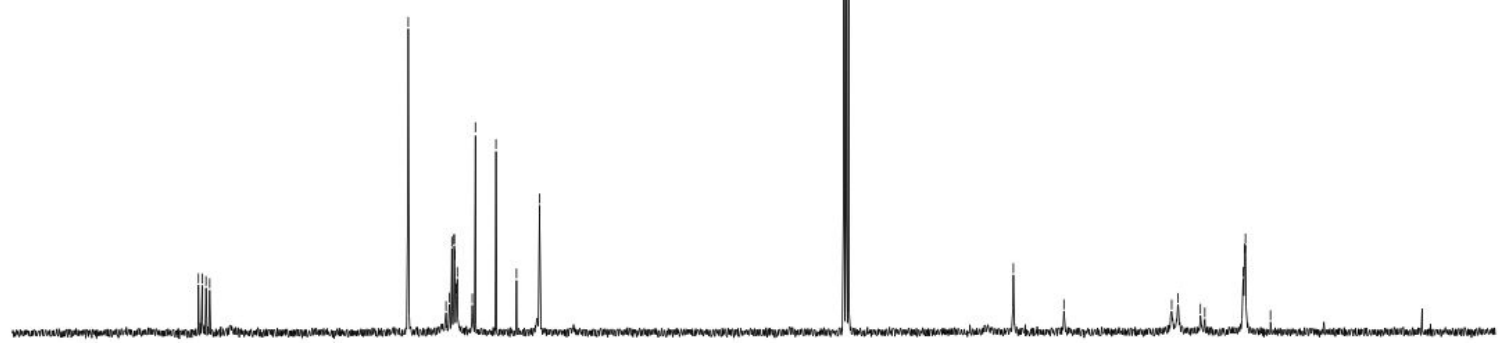

$\begin{array}{lllllllllllllllllll}180 & 170 & 160 & 150 & 140 & 130 & 120 & 110 & 100 & \underset{\mathrm{f} 1(\mathrm{ppm})}{90} 80 & 70 & 60 & 50 & 40 & 30 & 20 & 10 & 0\end{array}$ 
10. Copies of the CD spectra of the products 3

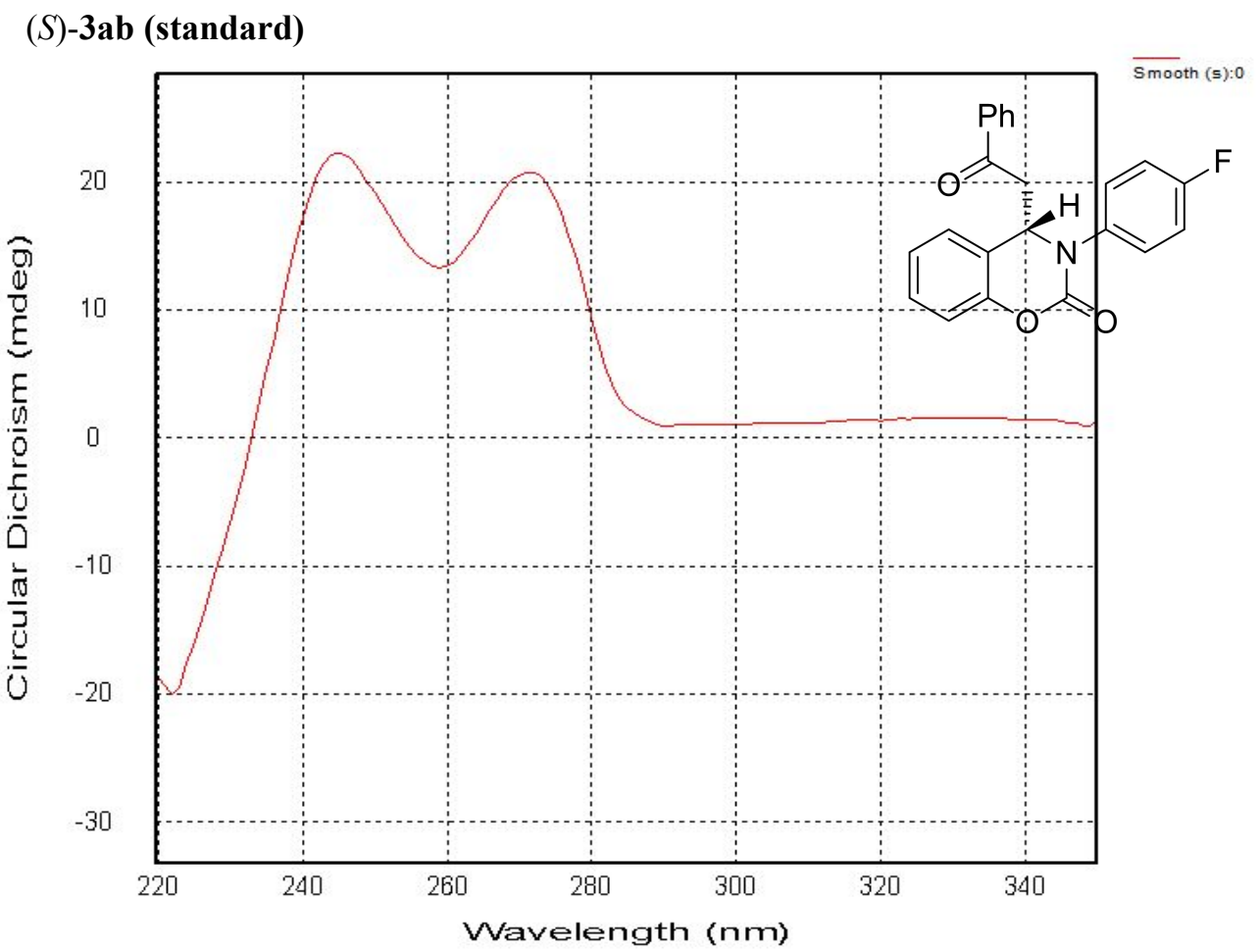

$3 \mathbf{b b}$

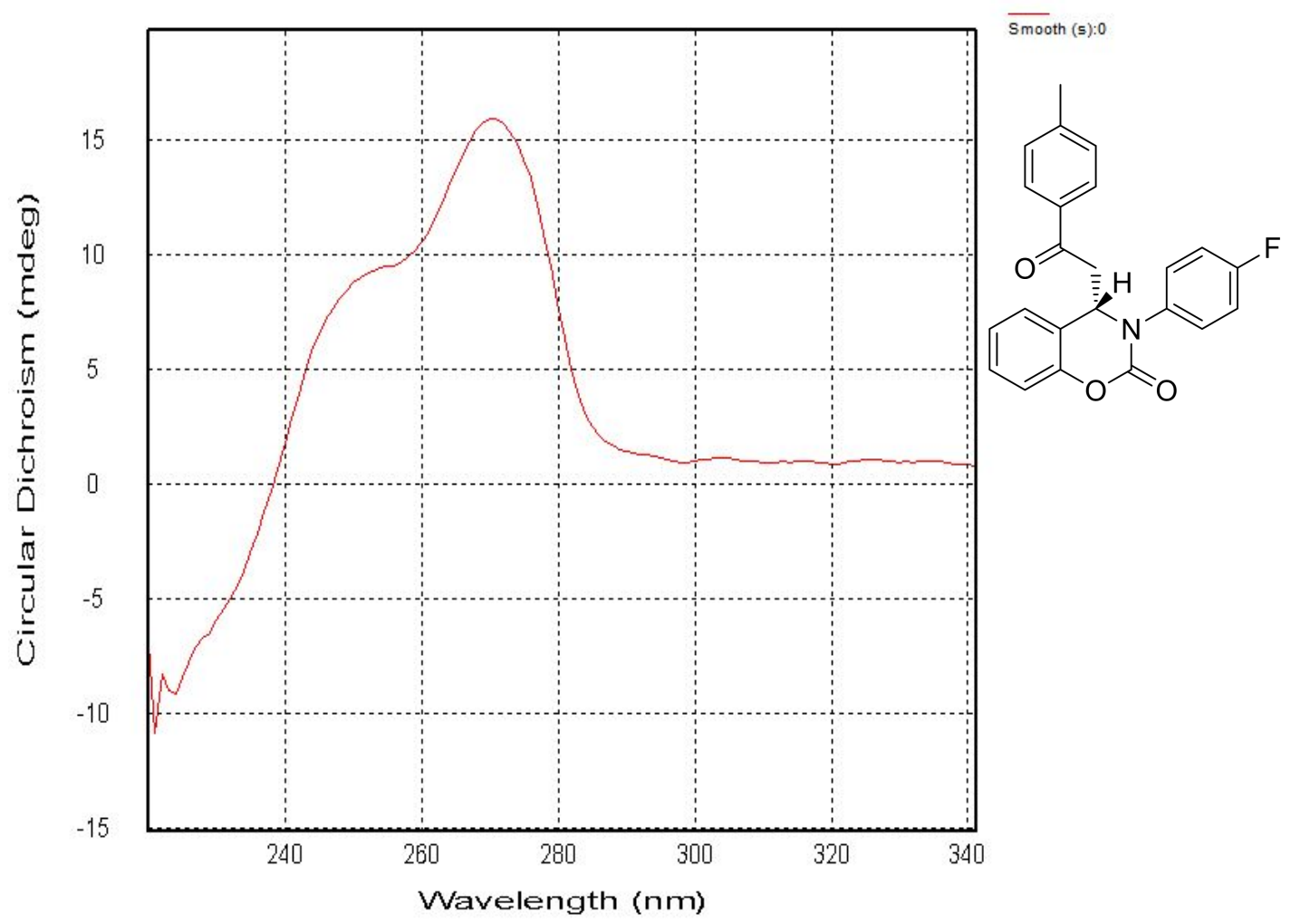




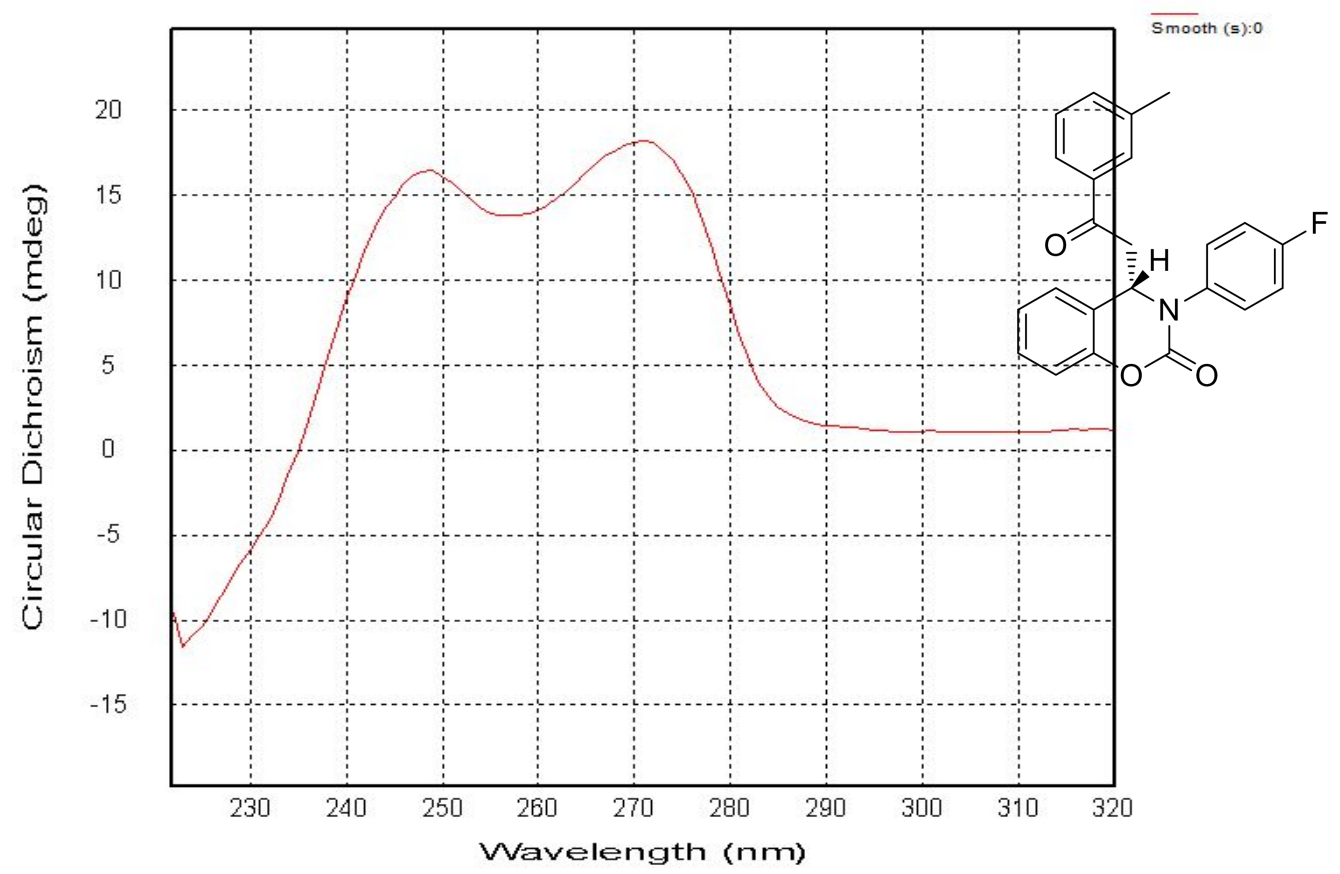

3db

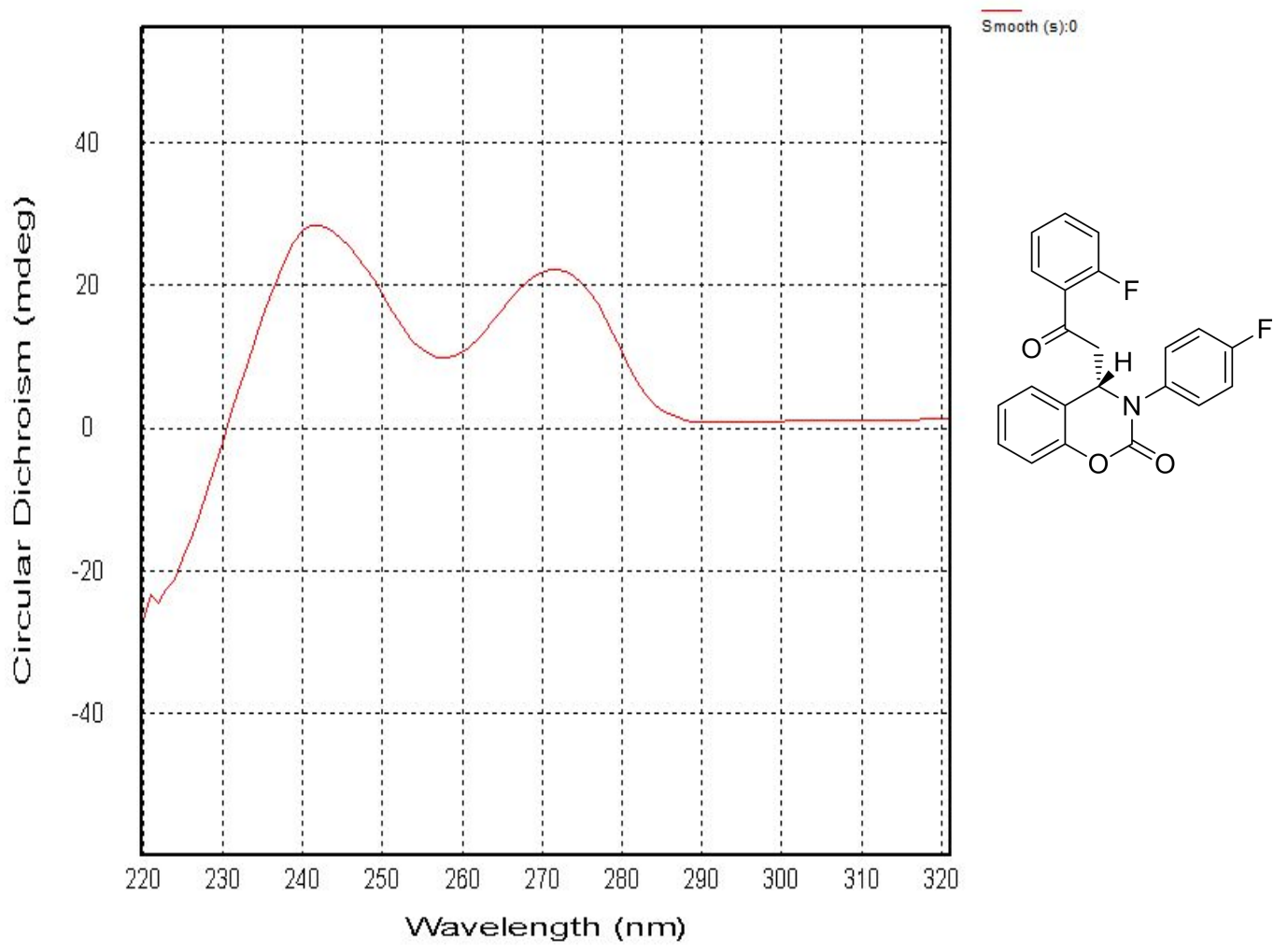




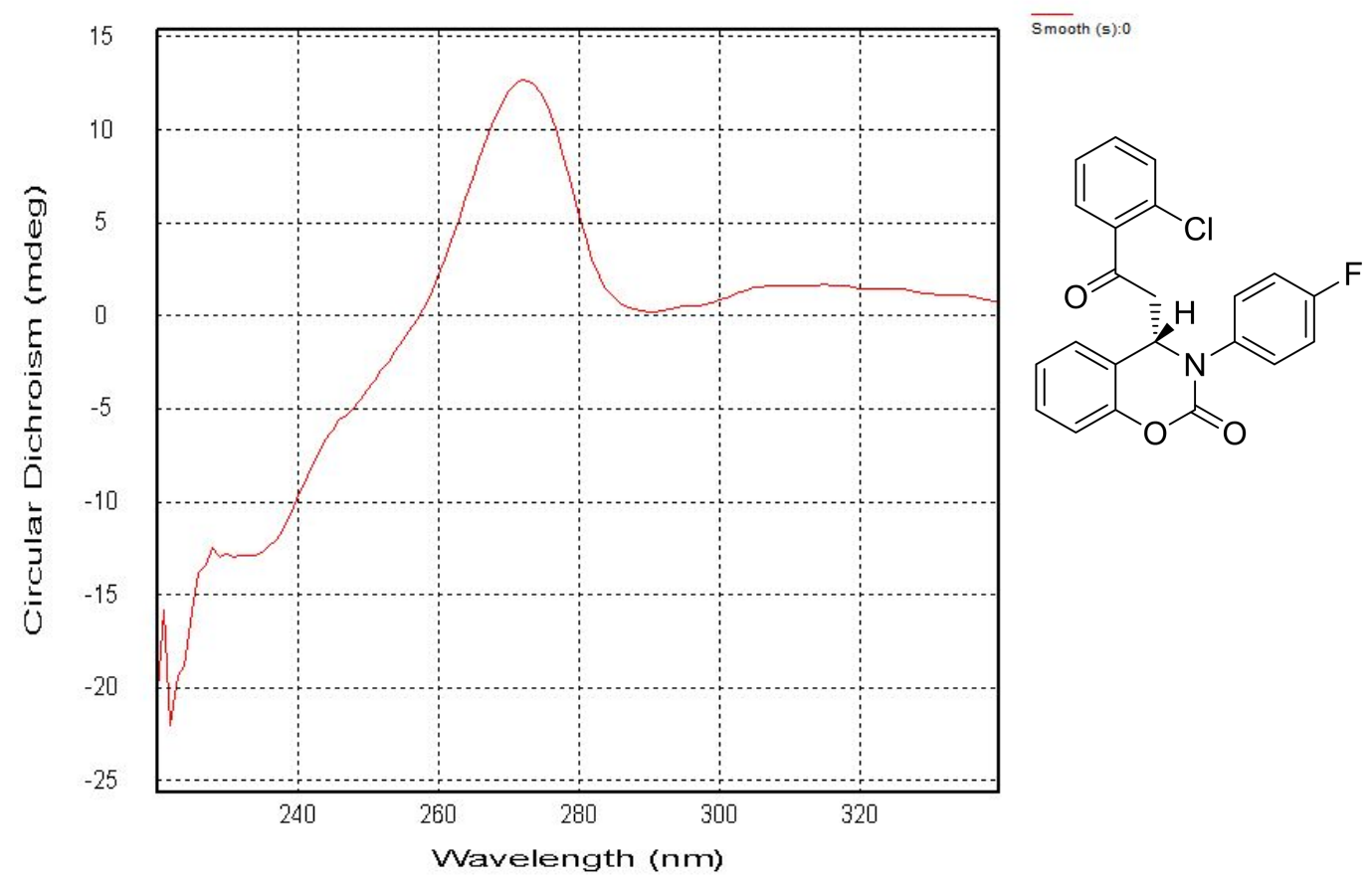

$3 f b$

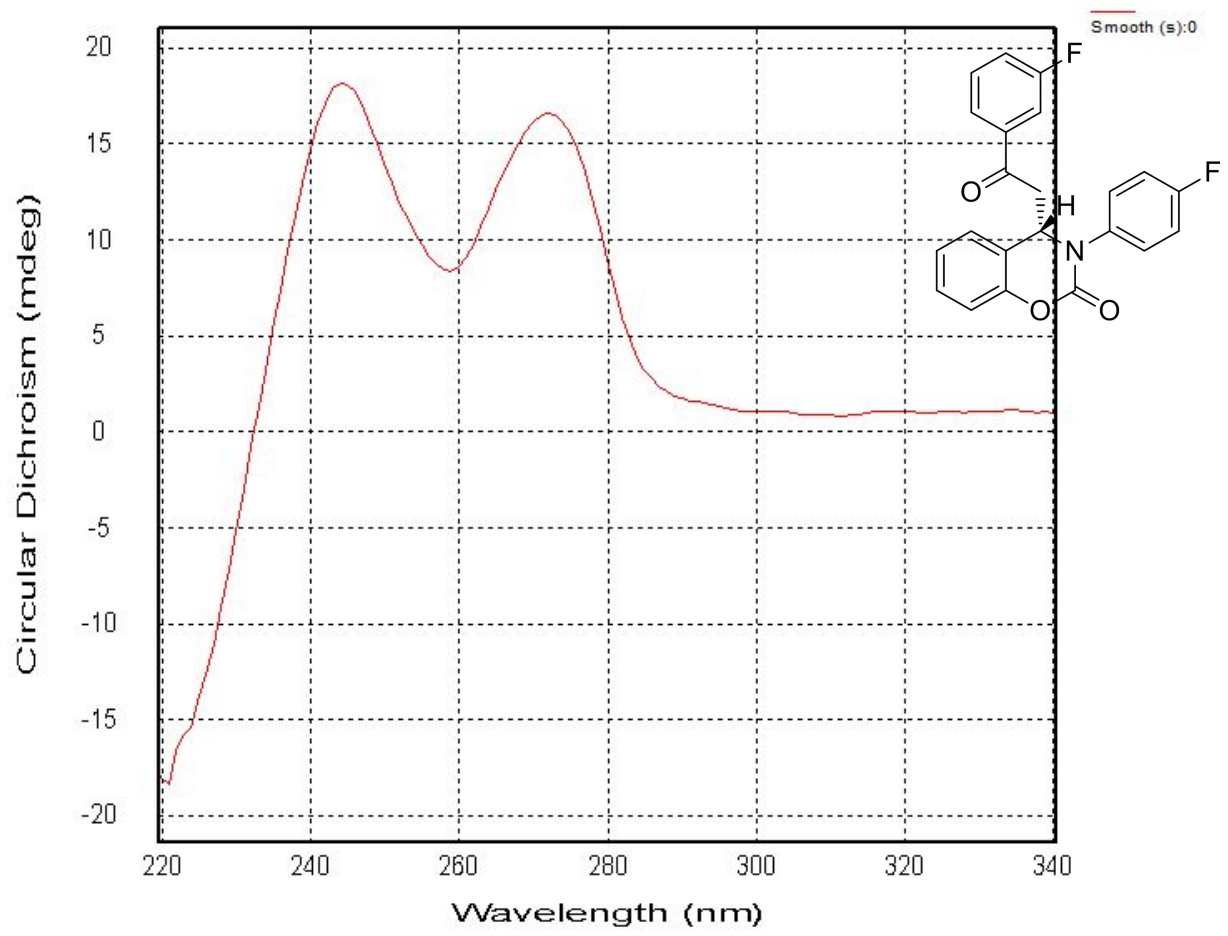


$3 g b$

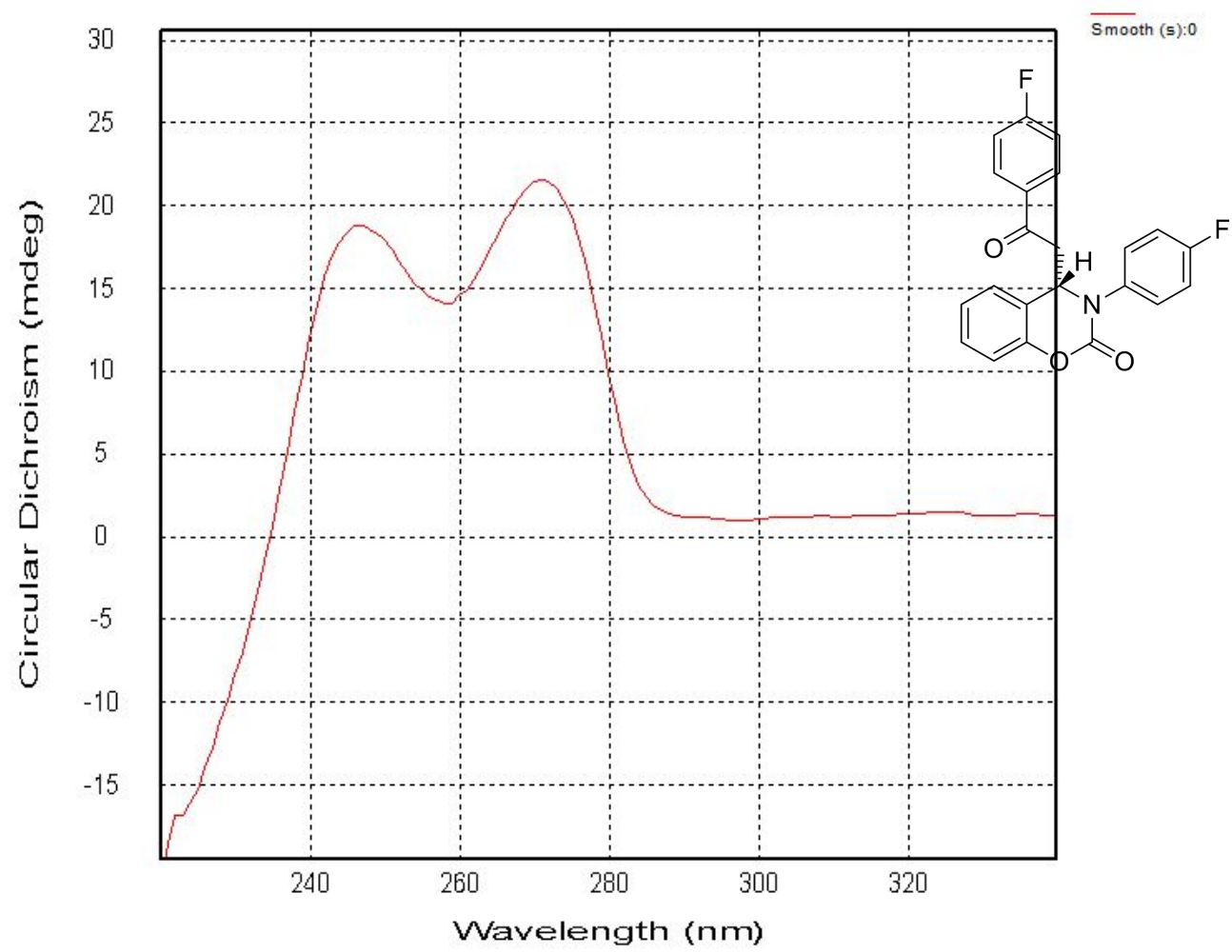

3hb

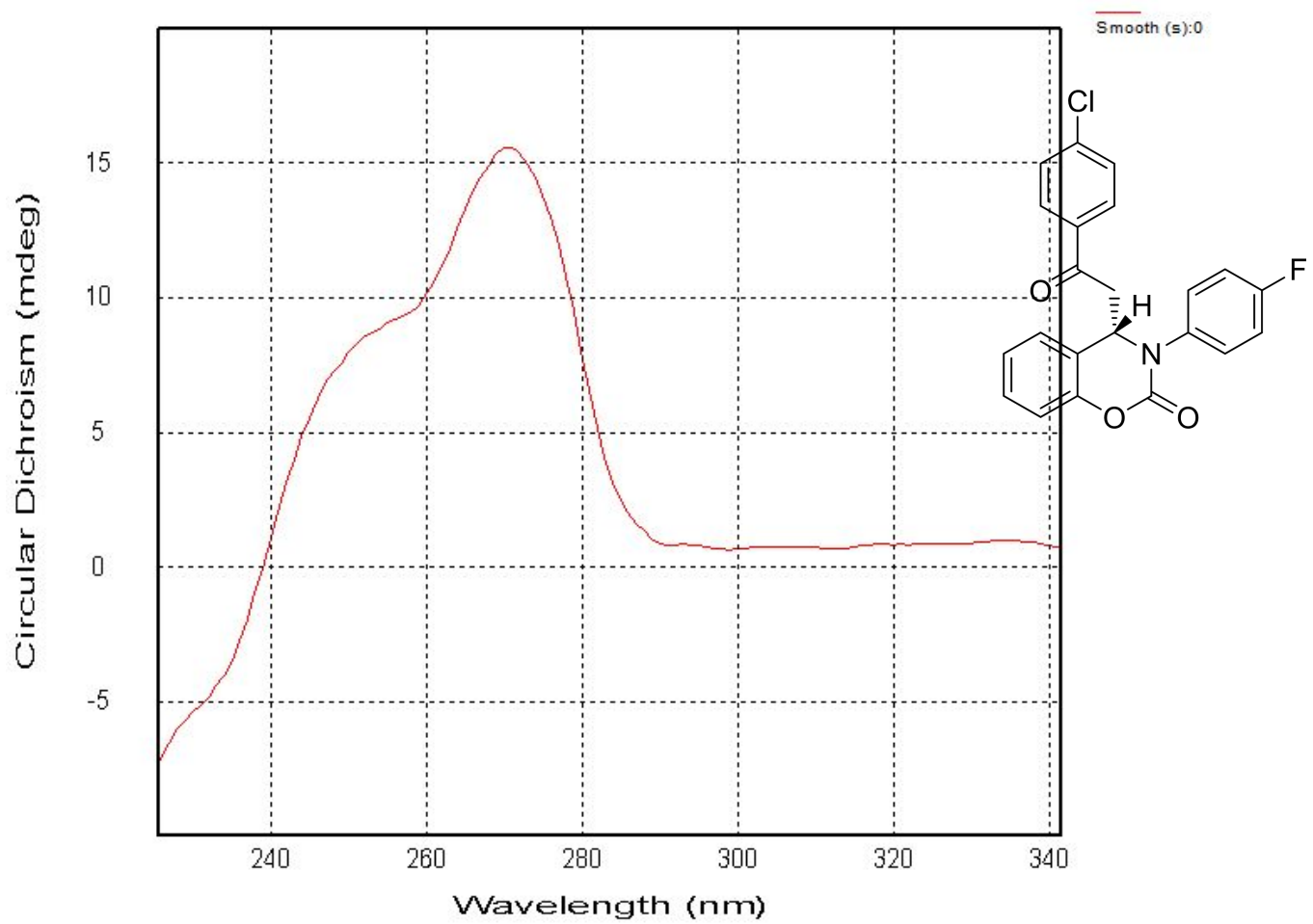




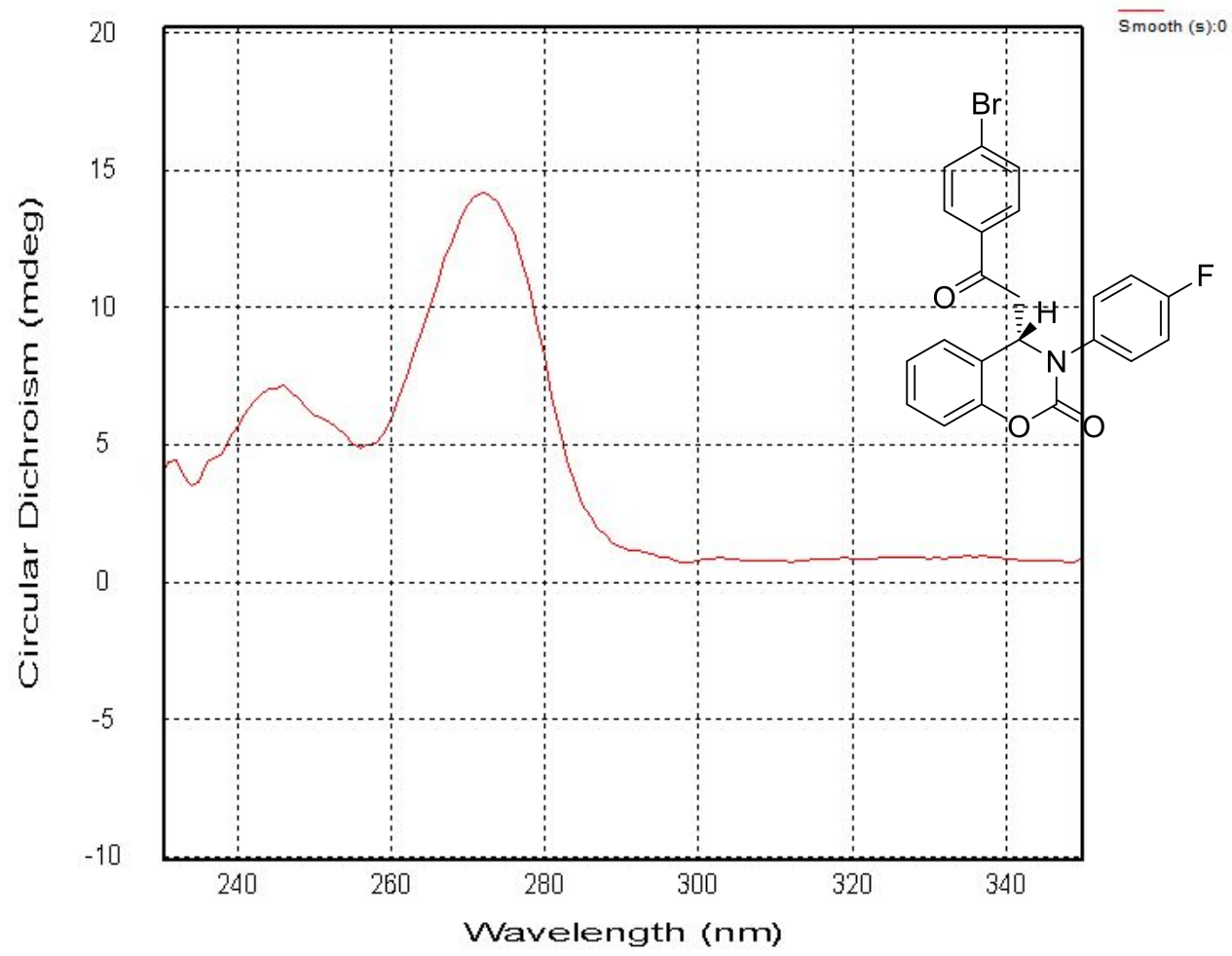

3jb

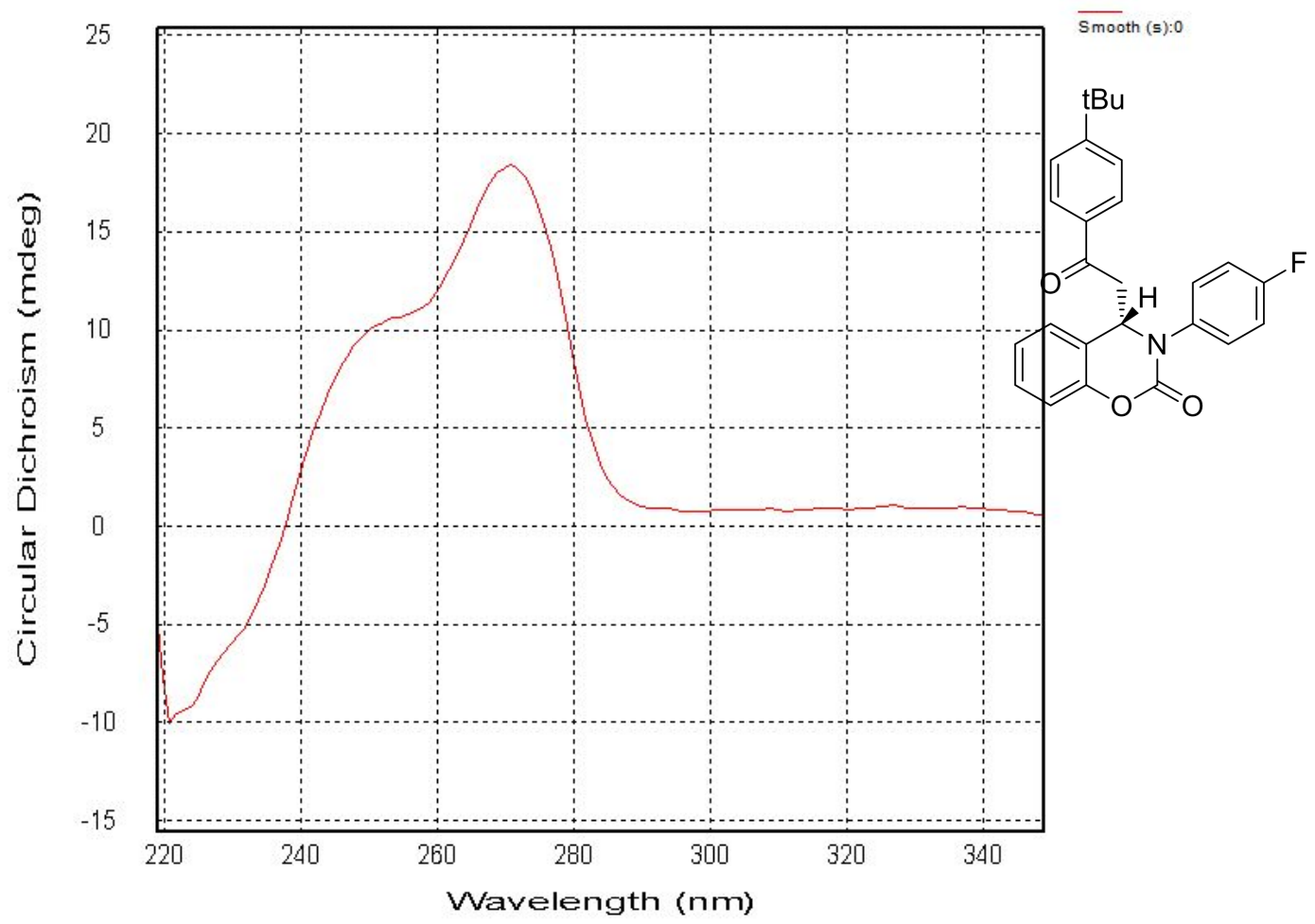




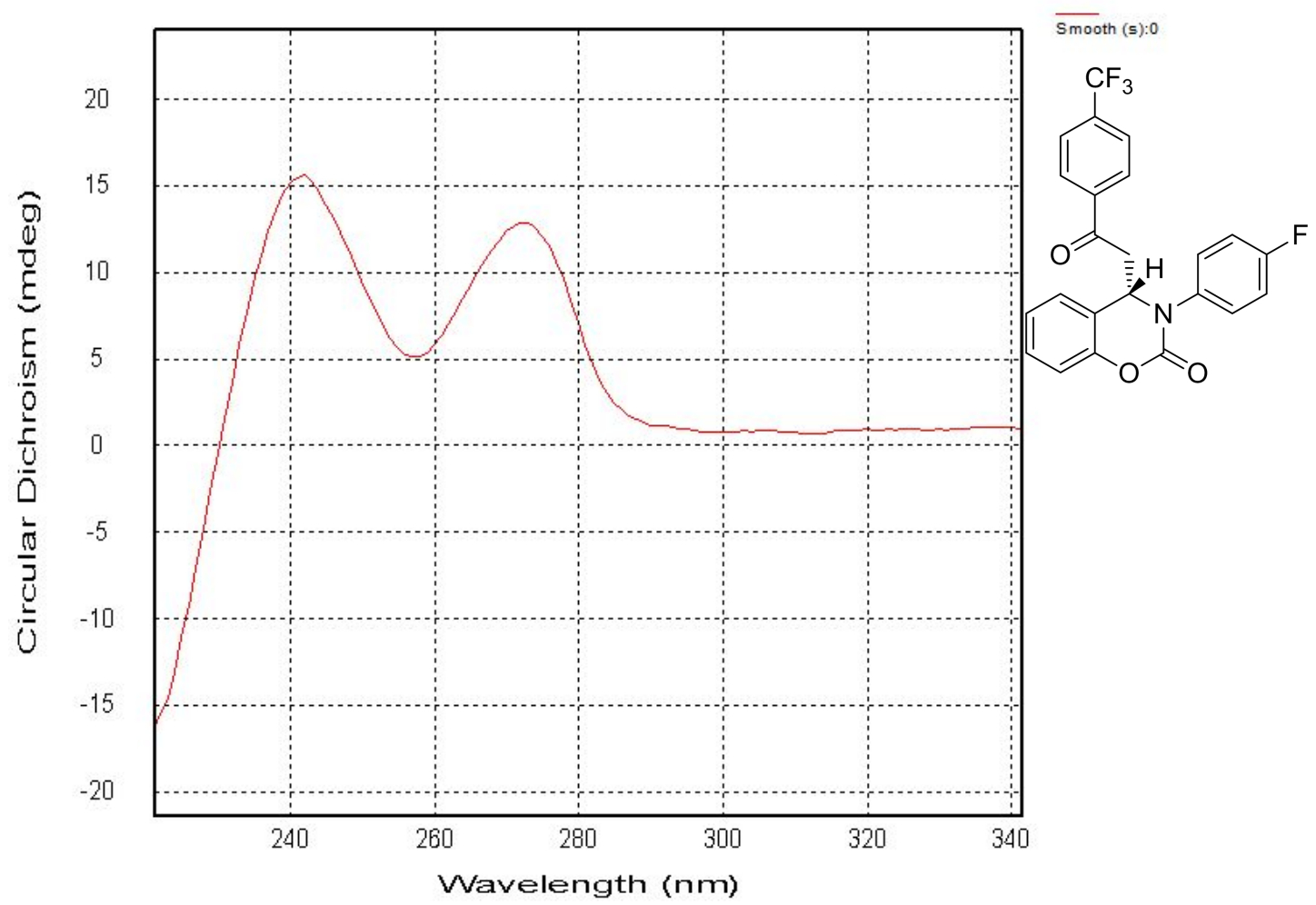

3lb

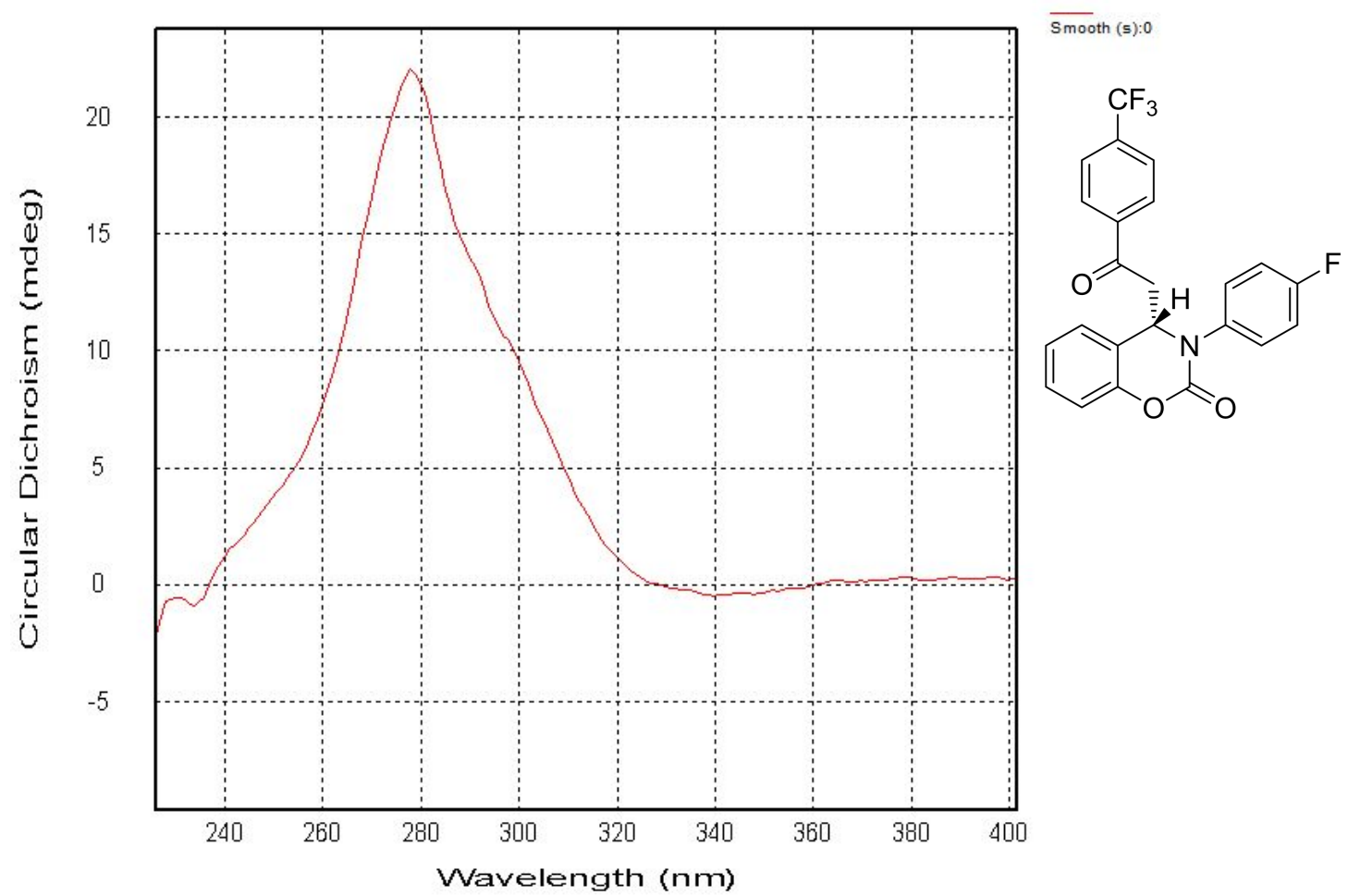


3mb

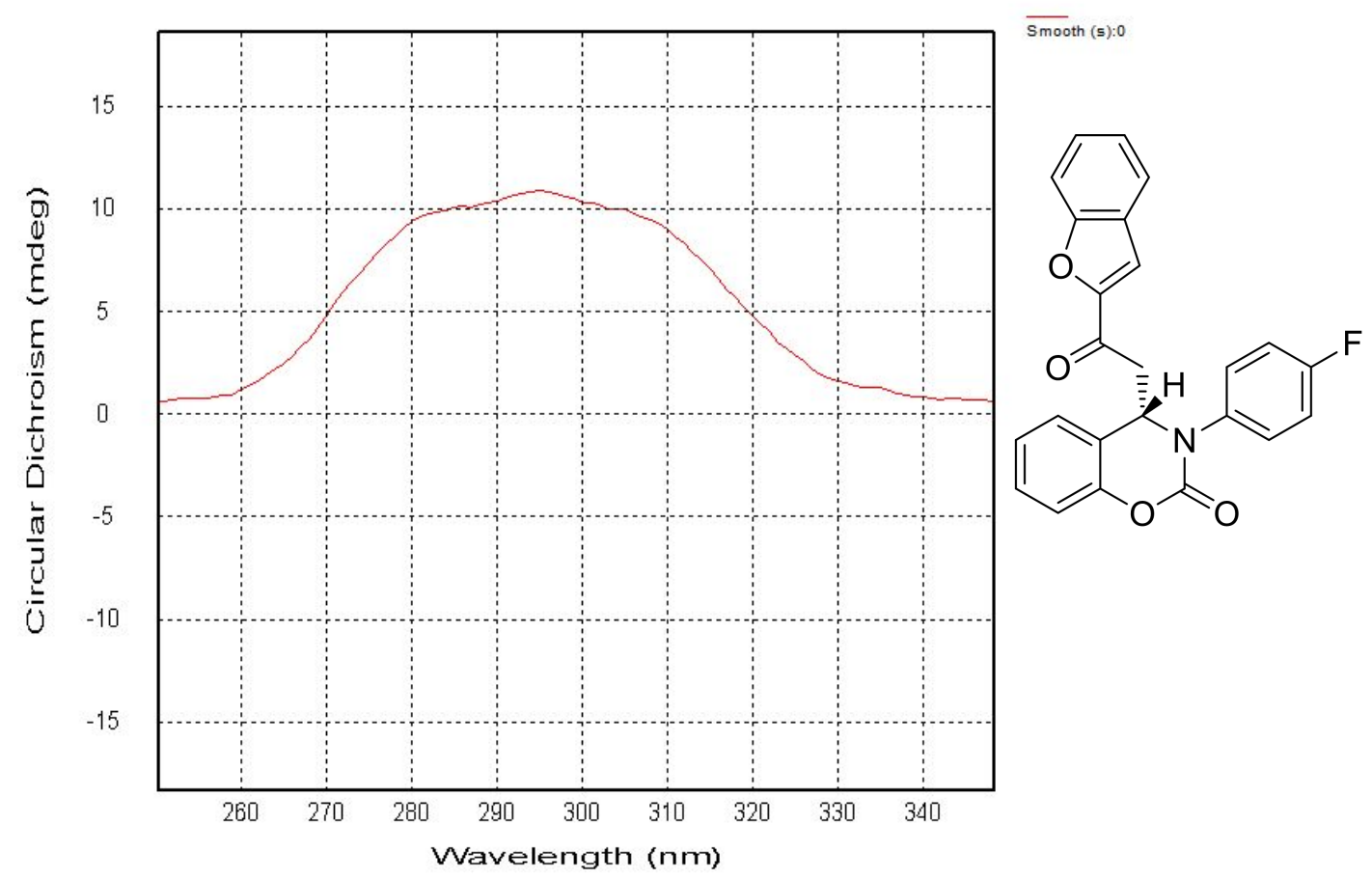

3nb

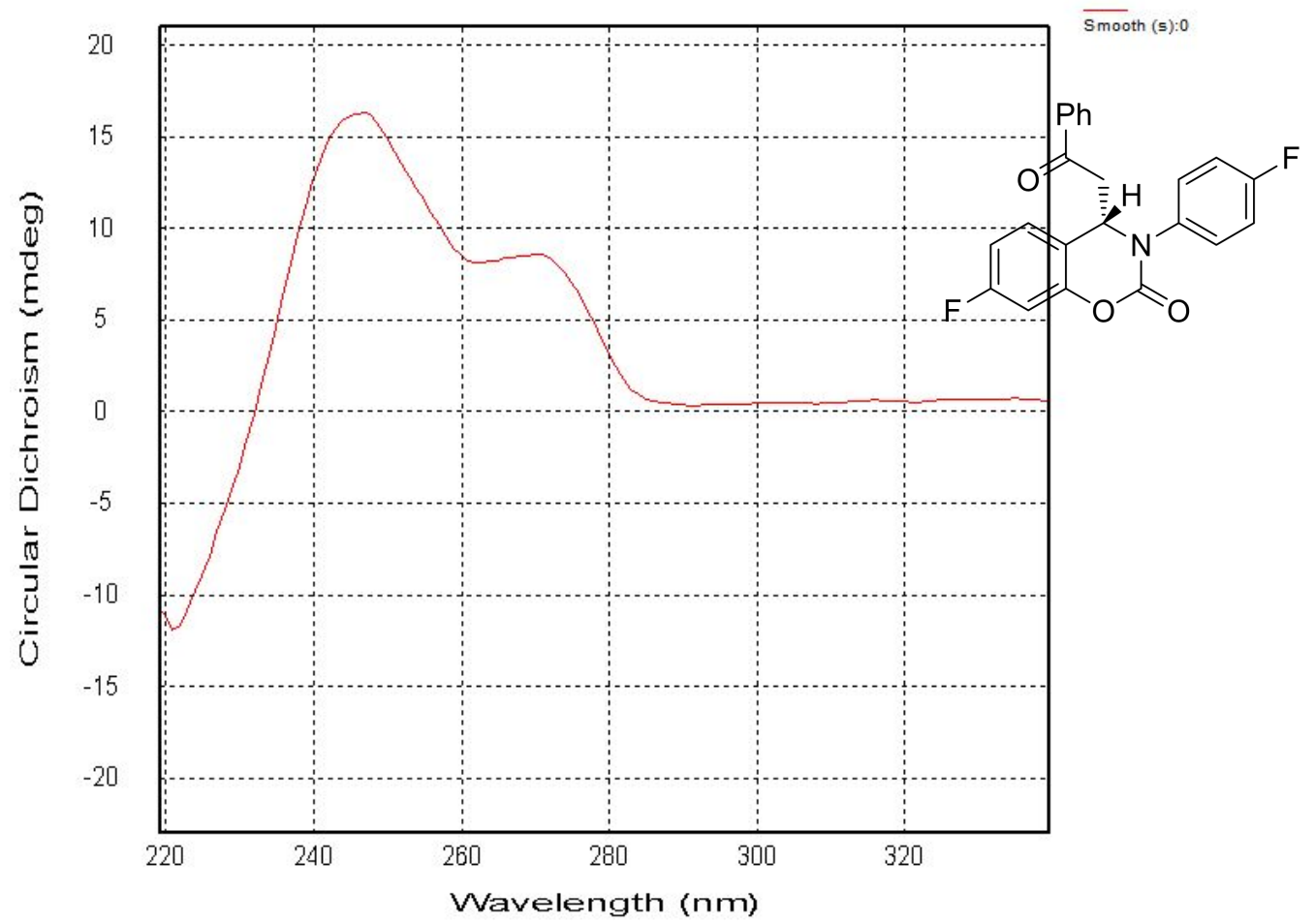




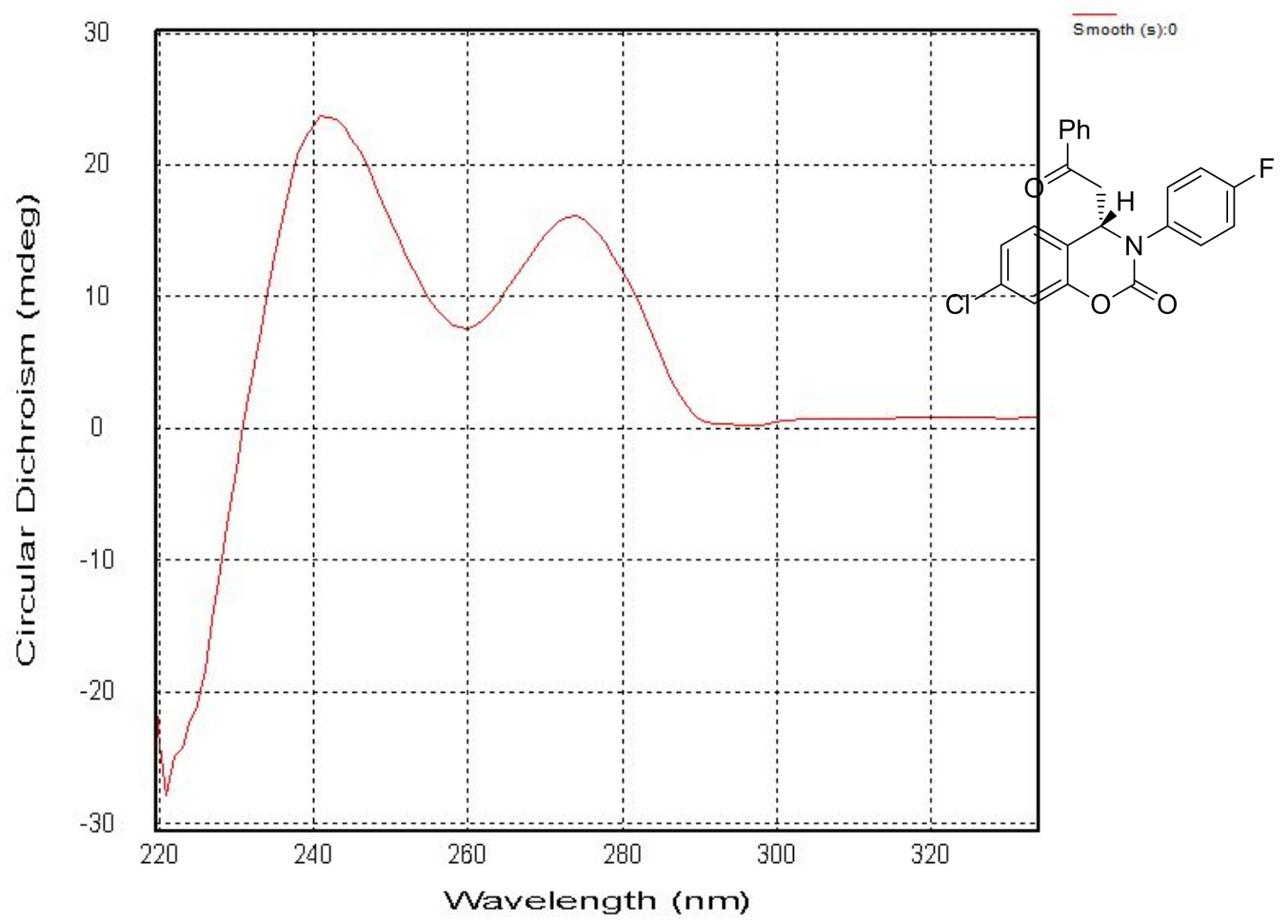

3pb

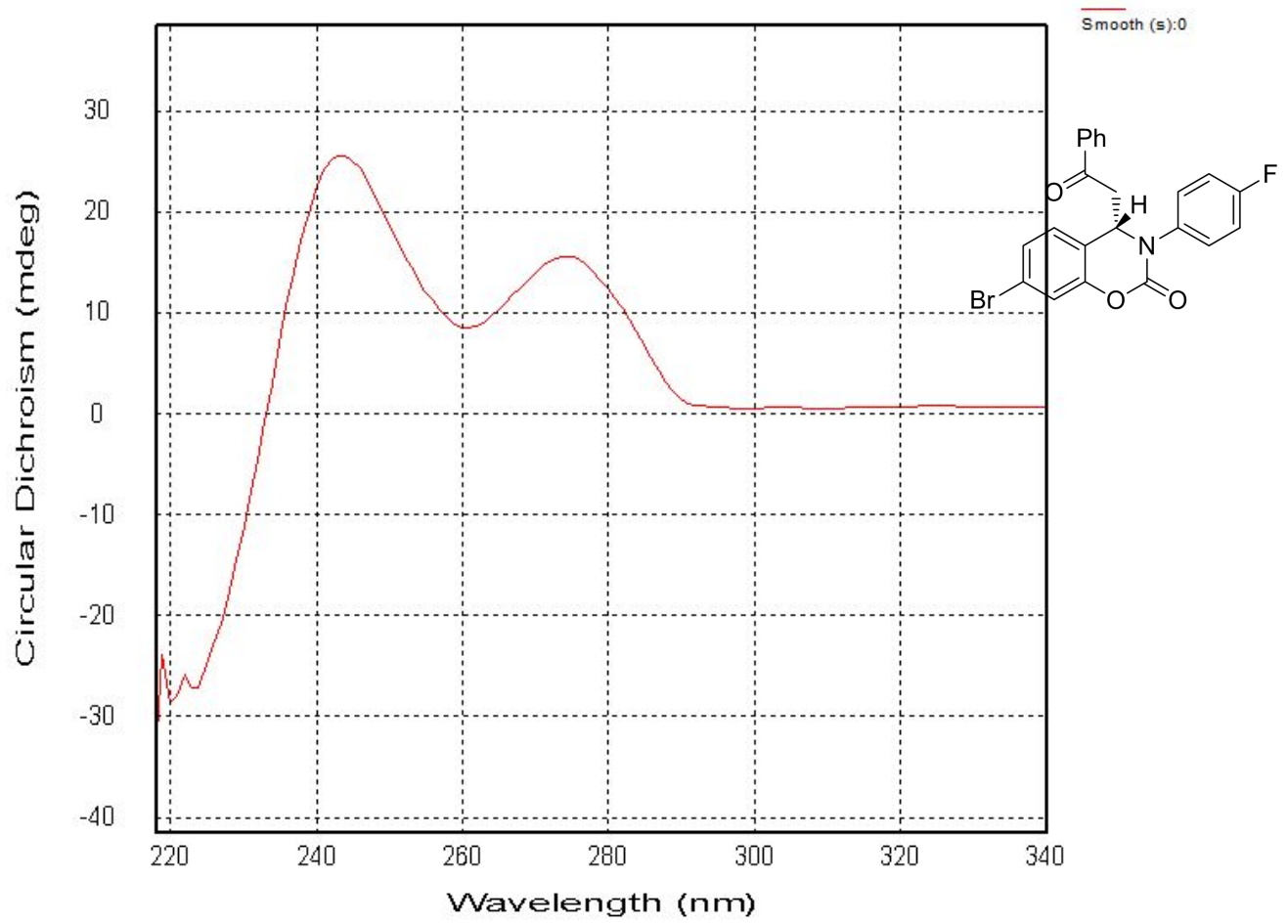




\section{$3 q b$}

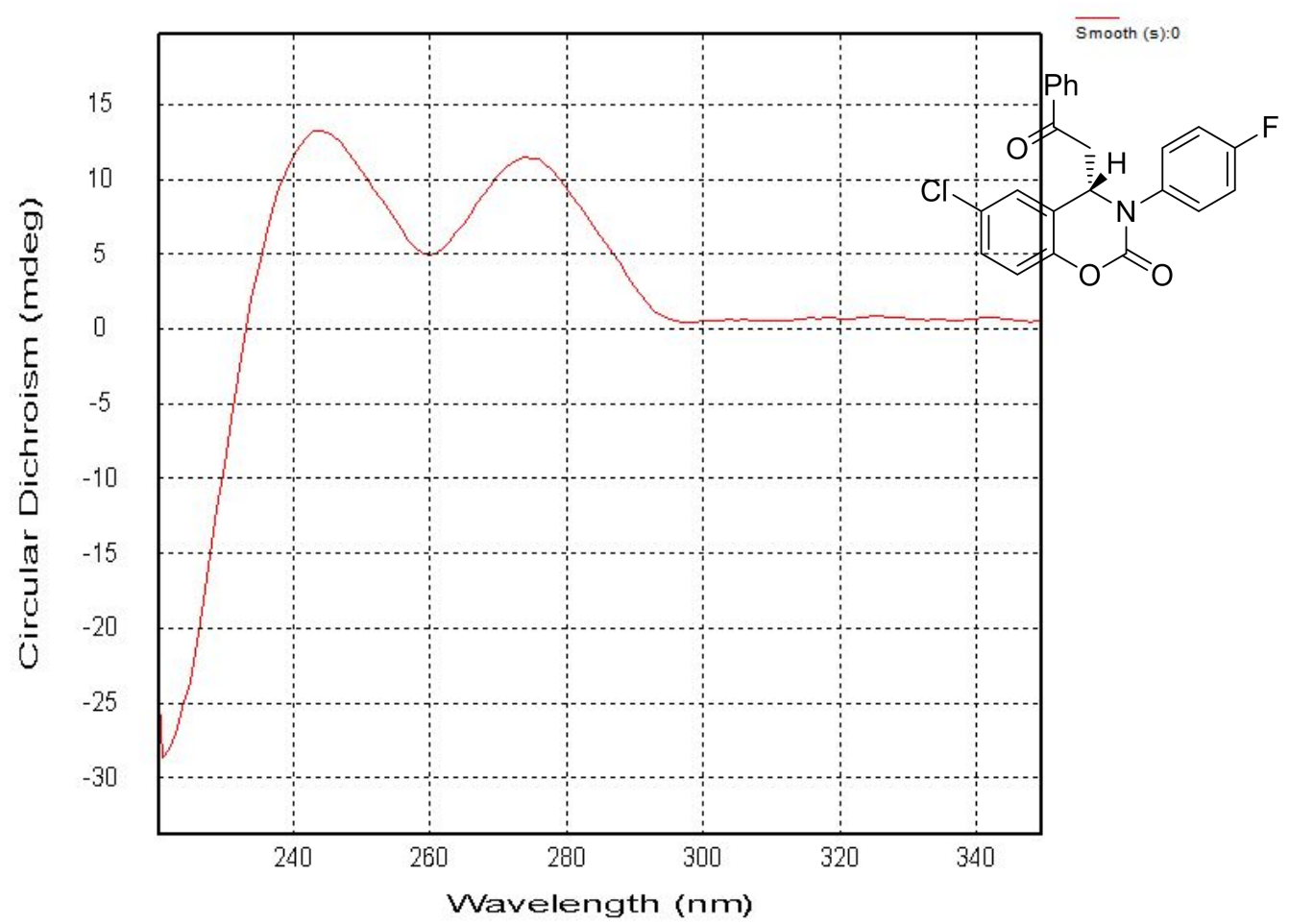

3rb

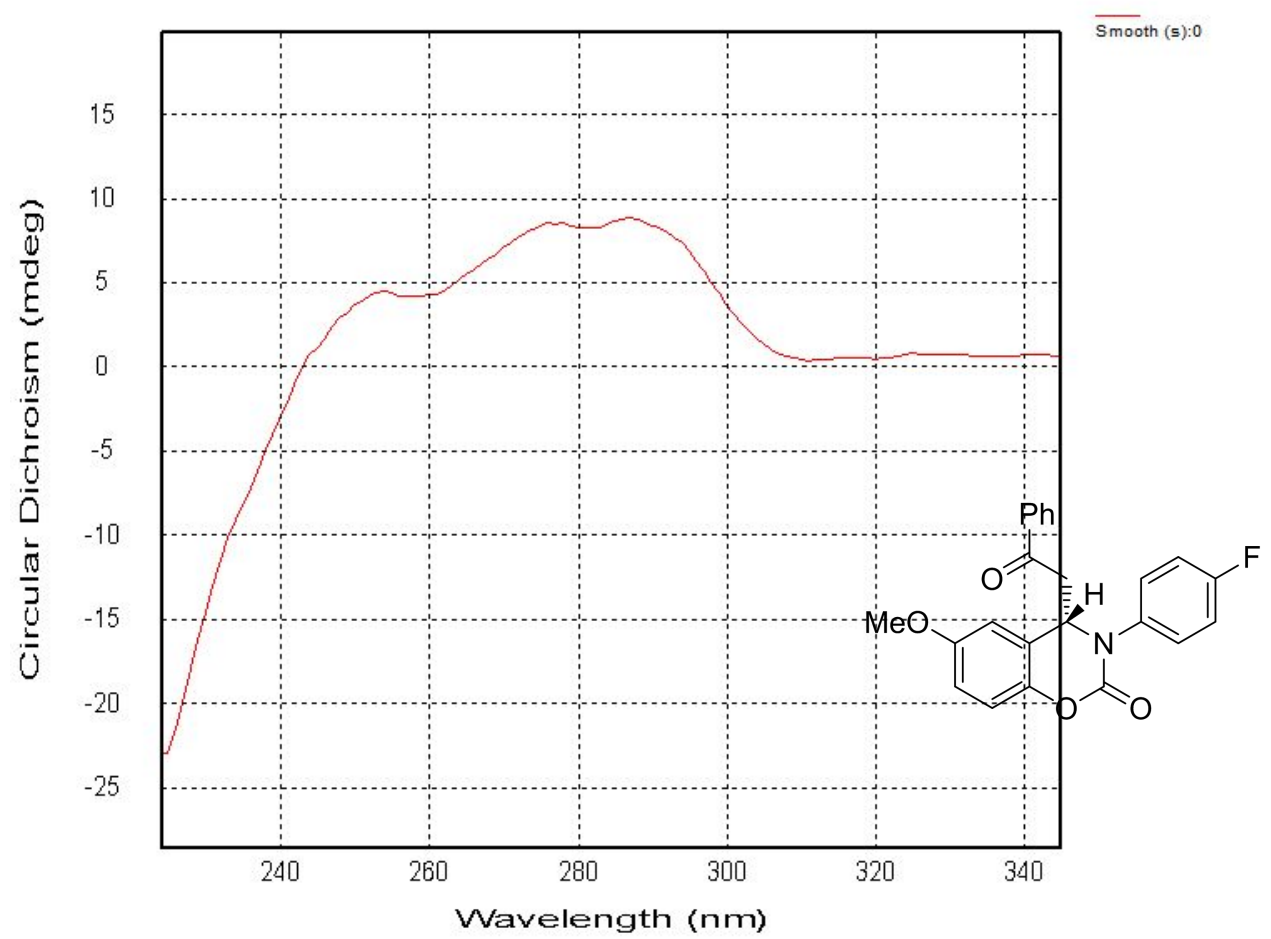


3sa

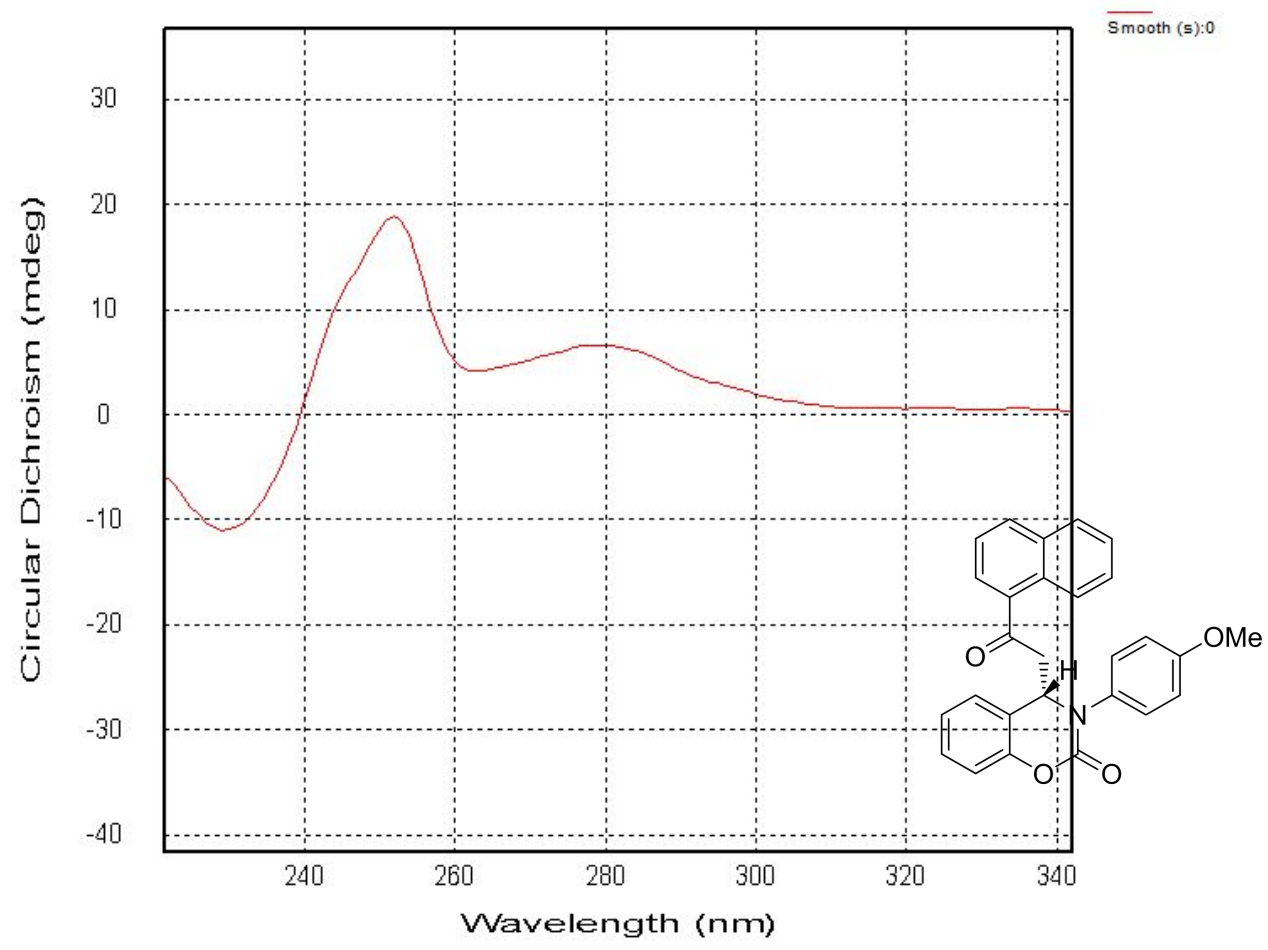

3 ta

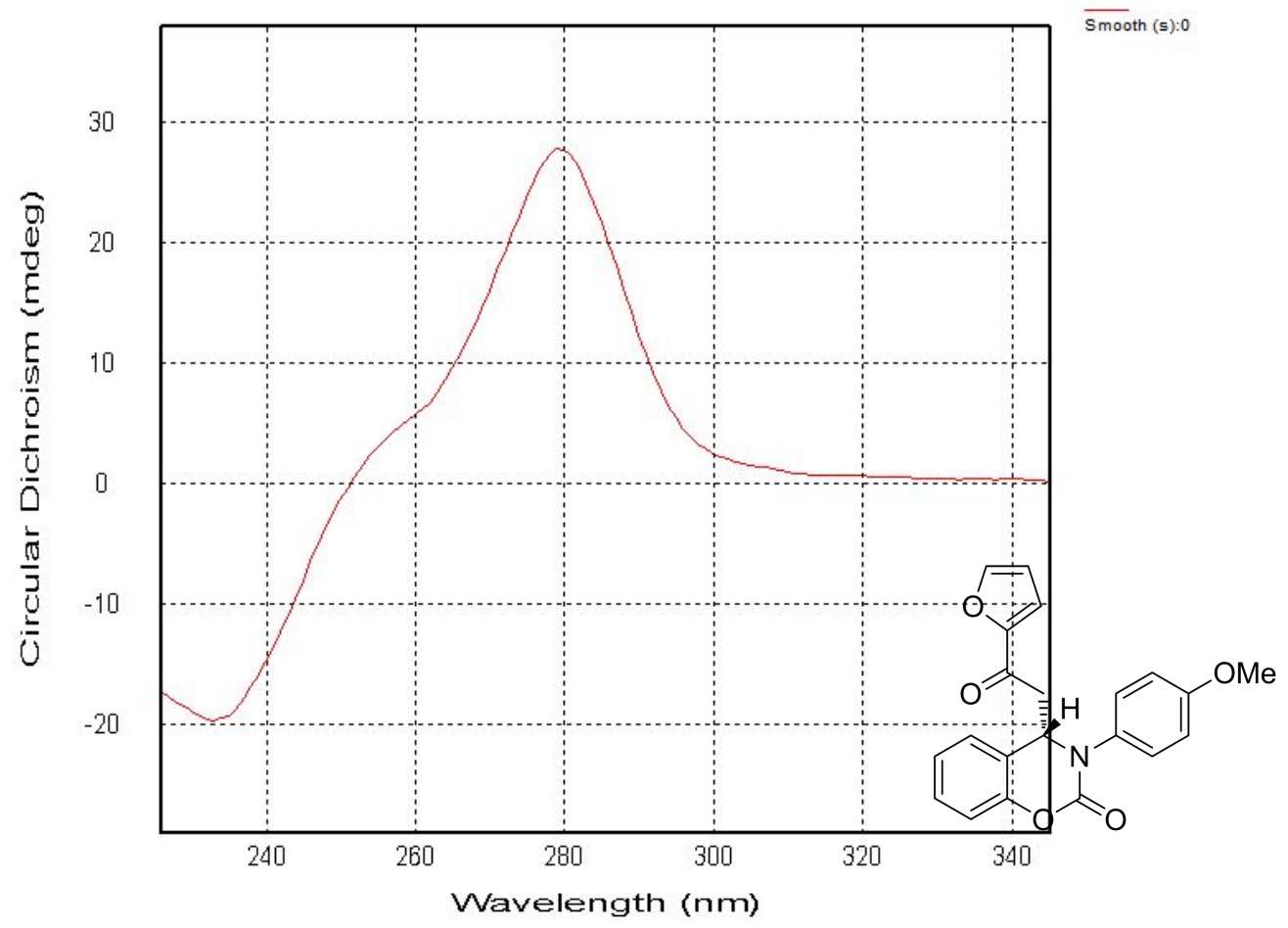




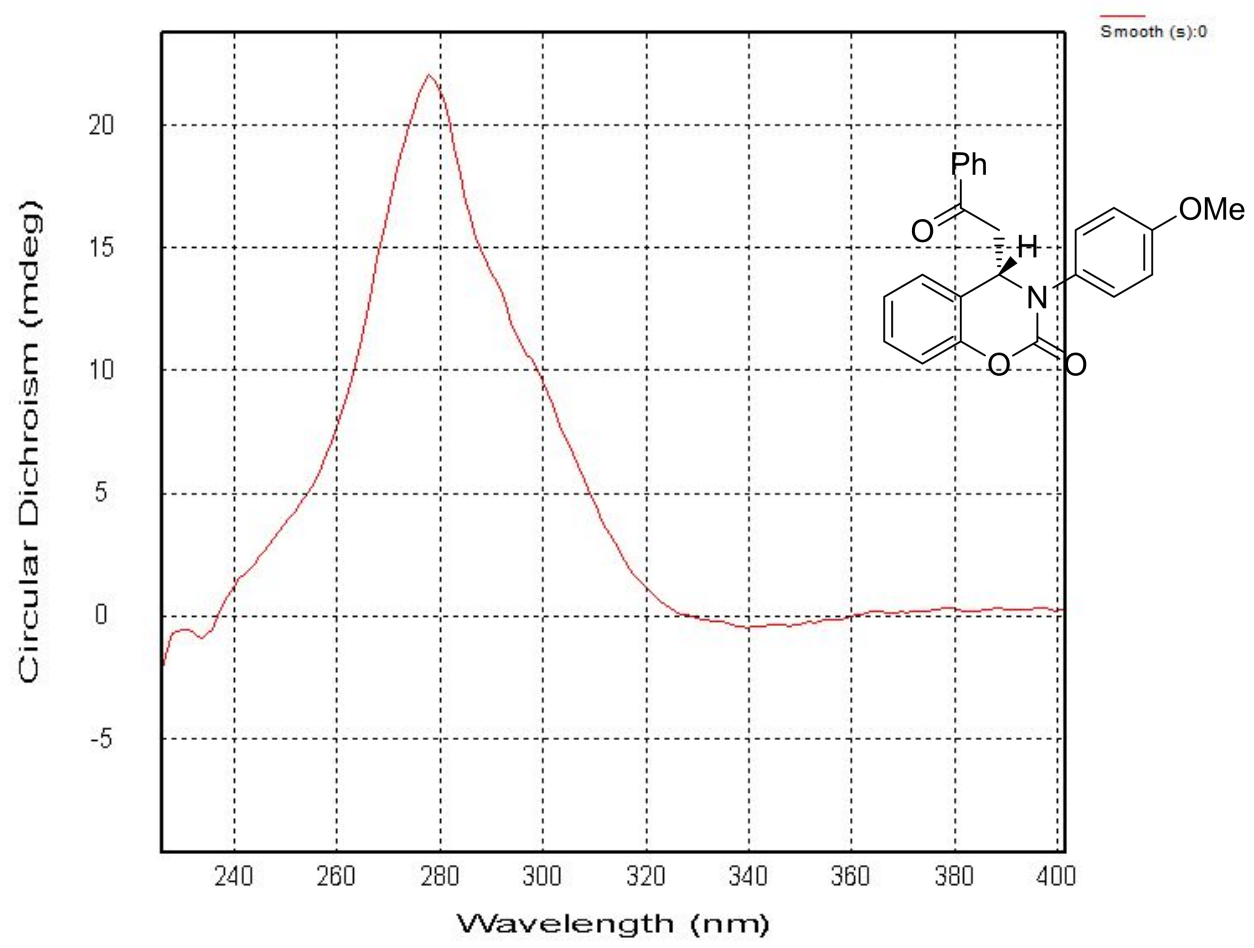

$3 a c$

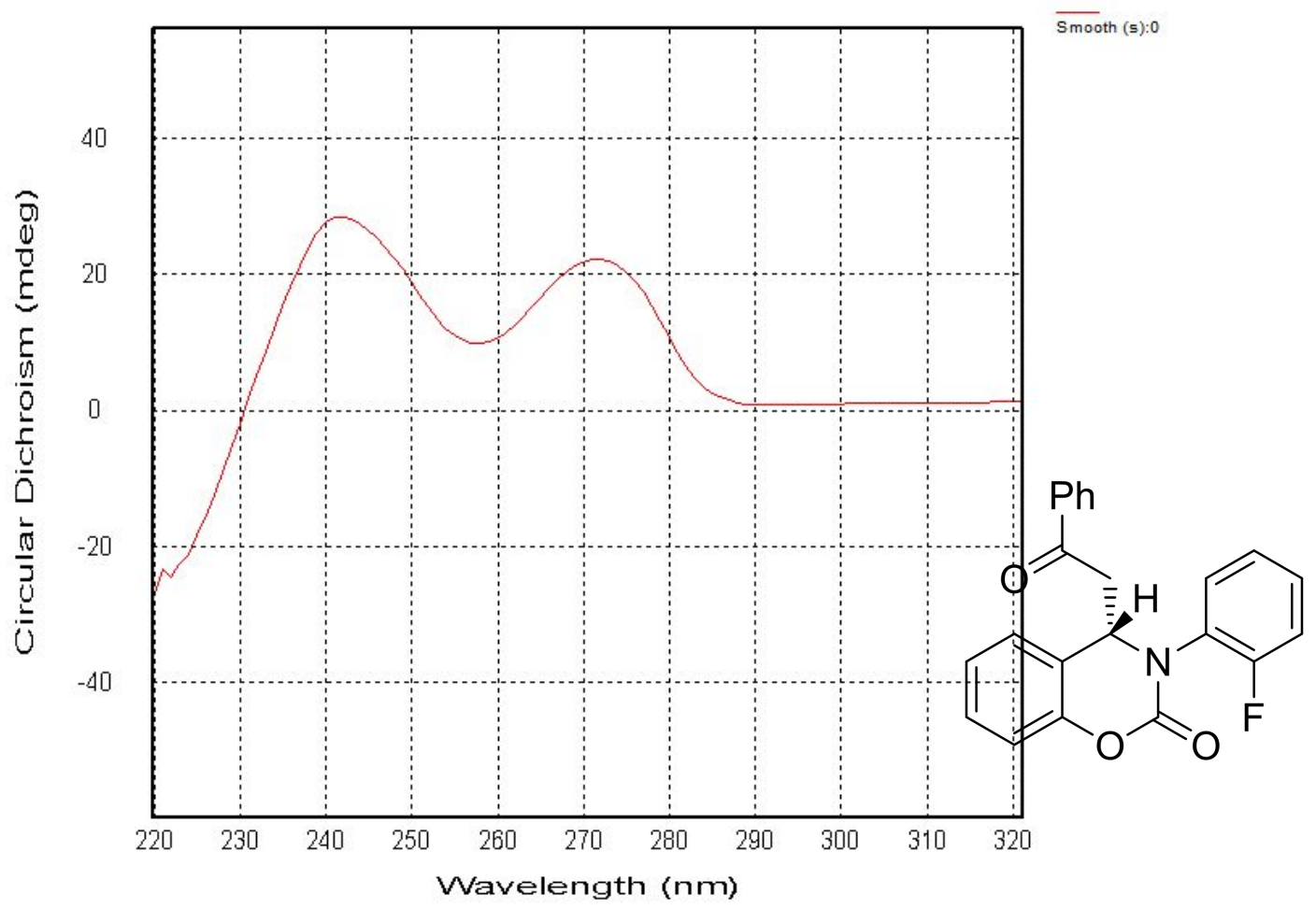




\section{$\mathrm{CD}$ spectra of the catalyst with variable ratio of $\mathrm{BG} / \mathrm{HBAr}_{4}{ }_{4}$}

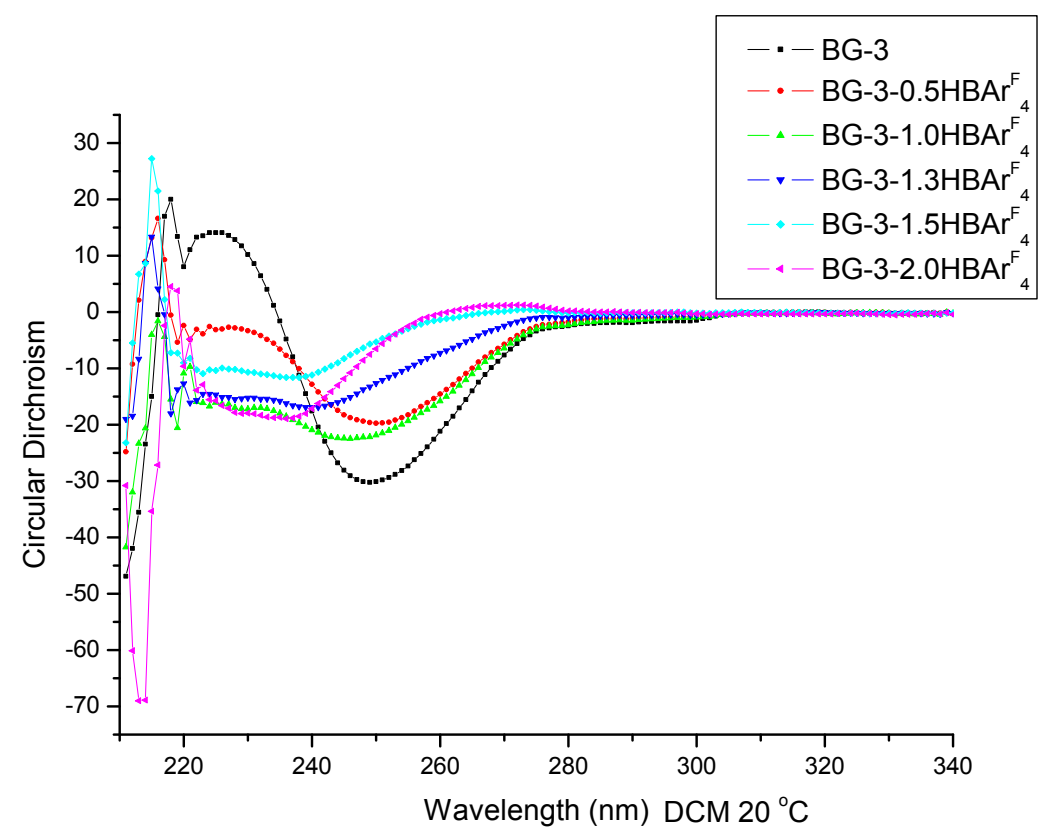

\section{References}

(1) (a) Mazimba, O.; Masesane, I. B.; Majinda, R. R. Tetrahedron Letters 2011, 52, 6716-6718. (b) Yin, G. D.; Fan, L.; Ren, T. B.; Zheng, C.Y.; Tao, Q.; Wu, A. X.; She, N. F. Org. Biomol. Chem. 2012, 10, 8877-8883.

(2) (a) Yu, Z. P.; Liu, X. H.; Zhou, L.; Lin, L. L.; Feng, X. M. Angew.Chem. Int. Ed. 2009, 48, 5195-5198. (b) Dong, S. X.; Liu, X. H.; Chen, X. H.; Mei, F,; Zhang, Y. L; Lin, L. L.; Feng, X. M. J. Am. Chem. Soc. 2010, 132, 10650-10651.

(3) (a) Uyeda, C.; Jacobsen, E. N. J. Am. Chem. Soc. 2008, 130, 9228-9229. (b) Fu, X.; Loh, W. T.; Zhang, Y.; Chen, T.; Ma, T.; Liu, H. J.; Wang, J. M.; Tan, C. H. Angew. Chem., Int. Ed. 2009, 48, 7387-7390. 